\title{
1 Root hydrotropism is controlled via a cortex-specific growth mechanism
}

3 Daniela Dietrich ${ }^{1,2, \S}$, Lei Pang ${ }^{3, \S}$, Akie Kobayashis ${ }^{3 \S}$, John A. Fozard ${ }^{1, \dagger}$, Véronique Boudolf ${ }^{4,5}$, 4 Rahul Bhosale ${ }^{1,2,4,5}$, Regina Antoni ${ }^{1, \dagger}$, Tuan Nguyen ${ }^{1,6}$, Sotaro Hiratsuka ${ }^{3}$, Nobuharu Fujii ${ }^{3}$, 5 Yutaka Miyazawa7, Tae-Woong Bae ${ }^{3}$, Darren M. Wells ${ }^{1,2}$, Markus R. Owen ${ }^{1,8}$, Leah R. Band ${ }^{1,8}$, 6 Rosemary J. Dyson ${ }^{9}$, Oliver E. Jensen ${ }^{1,10}$, John R. King ${ }^{1,8}$, Saoirse R. Tracy ${ }^{1,11, \dagger}$, Craig J. 7 Sturrock ${ }^{1,11}$, Sacha J. Mooney ${ }^{1,11}$, Jeremy A. Roberts ${ }^{1,2 \dagger}$, Rishikesh P. Bhalerao ${ }^{12,13}$, José R. Dinneny ${ }^{14}$, Pedro L. Rodriguez ${ }^{15}$, Akira Nagatani ${ }^{16}$, Yoichiroh Hosokawa ${ }^{17}$, Tobias I. Baskin ${ }^{1,18}$, Tony P. Pridmore ${ }^{1,6}$, Lieven De Veylder ${ }^{4,5}$, Hideyuki Takahashi ${ }^{\sharp *}$ and Malcolm J. Bennett ${ }^{1,2 \sharp^{*}}$

${ }^{1}$ Centre for Plant Integrative Biology, University of Nottingham, Nottingham, LE12 5RD, UK 2Plant \& Crop Sciences, School of Biosciences, University of Nottingham, Nottingham, LE12 5RD, UK ${ }^{3}$ Graduate School of Life Sciences, Tohoku University, Sendai 980-8577, Japan ${ }^{4}$ Ghent University, Department of Plant Biotechnology and Bioinformatics, (Technologiepark 927), 9052 Ghent, Belgium

${ }^{5}$ VIB Center for Plant Systems Biology, (Technologiepark 927), 9052 Ghent, Belgium ${ }^{6}$ School of Computer Science, University of Nottingham, Nottingham, NG8 1BB, UK ${ }^{7}$ Faculty of Science, Yamagata University, Yamagata 990-8560, Japan

${ }^{8}$ Centre for Mathematical Medicine \& Biology, University of Nottingham, Nottingham, NG7 2RD, UK ${ }^{9}$ School of Mathematics, University of Birmingham, Birmingham, B15 2TT, UK

${ }^{10}$ School of Mathematics, University of Manchester, Oxford Road, Manchester, M13 9PL, UK

${ }^{11}$ Agricultural and Environmental Sciences, School of Biosciences, University of Nottingham, Nottingham, LE12 5RD, UK

${ }^{12}$ Department of Forest Genetics and Plant Physiology, SLU, S-901 83 Umea, Sweden

${ }^{13}$ College of Science, KSU, Riyadh, Saudi Arabia

${ }^{14}$ Carnegie Institution for Science, Department of Plant Biology, 260 Panama Street, Stanford, CA 94305, USA

${ }^{15}$ Instituto de Biología Molecular y Celular de Plantas, Consejo Superior de Investigaciones Cientificas-Universidad Politecnica de Valencia, ES-46022 Valencia, Spain

${ }^{16}$ Graduate School of Science, Kyoto University, Kyoto 606-8502, Japan

${ }^{17}$ Graduate School of Materials Science, Nara Institute of Science \& Technology, Ikoma 630-0101, Japan

${ }^{18}$ Biology Department, University of Massachusetts, Amherst, Massachusetts, USA

† Present address: Computational and Systems Biology, John Innes Centre, Norwich NR4 7UH, UK (J.A.F.); Centre Nacional d'Anàlisi Genòmica (CNAG-CRG) 08028 Barcelona, Spain (R.A.); School of Agriculture and Food Science, University College Dublin, Belfield Campus, Dublin 4, Ireland (S.R.T.); School of Biological and Marine Sciences, University of Plymouth, Plymouth PL4 8AA (J.A.R.)

$\S$ Denotes equal contribution of authors

*e-mail: malcolm.bennett@nottingham.ac.uk (M.J.B.); hideyuki@ige.tohoku.ac.jp (H.T.) 
41 Plants can acclimate by using tropisms to link the direction of growth to

42 environmental conditions. Hydrotropism allows roots to forage for water, a process

43 known to depend on abscisic acid (ABA) but whose molecular and cellular basis

44 remains unclear. Here, we show that hydrotropism still occurs in roots after laser

45 ablation removed the meristem and root cap. Additionally, targeted expression studies reveal that hydrotropism depends on the ABA signalling kinase, SnRK2.2, and

47 the hydrotropism-specific MIZ1, both acting specifically in elongation zone cortical 48 cells. Conversely, hydrotropism, but not gravitropism, is inhibited by preventing 49 differential cell-length increases in the cortex, but not in other cell types. We conclude 50 that root tropic responses to gravity and water are driven by distinct tissue-based 51 mechanisms. In addition, unlike its role in root gravitropism, the elongation zone 52 performs a dual function during a hydrotropic response, both sensing a water potential gradient and subsequently undergoing differential growth. 
Tropic responses are differential growth mechanisms that roots use to explore the surrounding soil efficiently. In general, a tropic response can be divided into several steps, comprising perception, signal transduction, and differential growth. All of these steps have been well characterized for gravitropism, where gravity sensing cells in the columella of the root cap generate a lateral auxin gradient, whilst adjacent lateral root cap cells transport auxin to epidermal cells in the elongation zone, thereby triggering the differential growth that drives bending ${ }^{1-4}$. In gravi-stimulated roots, the lateral auxin gradient is transported principally by AUX1 and PIN carriers ${ }^{3-5}$.

Compared with gravitropism, the tropic response to asymmetric water availability, i.e., hydrotropism, has been far less studied. Previously, it was reported that surgical removal or ablation of the root cap reduces hydrotropic bending in pea ${ }^{6-8}$ and Arabidopsis thaliana ${ }^{9}$, suggesting that the machinery for sensing moisture gradients resides in the root cap. It has also been reported that hydrotropic bending occurs due to differential growth in the elongation zone ${ }^{7,10}$. However unlike gravitropism, hydrotropism in $A$. thaliana is independent of AUX1 and PIN-mediated auxin transport ${ }^{11,12}$. Indeed, roots bend hydrotropically in the absence of any redistribution of auxin detectable by auxin-responsive reporters ${ }^{13,14}$. Instead, root hydrotropism requires signalling by the hormone abscisic acid $(A B A)^{12}$. These findings imply that, compared to gravitropism, hydrotropism requires a distinct signalling mechanism ${ }^{15}$.

The involvement of $A B A$ in hydrotropism was initially suggested by aberrant responses in A. thaliana mutants deficient for ABA synthesis or response ${ }^{12}$. More recently, loss-of-function $A B A$ receptor and response mutants that are insensitive or hypersensitive to $A B A$ have been shown to be insensitive or hypersensitive to a hydrotropic stimulus, respectively ${ }^{16}$. In addition, hydrotropism in $A$. thaliana roots requires a gene called MIZU-KUSSEI1 $(M I Z 1)^{17}$, which is upregulated by application of $10 \mu \mathrm{M} \mathrm{ABA}^{18}$. Despite miz1 roots being oblivious to water potential gradients, they nevertheless bend like wild type in response to gravity ${ }^{17}$. The MIZ1 sequence contains a DUF617 domain that is conserved among the genomes of 
terrestrial plants, but absent in algae and animals, suggesting a role for hydrotropism in the evolution of land plants ${ }^{17}$. A functional MIZ1:MIZ1-GFP fusion protein is expressed in lateral root cap cells as well as cortex and epidermis cells in the meristem and elongation zone ${ }^{18,19}$. However it is unclear whether this broad expression pattern is necessary for MIZ1's function in hydrotropism or whether ABA signal transduction components in general have to be expressed in specific root tip tissues for a hydrotropic response. The present study describes a series of experiments in $A$. thaliana designed to identify the root tissues essential for a hydrotropic response. We report that MIZ1 and a key ABA signal-transduction component SnRK2.2 expressed specifically in the root cortex are sufficient to drive hydrotropism, and conversely that hydrotropism is blocked by inhibiting the ability of specifically the cortex to execute a differential growth response. Our results support a re-evaluation of hydrotropic signalling, revealing the importance of the cortex and the elongation zone for signal perception as well as bending.

\section{Results}

\section{The root meristem and columella are dispensable for hydrotropism}

To uncover which root cell types and zones are required during a hydrotropic response in $A$. thaliana roots, we ablated cells using a femtosecond laser. Successful ablation of the columella cells was confirmed by propidium iodide staining of root tissues (Fig. 1a, b) and hydro- and gravitropism assays performed as described previously ${ }^{11,17}$ (for details on hydrotropism assays used in this paper, see Supplementary Fig. 1). Whilst columella ablation successfully inhibited the gravitropic response as previously reported ${ }^{1}$, it did not inhibit the hydrotropic response (Fig. 1c, e). We suggest the discrepancy with earlier experiments arose from their being performed under adverse growth conditions, as indicated by roots elongating more than an order of magnitude slower than those used here. Importantly, the ablated roots in this study elongated at an equivalent rate as the intact roots 
throughout both gravitropism and hydrotropism assays (Fig. 1d, f). Further probing of the region necessary for stimulus perception showed that even when ablation encompassed essentially the entire meristem, hydrotropism was scarcely affected (Supplementary Fig. 2). Crucially, when seedlings with ablated root cap or meristem were placed in an assay system that lacked the moisture gradient, ablated roots responded in the same way as intact roots with only minimal bending, demonstrating that laser ablation per se did not induce a response that mimicked hydrotropism (Supplementary Fig. 2).

Because this apparent dispensability of the columella conflicts with previous results with laser ablation ${ }^{9}$, we independently validated the experiment in the split-agar system by excising the distal region of the root tip $(\sim 250 \mu \mathrm{m})$ manually. As with laser ablation, manual excision of columella and meristem did not induce bending in the absence of a water potential gradient (Supplementary Fig. 2), demonstrating root tip removal does not mimic a hydrotropism response. Most significantly, manual excision of columella and meristem did not disrupt hydrotropic bending in the presence of a water potential gradient, giving results comparable to whole roots (Supplementary Fig. 2). Whilst we cannot exclude the possibility that hydrotropic stimuli are perceived in the root cap when that tissue is present, our ablation and excision results demonstrate that roots are able to sense as well as respond to water potential gradients within the elongation zone.

\section{Root hydrotropism depends on the ABA signalling component SnRK2.2}

ABA represents a critical signal for numerous plant abiotic stress responses ${ }^{20}$ including root hydrotropism. ABA responses are mediated by a negative regulatory signalling module involving soluble receptors of the START-domain superfamily (PYR/PYL/RCARs), clade A, type 2C protein phosphatases (PP2Cs) and subclass III Snf1-related kinases $\left(\right.$ SnRK2s) ${ }^{20}$. ABA binds to PYR1/PYL/RCAR, which induces a conformational change that allows the receptor proteins to bind to, and thereby inhibit, PP2Cs ${ }^{21,22}$. PP2Cs dephosphorylate SnRK2s, suppressing their activity; thus SnRK2 activity increases in the presence of ABA due to PP2Cs being bound to the PYR1/PYL/RCAR ABA receptors ${ }^{23}$. 
When active, the SnRK2s phosphorylate transcription factors and other downstream $\operatorname{target}^{20,23}$.

To investigate how ABA controls hydrotropism, we characterised a double mutant lacking the ABA signalling kinases SnRK2.2 and SnRK2.324. Although retaining some ABA responsiveness, this double mutant was selected for experiments because, in contrast to most mutants in ABA perception, it is neither dwarfed nor wilty. We initially assayed hydrotropism in a split-agar-based system ${ }^{25}$. Hydrotropism in the snrk2.2 snrk2.3 double mutant was strongly attenuated, but was restored in the snrk2.2 snrk2.3 double mutant expressing the SnRK2.2 gene under the control of its own promoter (Fig. 2b). Identical results were obtained using a moisture gradient in air hydrotropic assay (Supplementary Fig. 5). Hence, the SnRK2.2 kinase appears to be required for hydrotropism.

As the snrk2.2 snrk2.3 double mutant had slightly shorter roots and a reduced growth rate compared to wild type (Supplementary Fig. 3), we compared the growth rates of the double mutant on half strength Murashige and Skoog (MS) medium and hydrotropism plates and found them to be comparable (Supplementary Fig. 3), ruling out hypersensitivity of the snrk2.2 snrk2.3 double mutant to sorbitol. In addition, we performed split-agar hydrotropism assays with younger wild-type seedlings to assess whether a reduction in tip angle was caused simply by a reduced root growth rate. Roots bent with similar kinetics despite differences in length and growth rate, indicating that hydrotropic bending is not proportional to root growth rate (Supplementary Fig. 3).

\section{Hydrotropism requires SnRK2.2 signalling only in the root cortex}

To gain insight into the tissue specificity of hydrotropism, we created a translational GFP fusion to the SnRK2.2 genomic sequence and expressed the reporter in the snrk2.2 snrk2.3 double mutant background. In the resulting lines, roots regained wild type sensitivity to $10 \mu \mathrm{M}$ ABA (Supplementary Fig. 4) and bent hydrotropically in the moisture gradient in air assay (but not the split-agar assay) (Supplementary Fig. 4). We assume that the differences in hydrotropic response obtained using the different assays could be due to the moisture in 
air gradient providing a steeper water potential gradient than the split-agar assays. Hence, the translational reporter appeared partially functional. Using confocal imaging SnRK2.2GFP signal was detected in nuclear and cytoplasmic compartments, consistent with the subcellular localisation of its known regulatory targets ${ }^{26,27}$. Moreover, at the tissue scale, SnRK2.2:SnRK2.2-GFP was ubiquitously expressed throughout the root apex, including root cap and elongation zone (Fig. 2c).

To pinpoint the root tissue where SnRK2.2 is required during a hydrotropic response, we expressed the SnRK2.2 genomic sequence in the snrk2.2 snrk2.3 double mutant background using a suite of tissue- and zone-specific promoters. SnRK2.2 expressed under

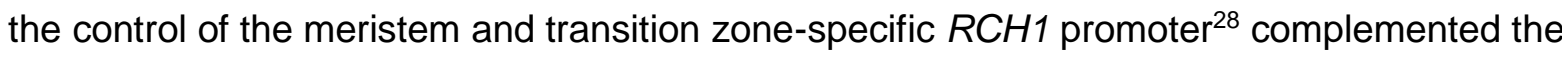
snrk2.2 snrk2.3 hydrotropic defect (Fig. 2d). Surprisingly, rescue failed when SnRK2.2 was expressed specifically in the root cap (SOMBRERO $\left.{ }^{29}, S M B: S n R K 2.2\right)$, epidermis and lateral root cap (WEREWOLF ${ }^{30}$, WER:SnRK2.2), or endodermis (SCARECROW ${ }^{31}$, SCR:SnRK2.2) (Fig. 2d). In contrast, double mutant roots bent hydrotropically as the wild type when expressing SnRK2.2 in just the cortex (Co2 $\left.{ }^{32}, C_{02}: S n R K 2.2\right)$ (Fig. 2d). SnRK2.2 expression levels in the Co2:SnRK2.2 line were low in comparison to non-rescuing epidermal, lateral root cap or endodermal driven lines, demonstrating that mutant rescue is not simply a dose effect (Supplementary Fig. 5). In addition, we confirmed the hydrotropism response of the Co2:SnRK2.2 line using the moisture in air gradient assay (Supplementary Fig. 5). Hence, root hydrotropism appears to require the ABA response machinery specifically in the cortex.

\section{Cortex-specific MIZ1 expression rescues the miz1 hydrotropic defect}

To independently assess tissue specificity for the hydrotropic response, we determined which tissues require MIZ1, a protein previously identified as essential for hydrotropism and localized to cortex, epidermis, and lateral root cap ${ }^{19}$. We used various promoters to express MIZ1-GFP in specific tissues in the miz1 background (Supplementary Fig.6). When constructs that included the MIZ1 terminator were used, MIZ1-GFP expression driven by $\mathrm{RCH} 1$ was detected in the meristem, by $S M B$ in the root cap, by $S C R$ in the 
endodermis, and by COR and by Co2 in the cortex, all as expected ${ }^{28,29,31-33}$ (Supplementary Fig. 6). Compared to SCR or Co2, the COR promoter drove MIZ1-GFP expression farther into the elongation zone. In contrast, the WER promoter drove MIZ1-GFP expression not only in the epidermis and lateral root cap, as expected ${ }^{30}$, but also in the cortex. Like COR, expression from WER continued well into the elongation zone. Note that none of these constructs altered root growth rate appreciably (Supplementary Fig. 6).

Using the tissue-specific MIZ1-GFP constructs, we assayed hydrotropism using the moisture gradient in air method, which gave approximately $80^{\circ}$ bending after 12 hours. As expected, hydrotropic bending was fully rescued by expressing MIZ1-GFP under the MIZ1 promoter (Supplementary Fig. 6). In contrast, little or no hydrotropic curvature resulted when MIZ1-GFP was expressed in root cap (SMB), in endodermis (SCR), or in the meristem $(R C H 1)$. Mutant complementation was only partial using Co2 to drive MIZ1-GFP expression, but rescue was complete employing either WER or COR promoters, revealing a requirement for MIZ1 in the elongation zone (Supplementary Fig. 6). Mutant rescue was also complete when MIZ1-GFP expression was driven by the PIN2 promoter, which like WER, drives expression in lateral root cap, epidermis and cortex, which for the latter tissues continues well into the elongation zone (Fig. 2 e-g). Finally, when WER-driven expression was removed from the cortex, which happened if the native MIZ1 terminator was replaced by a terminator from a heat-shock protein (HSP), miz1 rescue essentially failed (Fig. 2e,g). Identical responses for WER- and PIN2-driven MIZ1-GFP expression were obtained using the split-agar assay (Supplementary Fig. 5). Taken together, these results show that hydrotropic bending requires MIZ1 expression specifically in the root cortex and that the expression domain must span at least part of the elongation zone. This conclusion is consistent with laser ablation and SnRK2.2 expression experiments that, when taken collectively, establishes the functional importance of the cortex within the elongation zone for the hydrotropic response. 


\section{Low levels of ABA promote root elongation}

Root cortical cells abut the endodermis (Fig. 2a), a recently reported site of $A B A$ accumulation in roots ${ }^{34}$. Hence, ABA response machinery in the cortex would be ideally positioned to sense lateral movement of ABA from the endodermis into outer root tissues, and presumably triggering growth responses. In roots, whilst high ABA levels inhibit growth $^{24}$, low levels of this hormone promote elongation at low water potential ${ }^{35-37}$. To understand the ABA-dependent growth mechanism underlying hydrotropism, we next investigated the effect of low doses of ABA on root growth. Transferring seedlings onto 100 nM ABA stimulated root growth rate in the wild type but had minimal effect on snrk2.2 snrk2.3 (Fig. 3a, Supplementary Fig. 7). Comparing meristem and elongation zone of those roots, $100 \mathrm{nM}$ ABA appeared to change neither the length nor cell number within the meristem but significantly increased elongation-zone length in wild type and Co2:SnRK2.2 complementation lines (Fig. 3c, Supplementary Fig. 7). The increased root growth rate was accompanied by both an increased rate of cell production and an increased mature cell length (Supplementary Fig. 7, Fig. 3b). Taken together, these data suggest that low doses of ABA in these non-stressed plants stimulate rates of cell division and elemental elongation. To examine tissue specificity in the promotion of root growth by ABA, we analysed nuclear ploidy of specific tissues by performing cell sorting and DNA-content measurements. Significantly, 100 nM ABA stimulated endoreplication specifically in root cortical cells, as evidenced by the increased fraction of $8 \mathrm{C}$ nuclei at the expense of $4 \mathrm{C}$ (Fig. 3e). In contrast, $100 \mathrm{nM}$ ABA had little if any effect on endoreplication in either atrichoblast or endodermal cells (Fig. 3d, f). Hence, ABA appears to specifically trigger changes in cell cycle machinery in just the cortex, consistent with a fundamental role for this tissue in mediating hydrotropism.

\section{Hydrotropism is driven by differential cortical cell expansion}

One might question whether an asymmetry of growth-promoting mechanisms within a single tissue could provide sufficient mechanical leverage to trigger root curvature. To 
explore whether such changes in the dynamics of cortical cells are sufficient to drive root

247 bending during hydrotropism, we developed a mathematical model (see Methods and

248 Supplementary Note 1), taking advantage of recent theoretical work that successfully recapitulates the root's growth rate profile by ascribing distinct mechanical contributions to the various tissues ${ }^{38}$. For a short period following exposure to the water potential gradient, a small group of cortical cells on the dry side of the root were treated as undergoing early entry into rapid elongation, changing their mechanical properties to be the same as cells in the elongation zone. This differential elongation, coupled with the cell-to-cell adhesion typical for plant cells, caused the root midline to bend in this region (Supplementary Fig. 8).

To assess the model further, we quantified the growth kinetics of hydrotropically bending roots by using image analysis, resolving both elemental elongation and curvature. These experimental data resembled the evolution of root tip angle predicted by the model cortex.

If hydrotropic bending is driven by an asymmetric expansion of cortical cells in the elongation zone, we reasoned that hydrotropism could be blocked by interfering with the orderly progression of cells through the growth zone. To test this, we took advantage of the SIM were enlarged (Fig. 4a-c) but cells in adjacent tissues were not detectably affected and were of similar length to cells of roots expressing only the GFP marker (Fig. 4d-e). Next, we tested each tissue-specific, SIM over-expressing line for hydrotropism. Roots overexpressing SIM in root epidermis or endodermis bent indistinguishably from the parental lines, whereas SIM overexpression in the cortex blocked root hydrotropic bending (Fig. 4f). 
274 In contrast, roots of every SIM overexpression line retained a wild-type response to gravity

275 (Fig. 4g), revealing that SIM overexpression in the cortex did not simply prevent all 276 differential root growth processes.

\section{Discussion}

We report that root tropic responses to gravity and water are driven by distinct molecular and tissue-based mechanisms. In the case of gravity, root re-orientation is sensed by columella cells at the root tip ${ }^{1}$, triggering the formation of a lateral auxin gradient across the root with higher concentrations on the lower side of the $\operatorname{root}^{40,41}$. This auxin gradient is then transported via the lateral root cap to epidermal cells in the elongation zone ${ }^{3}$ where it elicits downward root bending by stimulating expansion on the upper side and inhibiting it on the lower-side ${ }^{42}$. In contrast, here, laser ablation experiments demonstrate that perceiving a water potential gradient and fully responding thereto requires neither meristem, lateral root cap, nor columella (Fig. 1). Hence, unlike its role in root gravitropism, the elongation zone is able to perform a dual function during a hydrotropic response, both sensing a water potential gradient and undergoing differential growth. This conclusion stands despite the possibility of meristem and root cap participating in hydrotropism in intact roots, for example by integrating signals from water and gravity.

We also confirm that root hydrotropism uses the hormone $A B A$ and that the $A B A$ signal transduction components SnRK2.2 and SnRK2.3 play a key role regulating root reorientation. Surprisingly, targeted SnRK2.2 expression studies in snrk2.2 snrk2.3 (Fig. 2) revealed the critical importance during hydrotropism of ABA response machinery just in the cortex. The importance of this specific root tissue for hydrotropism was further supported by the response depending on cortical expression of MIZ1 (Fig. 2). Taken together, our results demonstrate that $\mathrm{ABA}$ and MIZ1 responses in the cortex of the root elongation zone play a central role in hydrotropic response of $A$. thaliana roots (Fig. 5). Hence, root gravitropic and hydrotropic responses are driven by distinct signals and tissue-based mechanisms. 
301 Consistent with our conclusion, Krieger et al. ${ }^{43}$ recently described the opposing effect of reactive oxygen species on these tropic responses and the distinct positions at which roots bend during gravitropic and hydrotropic responses.

A key question for hydrotropic research is to understand how a modest gradient in water potential across the root is perceived (and presumably amplified) into a growth response. Mechano-sensing, differential movement of water, ions or signalling molecules all represent likely candidates, but detection methods that are more sensitive than those currently available will be necessary to get to the root of this plant environmental response.

\section{METHODS}

\section{Ablation of root-tip cells using laser-microscopy systems}

For micro-beam laser irradiation, 4-day-old seedlings were aligned in a micro-chamber comprising two glass coverslips $\left(25 \times 60 \mathrm{~mm}^{2}\right.$ and $24 \times 24 \mathrm{~mm}^{2}$, Matsunami) and a seal (TaKaRa Slide Seal for in situ PCR, Takara Bio). The micro-chamber was filled with lowmelting agar (0.5x MS medium, $0.4 \%(\mathrm{w} / \mathrm{v})$ sucrose [Wako Pure Chemical Industries], $0.2 \%$ (w/v) low-melting agarose [SeaPlaque; FMC BioProducts]). These samples were put on the stage of a microscope (Nikon ECLIPSE TiE, Nikon) and irradiated with a $\mathrm{N}_{2}$ pulsed microbeam laser through Coumarin 440 with an averaged power of $330 \mathrm{~kW}$ for a 3 to 5 nanosecond pulse (MicroPoint PIJ-3-1; Andor Technology). For femtosecond laser irradiation, seedlings were placed on $0.5 x$ MS medium on a glass slide. Amplified femtosecond laser pulses from a re-generatively amplified Ti:sapphire femtosecond laser system (IFRIT; $780 \pm 5 \mathrm{~nm}, 230 \mathrm{fs},<1 \mathrm{~mJ} /$ pulse, $1 \mathrm{kHz}$, Cyber Laser Inc.) were focused onto root cap cells through a 10x objective lens (UPlanSApo NA 0.4, Olympus) on a confocal laser scanning microscope (FV1000-BX51, Olympus). Laser pulses (200) were detected with a mechanical shutter (gate time: $200 \mathrm{~ms}$ ) and delivered to the sample. The laser pulse was collimated by dual convex lenses before the microscope, and the laser focal point was tuned to the plane of the image. The diameter of the laser focal point, which is consistent with the beam waist, was about $1 \mu \mathrm{m}$. A neutral density filter was put between the laser and 
microscope and used to tune the laser pulse energy to around $400 \mathrm{~nJ} /$ pulse, which is about 4 times larger than the threshold energy for cavitation bubble generation in water (100 $\mathrm{nJ} /$ pulse). Laser-ablated seedlings were incubated on $0.5 \mathrm{x}$ MS medium for $1 \mathrm{~h}$ in a vertical position before performing further assays.

\section{Root tropism and growth assays}

The hydrotropism assay shown in Fig. 1 and Supplementary Fig. 5b-e were performed as described previously using a split-agar system with $812 \mathrm{mM}$ sorbitol ${ }^{11}$. Gravitropism assays shown in Fig. 1 and Supplementary Fig. 2 were performed using 1\% agar medium with or without $0.5 x \mathrm{MS}$ medium as described previously ${ }^{17}$. Hydrotropism assays shown in Fig. $2 \mathrm{~g}$ and Supplementary Fig. 2g-I, 4e-f, $5 \mathrm{f}-\mathrm{g}$ and 6 were performed using a moisture gradient in air as described previously ${ }^{11}$. Four-day-old seedlings were used for all tropism assays described above.

Hydrotropism assays shown in Fig. 2b,d and 4 and Supplementary Fig. 2m, 3, 4d and 8 were performed as previously described ${ }^{25}$ using 5-day-old seedlings in a split-agar system with $400 \mathrm{mM}$ sorbitol.

For gravitropism assays shown in Fig. 4, 5-day-old seedlings were transferred to new plates containing $0.5 \mathrm{x}$ MS medium with $1 \%$ agar. After acclimatisation for 2 hours in the controlled environment room, plates were rotated by $90^{\circ}$. Images of seedlings were acquired using an automated imaging platform ${ }^{44}$ and root tip angle and length determined using the Fiji image processing package (http://fiji.sc/Fiji).

For assessing root growth response to ABA, 5-day-old seedlings were transferred to new plates containing $0.5 \mathrm{x}$ MS medium with the indicated amount of ABA (Sigma). To determine meristem cell number and length, longitudinal images of root tips clearly showing the cortex cell file were taken with a confocal laser scanning microscope, using propidium iodide to stain cell walls. Starting from the quiescent centre (QC), the length of individual cortex cells was determined using the Cell-o-Tape macro ${ }^{45}$ for Fiji. The mean length of meristem cells was calculated using ten cells from the rapid amplifying region of the meristem (cells 10-19 
counting shootward from the QC), and the end of the meristem deemed to have been reached when consecutive cells had reached or exceeded the mean length by two. Cell production rates were calculated as previously described ${ }^{46}$.

\section{Modelling root bending}

A mechanical model has been developed to describe hydrotropism-associated root bending. The approach ${ }^{38}$ exploits the large aspect ratio of the root, which allows a relatively simple description of bending in terms of the stretch and curvature of the root midline. A viscoplastic constitutive relation is adopted (viscous flow where the yield stress is exceeded), with the yield stress of cortical cells on the dry side of the root modified in response to a hydrotropic stimulus; the resulting partial differential equations for the dependence of midline stretch and curvature in terms of time and arc length are solved numerically by a finite-difference approach. Further details are given in the Supplementary Note 1, Section 2.

\section{Data availability}

The data that support the findings of this study are available from the corresponding authors upon request.

\section{References}

1 Blancaflor, E. B., Fasano, J. M. \& Gilroy, S. Mapping the functional roles of cap cells in the response of Arabidopsis primary roots to gravity. Plant Physiol 116, 213-222 (1998).

2 Ottenschlager, I. et al. Gravity-regulated differential auxin transport from columella to lateral root cap cells. P Natl Acad Sci USA 100, 2987-2991, doi:10.1073/pnas.0437936100 (2003).

3 Swarup, R. et al. Root gravitropism requires lateral root cap and epidermal cells for transport and response to a mobile auxin signal. Nat Cell Biol 7, 1057-1065, doi:10.1038/ncb1316 (2005). 
4 Rahman, A. et al. Gravitropism of Arabidopsis thaliana roots requires the polarization of PIN2 toward the root tip in meristematic cortical cells. Plant Cell 22, 1762-1776, doi:10.1105/tpc.110.075317 (2010).

$5 \quad$ Friml, J. Subcellular trafficking of PIN auxin efflux carriers in auxin transport. Eur J Cell Biol 89, 231-235, doi:10.1016/j.ejcb.2009.11.003 (2010).

6 Jaffe, M. J., Takahashi, H. \& Biro, R. L. A pea mutant for the study of hydrotropism in roots. Science 230, 445-447, doi:10.1126/science.230.4724.445 (1985).

7 Takahashi, H. \& Suge, H. Root hydrotropism of an agravitropic pea mutant, ageotropum. Physiol Plantarum 82, 24-31 (1991).

8 Takahashi, H. \& Scott, T. K. Intensity of hydrostimulation for the induction of root hydrotropism and its sensing by the root cap. Plant Cell Environ 16, 99-103, doi:DOI 10.1111/j.1365-3040.1993.tb00850.x (1993).

9 Miyazawa, Y. et al. Effects of locally targeted heavy-ion and laser microbeam on root hydrotropism in Arabidopsis thaliana. J Radiat Res 49, 373-379, doi:10.1269/jrr.07131 (2008).

10 Miyamoto, N., Ookawa, T., Takahashi, H. \& Hirasawa, T. Water uptake and hydraulic properties of elongating cells in hydrotropically bending roots of Pisum sativum $\mathrm{L}$. Plant Cell Physiol 43, 393-401, doi:DOI 10.1093/pcp/pcf046 (2002).

11 Kaneyasu, T. et al. Auxin response, but not its polar transport, plays a role in hydrotropism of Arabidopsis roots. J Exp Bot 58, 1143-1150, doi:10.1093/jxb/erl274 (2007).

12 Takahashi, N., Goto, N., Okada, K. \& Takahashi, H. Hydrotropism in abscisic acid, wavy, and gravitropic mutants of Arabidopsis thaliana. Planta 216, 203-211, doi:10.1007/s00425-002-0840-3 (2002).

13 Takahashi, H., Miyazawa, Y. \& Fujii, N. Hormonal interactions during root tropic growth: hydrotropism versus gravitropism. Plant Mol Biol 69, 489-502, doi:10.1007/s11103-008-9438-x (2009).

14 Shkolnik, D., Krieger, G., Nuriel, R. \& Fromm, H. Hydrotropism: Root Bending Does Not Require Auxin Redistribution. Mol Plant 9, 757-759, doi:10.1016/j.molp.2016.02.001 (2016).

15 Shkolnik, D. \& Fromm, H. The Cholodny-Went theory does not explain hydrotropism. Plant Sci 252, 400-403, doi:10.1016/j.plantsci.2016.09.004 (2016).

16 Antoni, R. et al. PYRABACTIN RESISTANCE1-LIKE8 Plays an Important Role for the Regulation of Abscisic Acid Signaling in Root. Plant Physiology 161, 931-941, doi:10.1104/pp.112.208678 (2013).

17 Kobayashi, A. et al. A gene essential for hydrotropism in roots. P Natl Acad Sci USA 104, 4724-4729, doi:10.1073/pnas.0609929104 (2007).

18 Moriwaki, T., Miyazawa, Y., Fujii, N. \& Takahashi, H. Light and abscisic acid signalling are integrated by MIZ1 gene expression and regulate hydrotropic response in roots of Arabidopsis thaliana. Plant Cell Environ 35, 1359-1368, doi:10.1111/j.1365-3040.2012.02493.x (2012).

19 Moriwaki, T., Miyazawa, Y., Kobayashi, A. \& Takahashi, H. Molecular Mechanisms of Hydrotropism in Seedling Roots of Arabidopsis thaliana (Brassicaceae). Am J Bot 100, 25-34, doi:10.3732/ajb.1200419 (2013).

20 Cutler, S. R., Rodriguez, P. L., Finkelstein, R. R. \& Abrams, S. R. Abscisic Acid: Emergence of a Core Signaling Network. Annu Rev Plant Biol 61, 651-679, doi:10.1146/annurev-arplant-042809-112122 (2010).

$21 \mathrm{Ma}, \mathrm{Y}$. et al. Regulators of PP2C Phosphatase Activity Function as Abscisic Acid Sensors. Science 324, 1064-1068, doi:10.1126/science.1172408 (2009).

22 Park, S. Y. et al. Abscisic Acid Inhibits Type 2C Protein Phosphatases via the PYR/PYL Family of START Proteins. Science 324, 1068-1071, doi:10.1126/science.1173041 (2009).

23 Fujii, $\mathrm{H}$. et al. In vitro reconstitution of an abscisic acid signalling pathway. Nature 462, 660-664, doi:10.1038/nature08599 (2009). 
24 Fujii, H., Verslues, P. E. \& Zhu, J. K. Identification of two protein kinases required for abscisic acid regulation of seed germination, root growth, and gene expression in Arabidopsis. Plant Cell 19, 485-494, doi:10.1105/tpc.106.048538 (2007).

25 Antoni, R., Dietrich, D., Bennett, M. J. \& Rodriguez, P. L. Hydrotropism: Analysis of the Root Response to a Moisture Gradient. Methods Mol Biol 1398, 3-9, doi:10.1007/978-1-4939-3356-3_1 (2016).

26 Kline, K. G., Barrett-Wilt, G. A. \& Sussman, M. R. In planta changes in protein phosphorylation induced by the plant hormone abscisic acid. P Natl Acad Sci USA 107, 15986-15991, doi:10.1073/pnas.1007879107 (2010).

27 Wang, P. et al. Quantitative phosphoproteomics identifies SnRK2 protein kinase substrates and reveals the effectors of abscisic acid action. Proc Natl Acad Sci U S A 110, 11205-11210, doi:10.1073/pnas.1308974110 (2013).

28 Casamitjana-Martinez, E. et al. Root-specific CLE19 overexpression and the sol1/2 suppressors implicate a CLV-like pathway in the control of Arabidopsis root meristem maintenance. Curr Biol 13, 1435-1441, doi:10.1016/S0960-9822(03)00533-5 (2003).

29 Willemsen, V. et al. The NAC Domain Transcription Factors FEZ and SOMBRERO Control the Orientation of Cell Division Plane in Arabidopsis Root Stem Cells. Dev Cell 15, 913-922, doi:10.1016/j.devcel.2008.09.019 (2008).

30 Lee, M. M. \& Schiefelbein, J. WEREWOLF, a MYB-related protein in Arabidopsis, is a position-dependent regulator of epidermal cell patterning. Cell 99, 473-483, doi:Doi 10.1016/S0092-8674(00)81536-6 (1999).

31 Wysocka-Diller, J. W., Helariutta, Y., Fukaki, H., Malamy, J. E. \& Benfey, P. N. Molecular analysis of SCARECROW function reveals a radial patterning mechanism common to root and shoot. Development 127, 595-603 (2000).

32 Heidstra, R., Welch, D. \& Scheres, B. Mosaic analyses using marked activation and deletion clones dissect Arabidopsis SCARECROW action in asymmetric cell division. Genes \& Dev 18, 1964-1969, doi:10.1101/gad.305504 (2004).

33 Lee, J. Y. et al. Transcriptional and posttranscriptional regulation of transcription factor expression in Arabidopsis roots. P Natl Acad Sci USA 103, 6055-6060, doi:10.1073/pnas.0510607103 (2006).

34 Ondzighi-Assoume, C. A., Chakraborty, S. \& Harris, J. M. Environmental Nitrate Stimulates Abscisic Acid Accumulation in Arabidopsis Root Tips by Releasing It from Inactive Stores. Plant Cell 28, 729-745, doi:10.1105/tpc.15.00946 (2016).

35 Sharp, R. E., Wu, Y. J., Voetberg, G. S., Saab, I. N. \& Lenoble, M. E. Confirmation That Abscisic-Acid Accumulation Is Required for Maize Primary Root Elongation at Low Water Potentials. J Exp Bot 45, 1743-1751 (1994).

$36 \mathrm{Xu}, \mathrm{W}$. F. et al. Abscisic acid accumulation modulates auxin transport in the root tip to enhance proton secretion for maintaining root growth under moderate water stress. New Phytol 197, 139-150, doi:10.1111/nph.12004 (2013).

37 Rowe, J. H., Topping, J. F., Liu, J. \& Lindsey, K. Abscisic acid regulates root growth under osmotic stress conditions via an interacting hormonal network with cytokinin, ethylene and auxin. New Phytol 211, 225-239, doi:10.1111/nph.13882 (2016).

38 Dyson, R. J. et al. Mechanical modelling quantifies the functional importance of outer tissue layers during root elongation and bending. New Phytol 202, 1212-1222, doi:10.1111/nph.12764 (2014).

39 Churchman, M. L. et al. SIAMESE, a plant-specific cell cycle regulator, controls endoreplication onset in Arabidopsis thaliana. Plant Cell 18, 3145-3157, doi:10.1105/tpc.106.044834 (2006).

40 Brunoud, G. et al. A novel sensor to map auxin response and distribution at high spatio-temporal resolution. Nature 482, 103-U132, doi:10.1038/nature10791 (2012).

41 Band, L. R. et al. Root gravitropism is regulated by a transient lateral auxin gradient controlled by a tipping-point mechanism. Proc Natl Acad Sci U S A 109, 4668-4673, doi:10.1073/pnas.1201498109 (2012). 
Mullen, J. L., Ishikawa, H. \& Evans, M. L. Analysis of changes in relative elemental growth rate patterns in the elongation zone of Arabidopsis roots upon gravistimulation. Planta 206, 598-603, doi:DOI 10.1007/s004250050437 (1998).

43 Krieger, G., Shkolnik, D., Miller, G. \& Fromm, H. Reactive Oxygen Species Tune Root Tropic Responses. Plant Physiology 172, 1209-1220, doi:10.1104/pp.16.00660 (2016).

44 Wells, D. M. et al. Recovering the dynamics of root growth and development using novel image acquisition and analysis methods. Philos $T R$ Soc $B$ 367, 1517-1524, doi:10.1098/rstb.2011.0291 (2012).

45 French, A. P. et al. Identifying biological landmarks using a novel cell measuring image analysis tool: Cell-o-Tape. Plant Methods 8, 7 doi:Artn 710.1186/1746-4811-87 (2012).

46 Baskin, T. I. Patterns of root growth acclimation: constant processes, changing boundaries. WIREs Dev Biol 2, 65-73, doi:10.1002/wdev.94 (2013).

\section{ACKNOWLEDGEMENTS}

The authors thank Caroline Howells, Kamal Swarup and Morag Whitworth for technical assistance, Jian-Kang Zhu for providing snrk2.2 snrk2.3 seeds, Wim Grunewald for pDONRL1-GAL4-VP16-R2, Sumika Tsukinoki for generating WER:MIZ1-GFP(HSPter) and PIN2:MIZ1-GFP(HSPter) transgenic plants and acknowledge the following funding agencies for financial support: D.D., J.F., R.A., T. N., D.W., S.T., C.S., S.M., M.R.O., L.R.B., R.D., O.J., J.K., J.R., T.B. and M.J.B. thank the Biological and Biotechnology Science Research Council (BBSRC) for responsive mode and CISB awards to the Centre for Plant Integrative Biology; D.W., C.S., S.M., M.R.O., J.K., T.P. and M.J.B. thank the European Research Council (ERC) for FUTUREROOTS project funding; L.R.B. thanks the Leverhulme Trust for an Early Career Fellowship; V.B., R.B. and L.D.V are supported by grants of the Research Foundation Flanders (G.002911N). R.B. and M.J.B. thank the Royal Society for Newton and Wolfson Research Fellowship awards; R.A., T.I.B. and M.J.B. thank the FP7 Marie-Curie Fellowship Scheme; R.D. thanks the Engineering and Physical Sciences Research Council, J.D. and M.J.B. thank the Gll scheme; and V.B., R.B., L.D.V. and M.J.B. thank the Interuniversity Attraction Poles Programme (IUAP P7/29 "MARS"), initiated by the Belgian Science Policy Office. R.B.P was funded by grants from the Knut and Alice Wallenberg Foundation. This work was also supported by a Grant-in-Aid for Scientific Research on Innovative Areas (No. 22120004) from the Ministry of Education, Culture, Sports, Science and Technology (MEXT) 
of Japan to H.T., a Grant-in-Aid for Young Scientists (B) (No. 26870057) from the Japan

529 Society for the Promotion of Science (JSPS) to A.K., a Grant-in-Aid for Scientific Research on 530 Innovative Areas (No. 22120002) from the Ministry of Education, Culture, Sports, Science and 531 Technology (MEXT), Japan to A.N., a Grant-in-Aid for Scientific Research on Innovative Areas 532 (No. 22120010) from the Ministry of Education, Culture, Sports, Science and Technology 533 (MEXT), Japan to Y.H. and the Funding Program for Next-Generation World-Leading 534 Researchers (GS002) to Y.M. L.P. was financially supported by a scholarship from the 535 Japanese government. T.-W.B. was financially supported by the Funding Program for Next536 Generation World-Leading Researchers (GS002) and the Grant-in-Aid for Scientific Research 537 on Innovative Areas (No. 22120004).

538

\section{AUTHOR CONTRIBUTIONS}

D.D., L.P., A.K., J.F., V.B., R.B., R.A., T.N., S.H., T.-W.B., Y.M., D.M.W., S.T., C.J.S. performed experimental work and data analysis and mathematical modelling. D.M.W., M.R.O., L.R.B., R.D., O.J., J.R.K., S.J.M., J.R., R.B., J.D., P.L.R., T.I.B., T.P., L.D.V., N.F., Y.M., A.N., Y.H., H.T. and M.J.B. oversaw project planning and discussed experimental results and modelling simulations. D.D., L.P., A.K., N.F., Y.M., T.I.B., H.T. and M.J.B. wrote the paper.

\section{ADDITIONAL INFORMATION}

Supplementary information is available for this paper. Reprints and permissions information are available at www.nature.com/reprints. Correspondence and requests for materials should be addressed to H.T. or M.J.B.

\section{COMPETING FINANCIAL INTERESTS}

The authors declare no competing financial interests. 
557 Confocal fluorescence micrograph of propidium iodide-stained primary root tips before (a) and after (b) femtosecond-laser ablation of the columella, scale bar $=100 \mu \mathrm{m}$. Time-course study of root gravitropic curvature (c) and root growth (d). In c, $0^{\circ}$ equals horizontal. Timecourse study of root hydrotropic curvature (e) and root growth (f). In e, $0^{\circ}$ equals vertical. The hydrotropism assay was performed using the split-agar system with $812 \mathrm{mM}$ sorbitol. Values are mean \pm SEM of a representative experiment, $n=3-6$, from three independent experiments. Asterisks indicate statistically significant differences $\left({ }^{*} p<0.05,{ }^{* \star} p<0.01\right.$, Student's $t$-test).

Figure 2 ABA signalling in the cortex is crucial for root hydrotropism

a Schematic drawing indicating tissues in the root tip, grey: lateral root cap, red: epidermis, green: cortex, yellow: endodermis. b Kinetics of hydrotropic curvature after transferring seedlings to split-agar plates with $400 \mathrm{mM}$ sorbitol. Values are mean $\pm \mathrm{SEM}, n=29-40$. c Expression of SnRK2.2:SnRK2.2-GFP in the root tip, scale bar $=100 \mu \mathrm{m}$. d Hydrotropic curvature $12 \mathrm{~h}$ after transfer to split-agar plates with $400 \mathrm{mM}$ sorbitol. Values are mean \pm SEM, $n=24-31$. Different letters indicate statistically significant differences $(p<0.05$, Fisher's Least Significant Difference (LSD)). e, $\mathbf{f}$ Expression pattern of MIZ1-GFP fusion protein under control of (e) the WER and (f) PIN2 promoters with HSP terminator. Left-hand image shows an over lay of fluorescence from GFP (green) and PI (red), right-hand image shows GFP only. Arrowhead indicates the approximate rootward boundary of the elongation zone, scale bar $=100 \mu \mathrm{m}$. g Hydrotropic curvature $12 \mathrm{~h}$ after transfer of seedlings to the moisture gradient in air assay system. Values are mean \pm SEM of three independent experiments, $n=35-44$. Different letters indicate statistically significant differences $(p<0.05$, Tukey Honest Significant Difference (HSD) test). Col, $A$. thaliana Columbia-0 accession. 

a - c Root growth and histology. a Root growth without (0.5x MS) or with 100 nM ABA $24 \mathrm{~h}$

584

after transfer. Values are mean of three experiments \pm SD, $n=12-40$. b, c Seedlings treated as in (a) were stained with propidium iodide and images taken with a confocal microscope. $\mathbf{b}$ Cell length of mature cortex cells. Values are mean \pm SD, $n=18-47$ cells for 10 roots per line and treatment. c Meristem length was determined using Cell-o-Tape and an arithmetic method to determine the meristem end. Elongation zone length was determined by measuring the distance from the end of the lateral root cap until the first root hair bulge. Values are mean $\pm \mathrm{SD}, n=11-28$. For a $-\mathrm{c}$ : * statistically significant different $(p<0.01$, Student's $t$-test). $\mathbf{d}$ - $\mathbf{f}$ Endoreplication. DNA content of nuclei isolated from (d) the epidermis (non-hair cells), (e) cortex and (f) endodermis of roots treated for $24 \mathrm{~h}$ without $(0.5 \mathrm{x}$ MS, light bars) or with $100 \mathrm{nM}$ ABA (dark bars). Values are mean $\pm \mathrm{SD}$. For $\mathrm{d}-\mathrm{f}$ : * statistically significant different $(p<0.05$, Student's $t$-test). Col, $A$. thaliana Columbia- 0 accession.

\section{Figure 4 Inhibition of differential cell elongation in the cortex prevents hydrotropism}

\section{but not gravitropism}

a - c Confocal images of root tips co-expressing SIM and NLS-GFP (green) in (a) epidermis, (b) cortex, (c) endodermis. Cell walls were stained with propidium iodide (white). In c, two images of the same root are shown, for better visualization of the endodermis cell file. Scale bars for a - c = $100 \mu \mathrm{m}$. d, e Quantification of cell lengths for epidermis, cortex and endodermis files in the meristem. Values are mean $\pm \mathrm{SD}, n=7-52$ cells from three plants for each line and tissue. $\mathrm{f}$ Hydrotropic curvature $10 \mathrm{~h}$ after transfer to split-agar plates with 400 $\mathrm{mM}$ sorbitol. Values are mean $\pm 2 x \operatorname{SEM}, n=14-15$ for parental lines (GL2, Co2, En7) and $n$ = 56 for SIM expression lines (GL2>>SIM, Co2>>SIM, En7>>SIM). Different letters indicate statistically significant differences ( $p<0.05$, Fisher's LSD). g Gravitropic curvature 8 h after plates were rotated by $90^{\circ}$. Values are mean $\pm 2 x$ SEM, $n=30-31$.

\section{Figure 5 Conceptual model for root hydrotropism}


611 SnRK2.2 and MIZ1 expression in cortex cells of the transition and elongation zone are

612 required to mediate the ABA-dependent differential growth response to a water potential

613 gradient. Perception of the water potential gradient does not require tissues in the root cap

614 or meristem, but takes place in the transition and elongation zones where the differential

615 growth response occurs.

616 
Figure 1
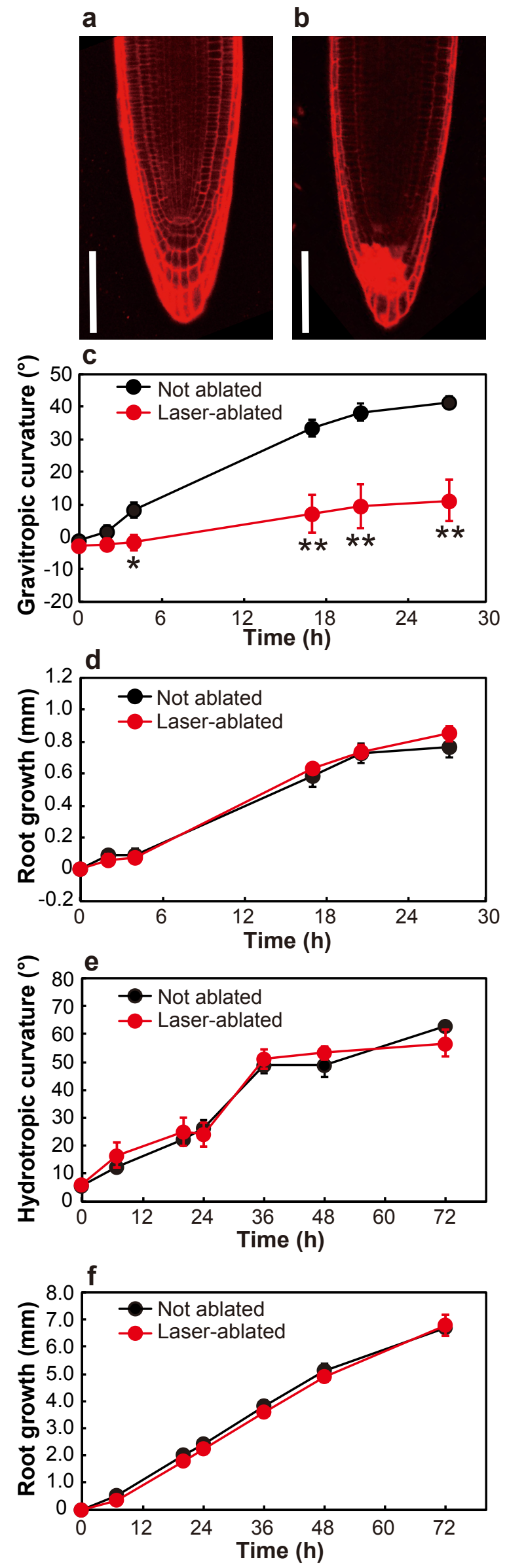
Figure 2

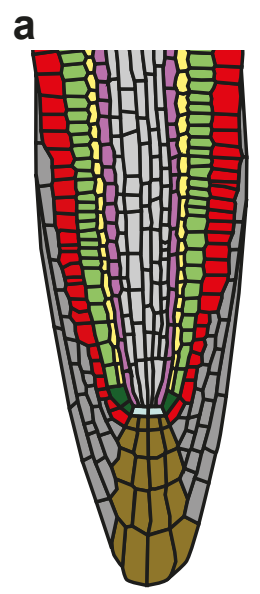

b

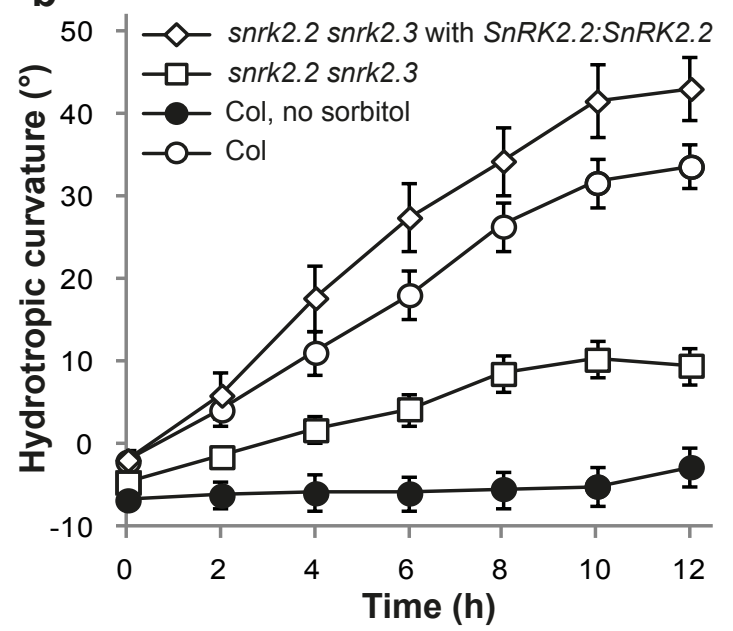

c
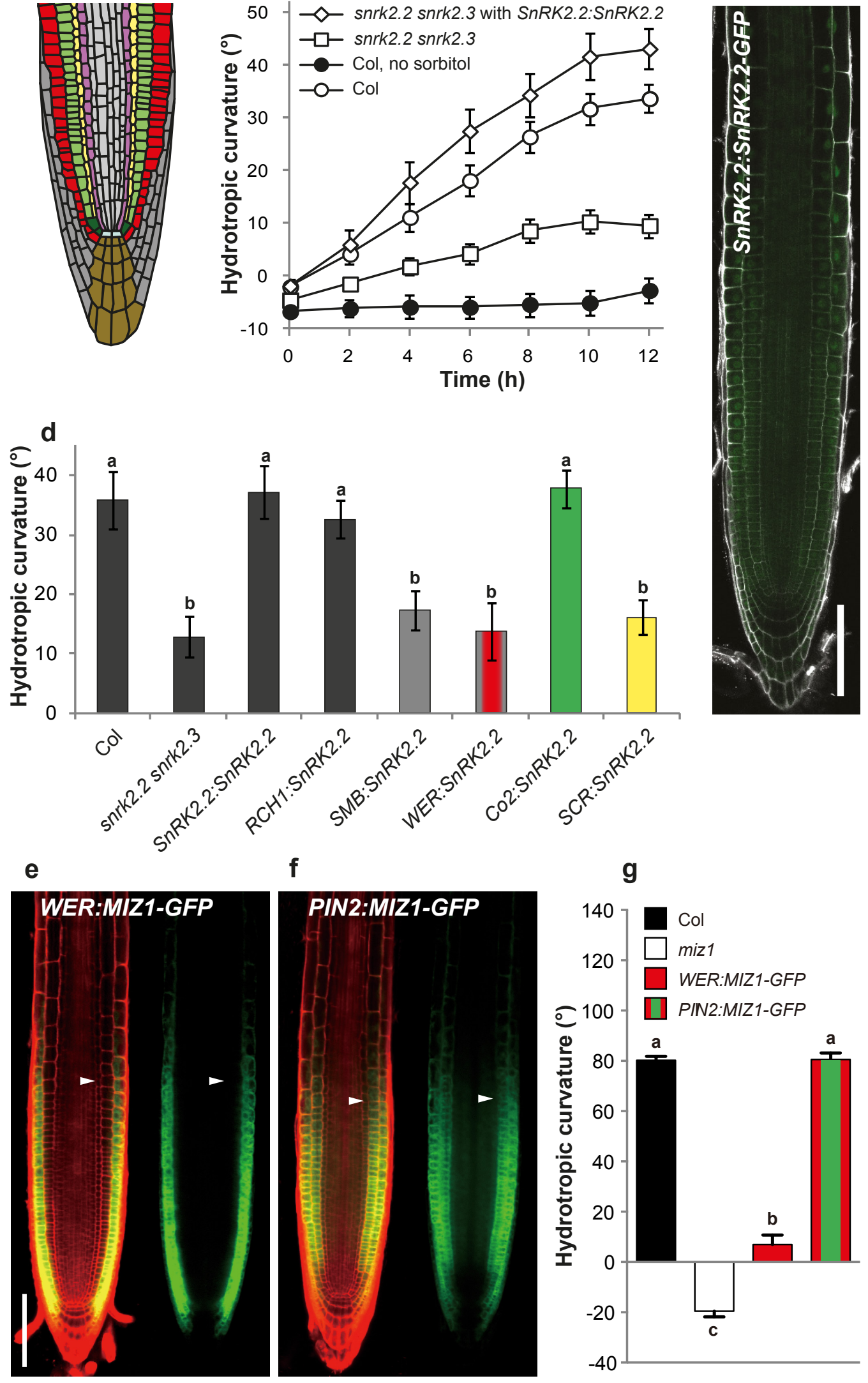
Figure 3
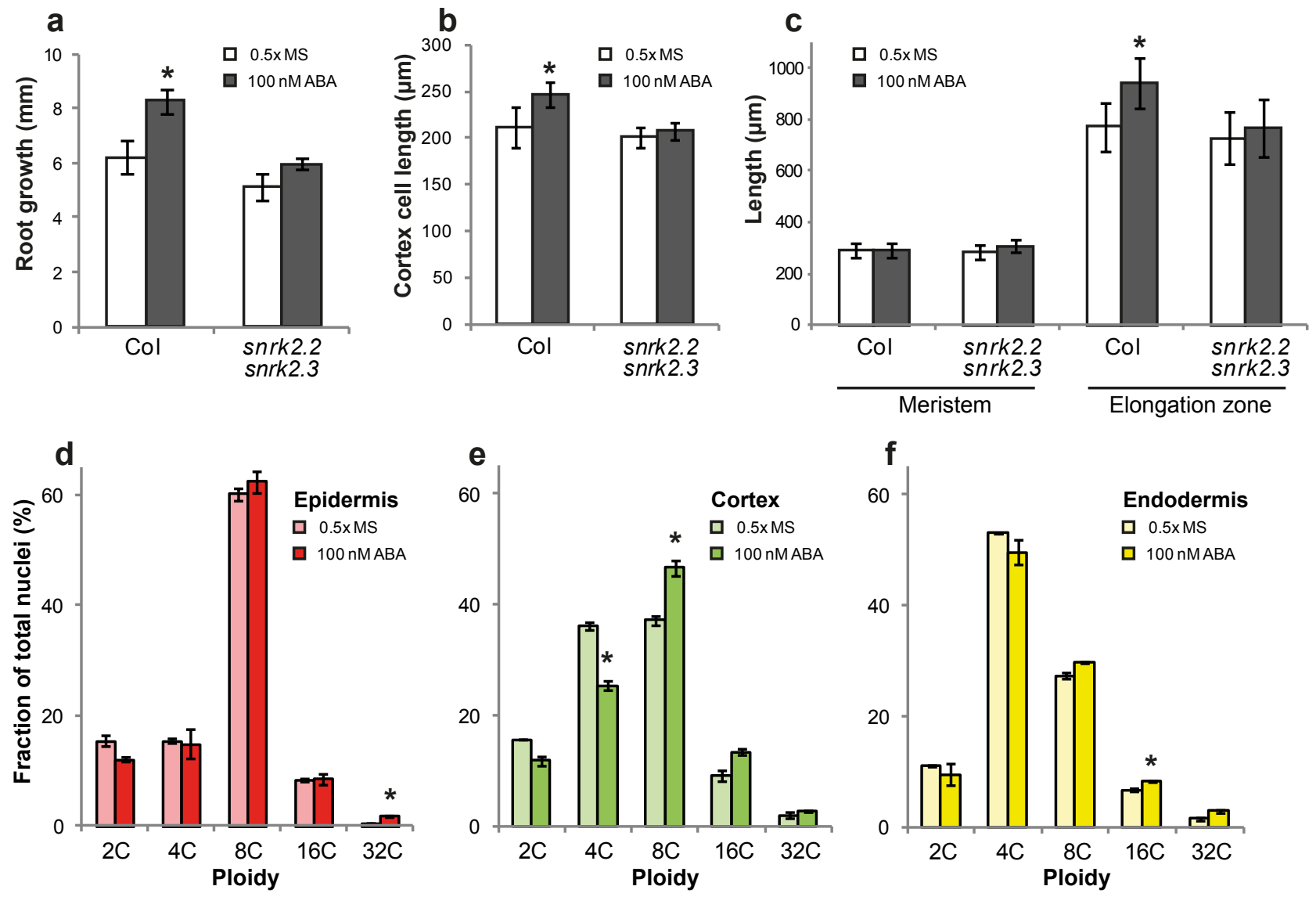
Figure 4
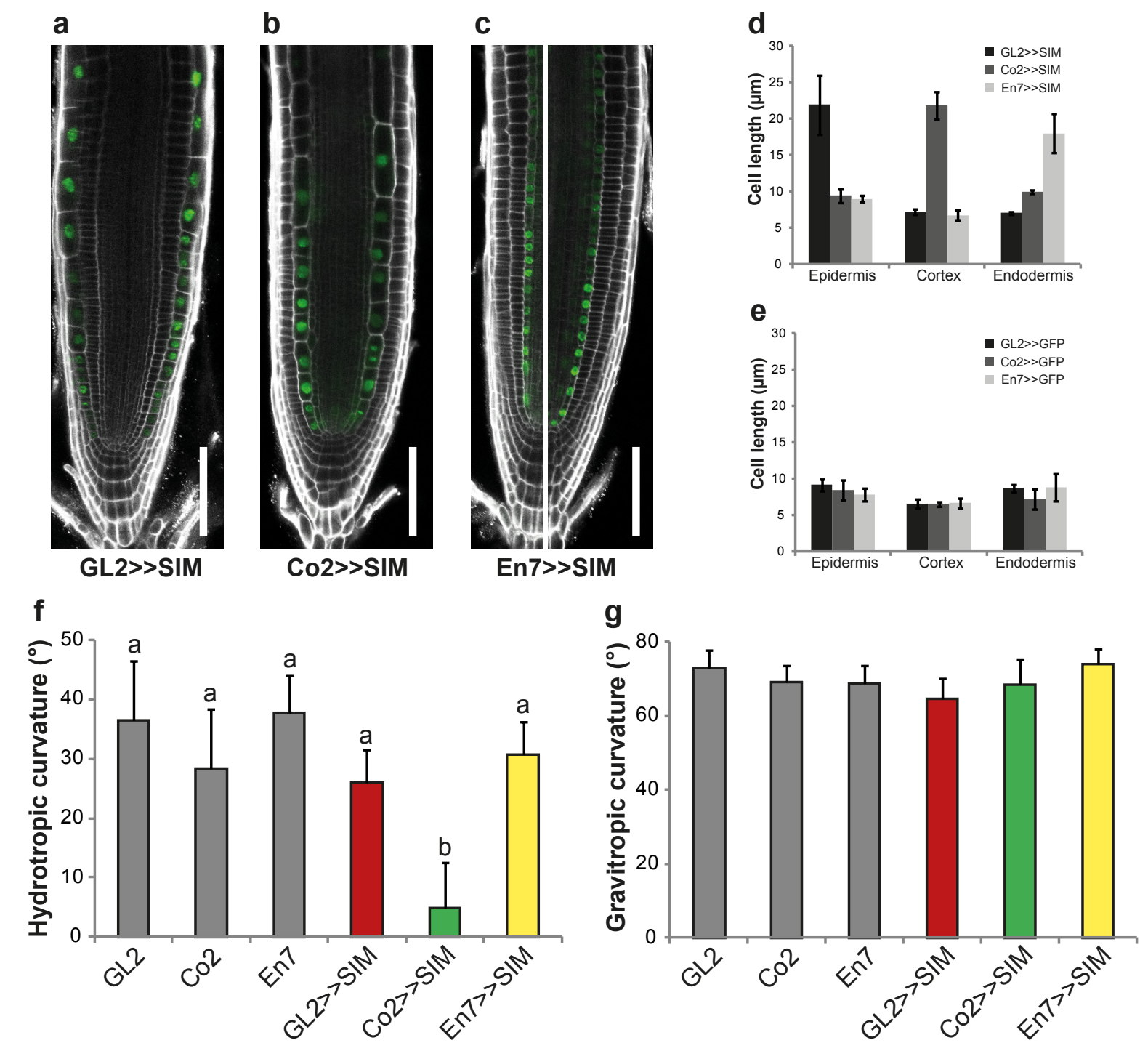
Figure 5

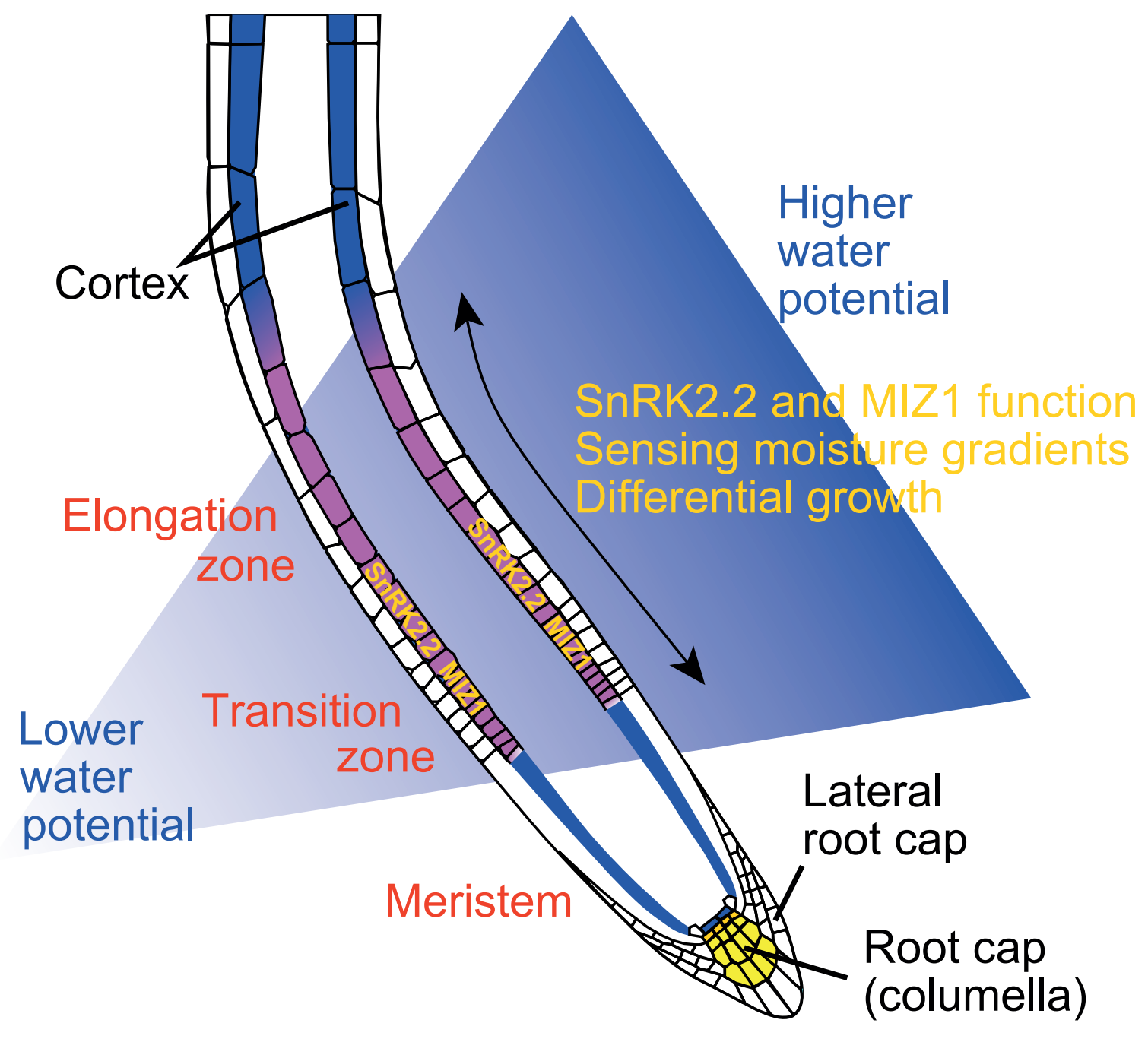


a

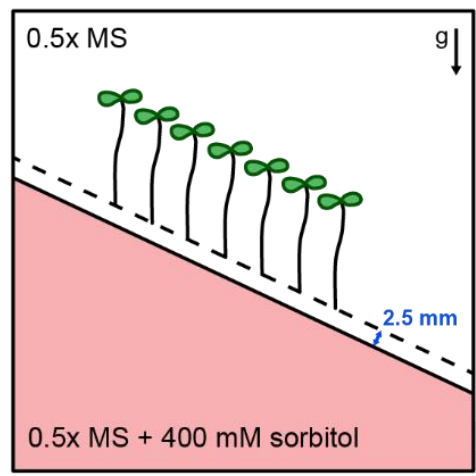

b

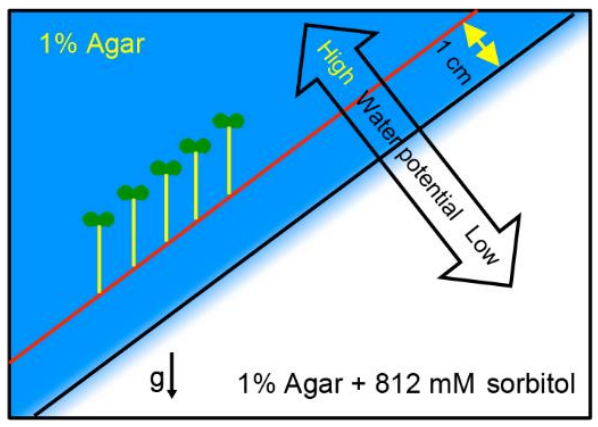

c

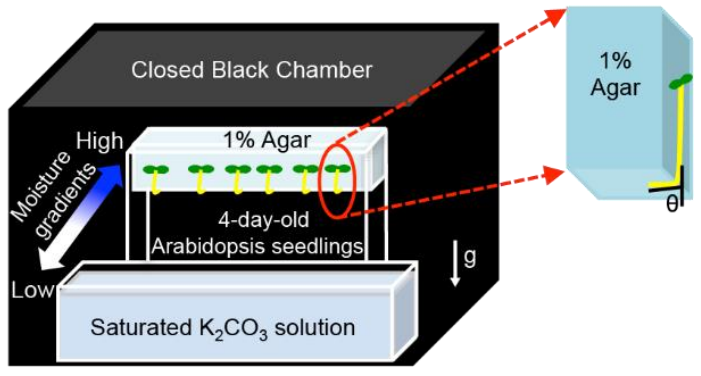

d

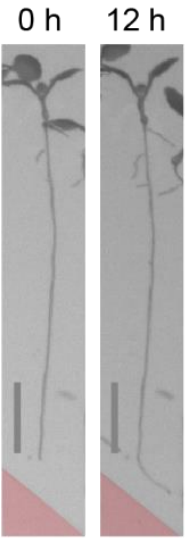

Col

f

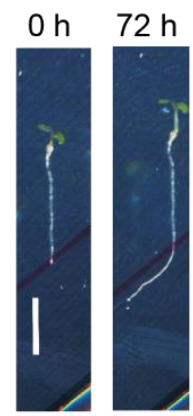

Col e
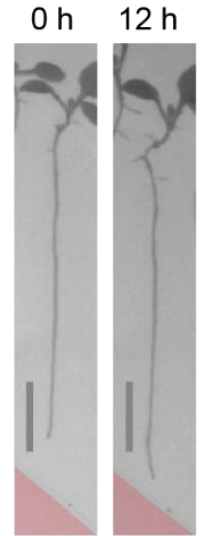

snrk2.2 snrk2.3

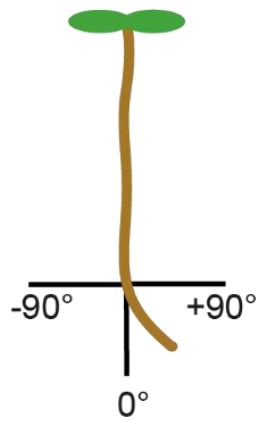

g

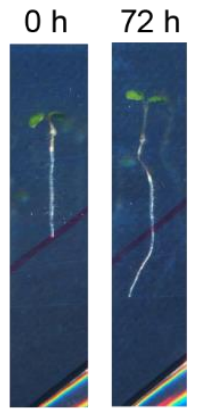

h

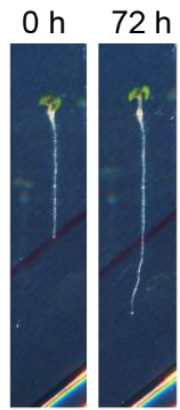

snrk2.2 snrk2.3

$\operatorname{miz1}$

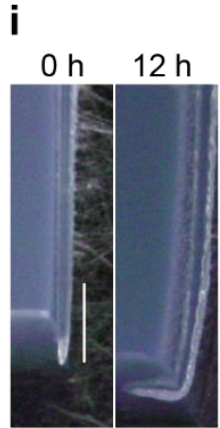

Col j

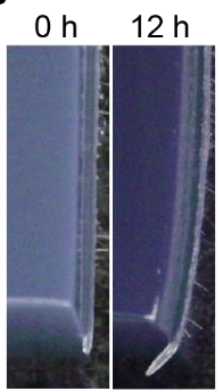

snrk2.2 snrk2.3 k

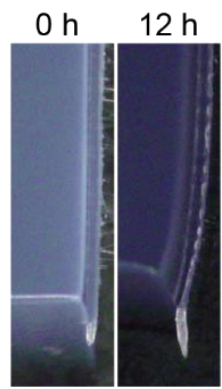

$\operatorname{miz1}$

\section{Supplementary Figure 1 Comparison of hydrotropism assays}

Hydrotropism assays were performed using a split-agar-based system with (a) 400 or (b) $812 \mathrm{mM}$ sorbitol or (c) a moisture gradient in air system. For detailed description of these assays see methods and references cited therein. $\mathbf{d}$, e Representative images of seedlings at the start $(0 \mathrm{~h})$ and end $(12 \mathrm{~h})$ of hydrotropism assays performed with split-agar plates using $400 \mathrm{mM}$ sorbitol, scale bars $=5 \mathrm{~mm}$. $\mathbf{f}-\mathbf{h}$ Representative images of seedlings at the start $(0 \mathrm{~h})$ and end $(72 \mathrm{~h})$ of hydrotropism assays performed with split-agar plates using 812 $\mathrm{mM}$ sorbitol, scale bars $=5 \mathrm{~mm}$. $\mathbf{i}$ - $\mathbf{k}$ Representative images of seedlings at the start $(0 \mathrm{~h})$ and end (12 h) of hydrotropism assays performed using a moisture gradient in air, scale bars $=1 \mathrm{~mm}$. Note that assays using $400 \mathrm{mM}$ sorbitol were performed in the UK while experiments using $812 \mathrm{mM}$ sorbitol and the moisture gradient in air were performed in Japan, with different growth rates of seedlings affecting the kinetics of hydrotropic bend. I In all assays bending of the root tip from the vertical $\left(0^{\circ}\right)$ towards medium with higher water potential was measured as a positive value. 
a
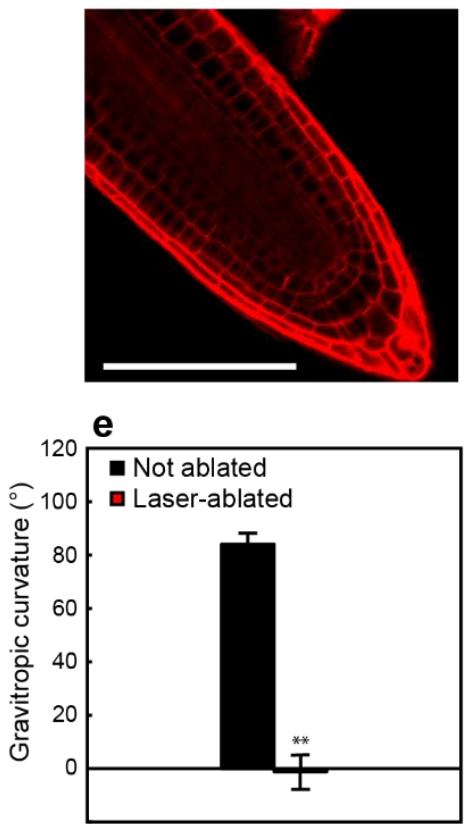

Columella

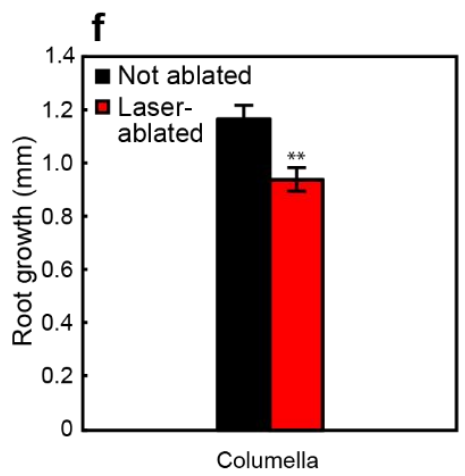

m b
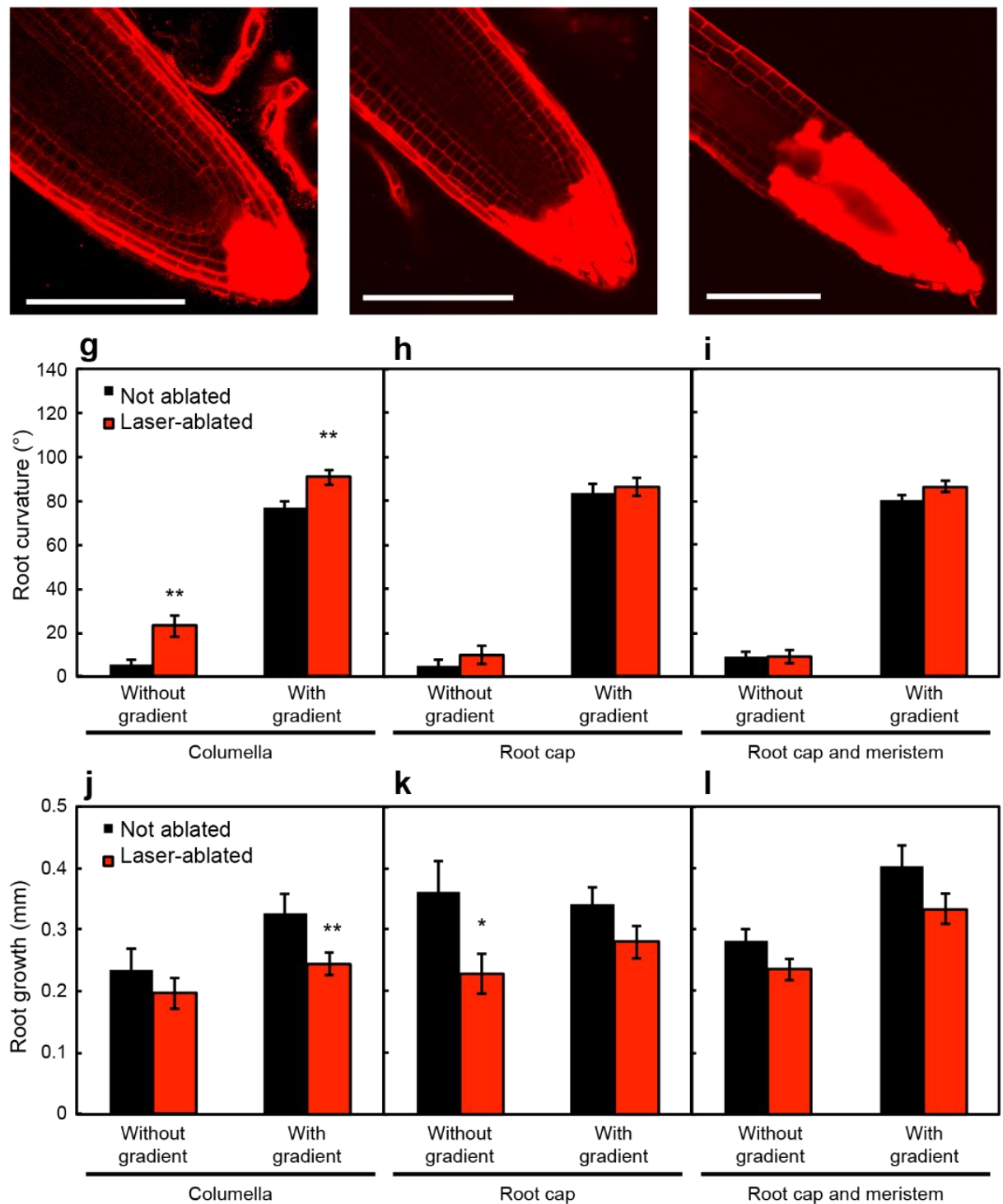

C

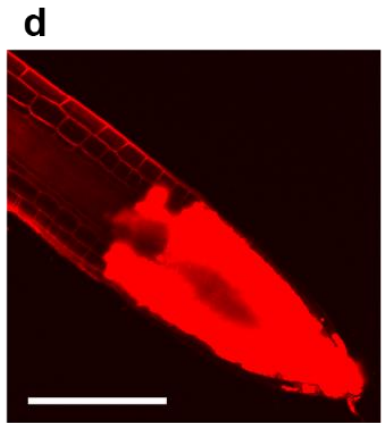

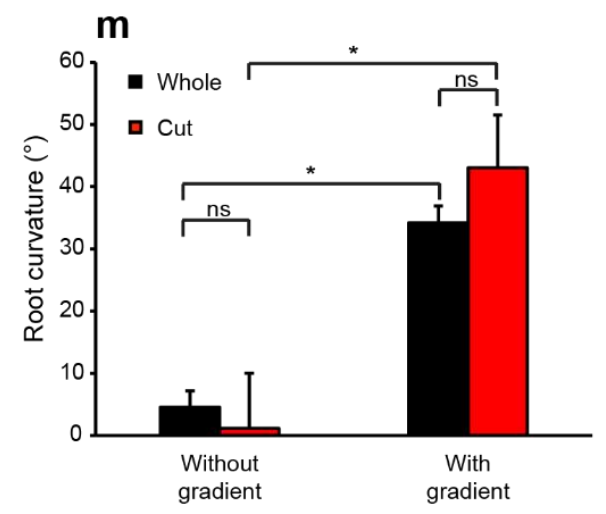

\section{Supplementary Figure 2 Effect of laser-beam ablation and microdissection of root-tip cells on gravitropic and hydrotropic responses}

Confocal fluorescence micrograph of propidium iodide (PI)-stained root cells before (a) and after (b - d) laser-beam ablation. Images of PI-stained cells show the ablation of (b) columella cells, (c) root-cap cells and (d) both the root cap and meristem, scale bars $=100 \mu \mathrm{m}$. Curvature (e) and elongation growth (f) $8 \mathrm{~h}$ after gravistimulation. Curvature $(\mathbf{g}-\mathbf{i})$ and elongation growth $(\mathbf{j}-\mathbf{I})$ in the absence or presence of a moisture gradient $8 \mathrm{~h}$ after ablation of $(\mathbf{g}, \mathbf{j})$ columella, $(\mathbf{h}, \mathbf{k})$ root cap and (i, I) both root cap and meristem. Hydrotropism assays were performed using the moisture gradient in air assay. Values are mean \pm SEM of three to six independent experiments, $n=24-62$. Asterisks indicate statistically significant differences $\left({ }^{*} p<0.05,{ }^{* *} p<0.01\right.$, Student's $t$-test). $\mathbf{m}$ Curvature $12 \mathrm{~h}$ after seedlings with whole or cut root tips were placed on hydrotropism plates without or with a water potential gradient. Hydrotropism assays were performed using the split-agar assay with $400 \mathrm{mM}$ sorbitol. $n=16-27$. Asterisks indicate statistically significant differences ( ${ }^{*} p<0.01$, Student's $t$-test, ns - not significant). 


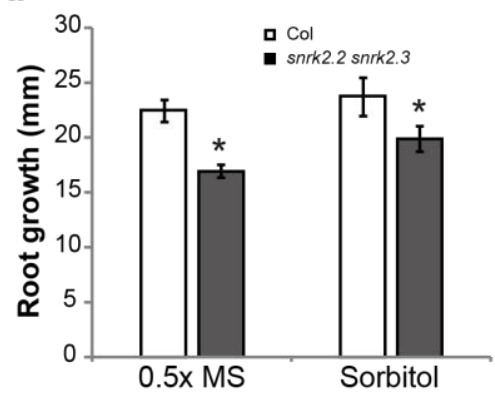

b

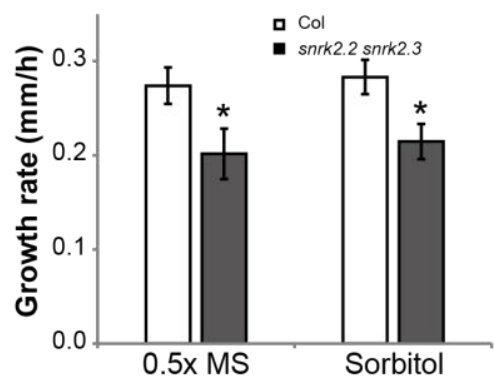

C

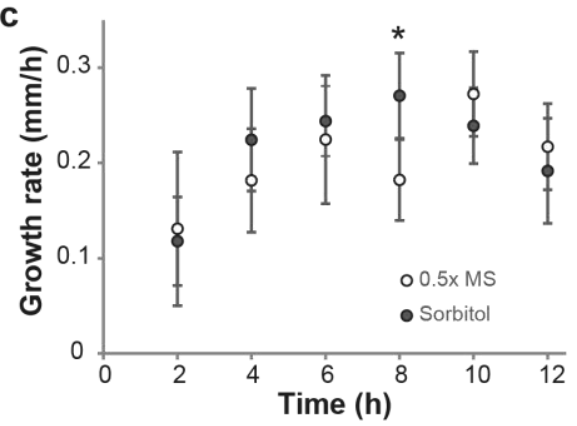

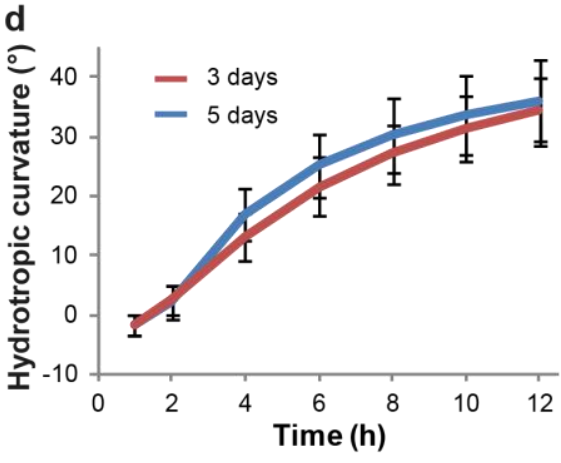

f

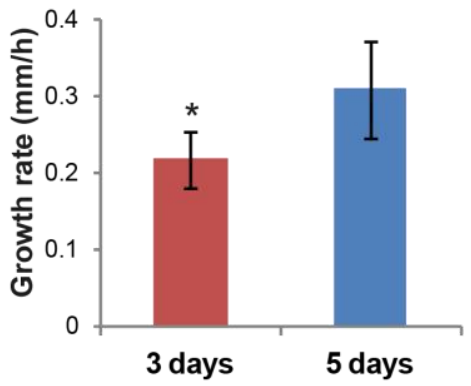

e

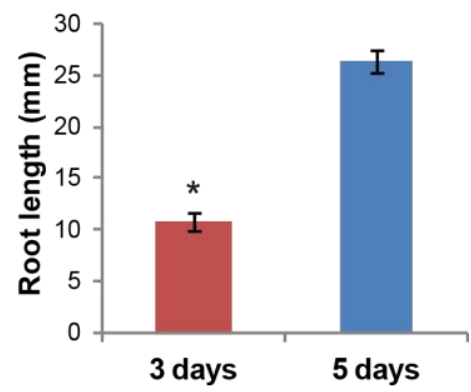

g

h

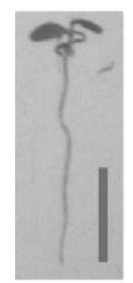

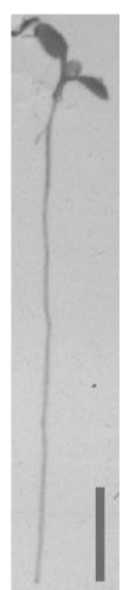

Supplementary Figure 3 Root tip angle of the hydrotropism response is not dependent on root growth rate

$\mathbf{a}-\mathbf{h}$ Relationship of hydrotropic bending to growth rate. All assays used split-agar system with $0.5 \mathrm{x}$ MS or $0.5 x$ MS plus $400 \mathrm{mM}$ sorbitol in the bottom half of the plate. a Root length at the start of the assay. Values are mean $\pm S D, n=20$. $\mathbf{b}$ Growth rate over the $12 \mathrm{~h}$ period of the assay. Values are mean $\pm 2 x$ SEM, $n=20$. c Kinetics of growth during the assay for snrk2.2 snrk2.3 seedlings. Values are mean $\pm 2 x$ SEM, $n=20$. d Hydrotropic curvature of 3 (red line) and 5 (blue line) day-old wild type roots after transfer of seedlings to split-agar plates with $400 \mathrm{mM}$ sorbitol. Values are mean $\pm 2 x \mathrm{SEM}, n=32-$ 36. e Root length of the same seedlings as in (d), measured at the start of the assay. Values are mean $\pm \mathrm{SD}, n=36$. $\mathbf{f}$ Growth rate of the same seedlings as in (d) over $12 \mathrm{~h}$ period of the assay. Values are mean \pm SD, $n=36 . \mathbf{g}-\mathbf{h}$ Images of (g) 3 and (h) 5 day-old seedlings at the start of the assay, scale bar $=5 \mathrm{~mm}$. Asterisks indicate statistically significant differences $\left({ }^{*} p<0.01\right.$, Student's $t$-test). 
a

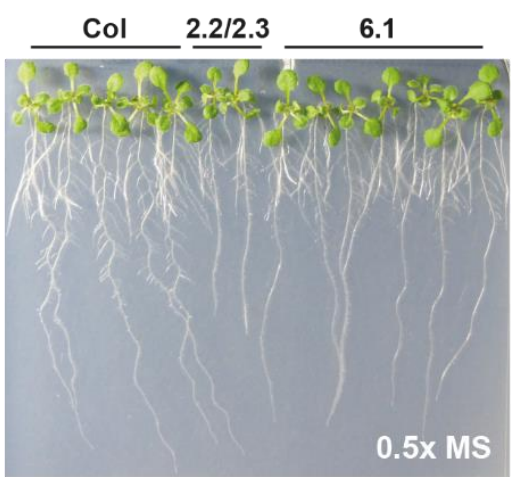

c

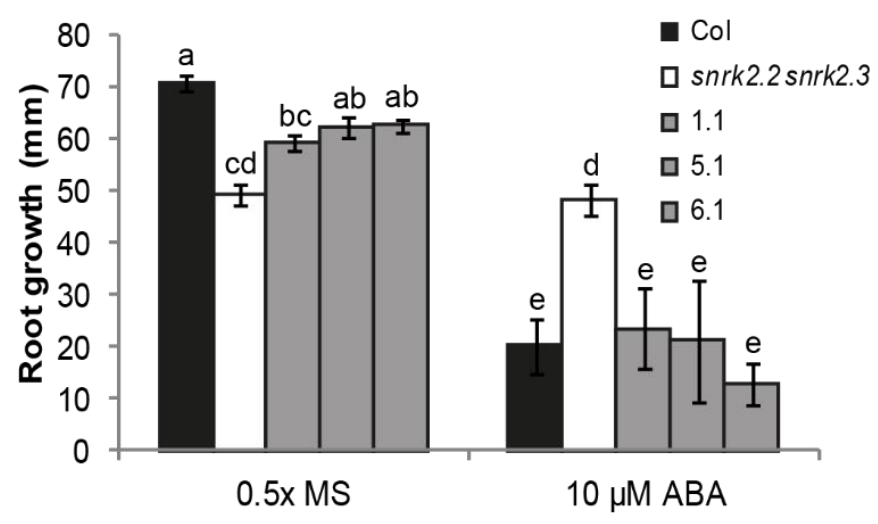

d

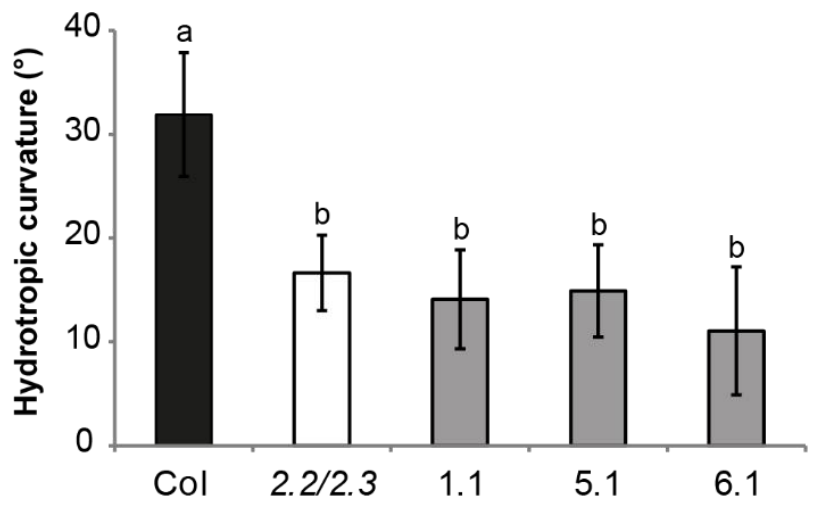

e
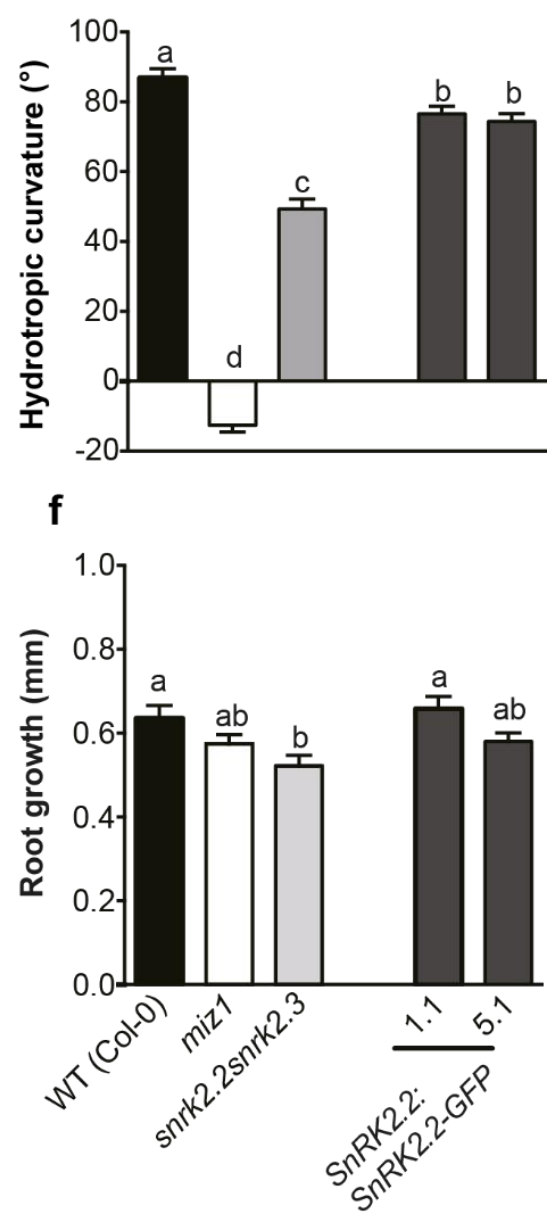

Supplementary Figure 4 The SnRK2.2-GFP fusion protein partially complements the ABA signalling defect of the snrk2.2 snrk2.3 mutant

a - c Root growth response to ABA. Images of Col, snrk2.2 snrk2.3 (2.2/2.3) and snrk2.2 snrk2.3 complemented with a SnRK2.2:SnRK2.2-GFP fusion (6.1) seedlings 6 days after transfer to plates (a) without and (b) with $10 \mu \mathrm{M}$ ABA. c Root growth of seedlings 6 days after transfer, three independent SnRK2.2:SnRK2.2-GFP lines (1.1, 5.1 and 6.1) were tested. Values are mean $\pm 2 x$ SEM, $n=7-28$. d Hydrotropic curvature on split-agar plates with $400 \mathrm{mM}$ sorbitol, mean $\pm 2 \times \mathrm{SEM}, n=54$. e Hydrotropic curvature and (f) root growth using the moisture gradient in air assay. Values are mean \pm SEM, $n=31-49$. Different letters in $\mathrm{c}-\mathrm{f}$ indicate statistically significant differences $(p<0.05$, Tukey HSD test). 

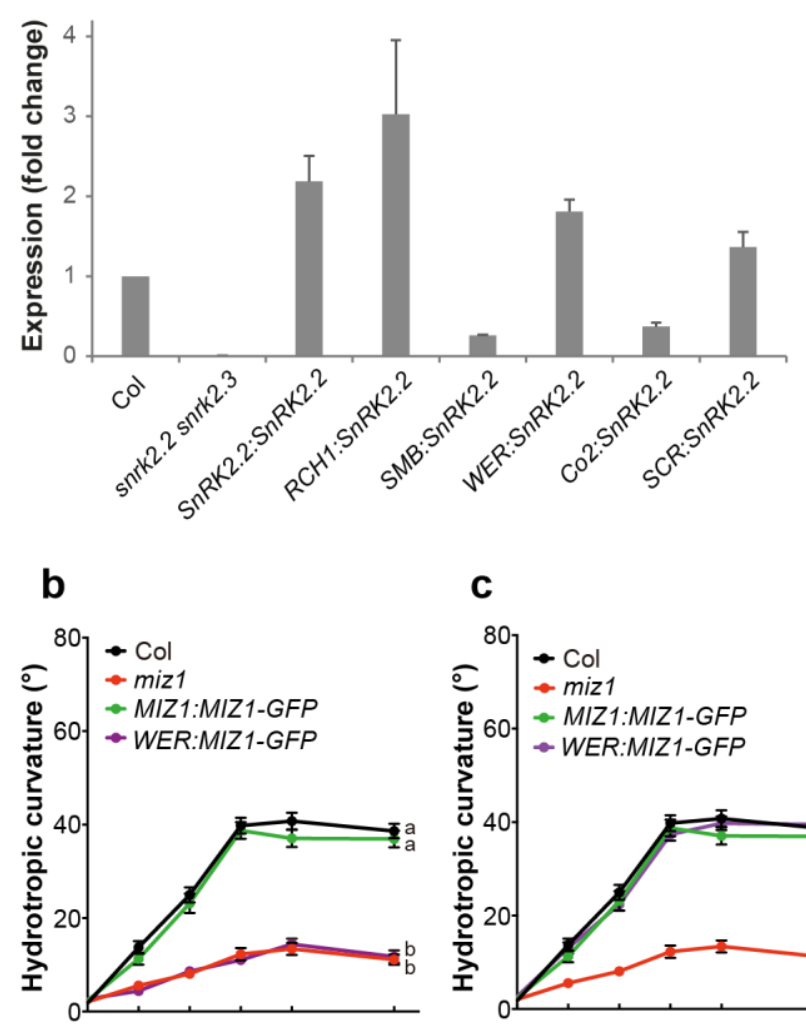

C
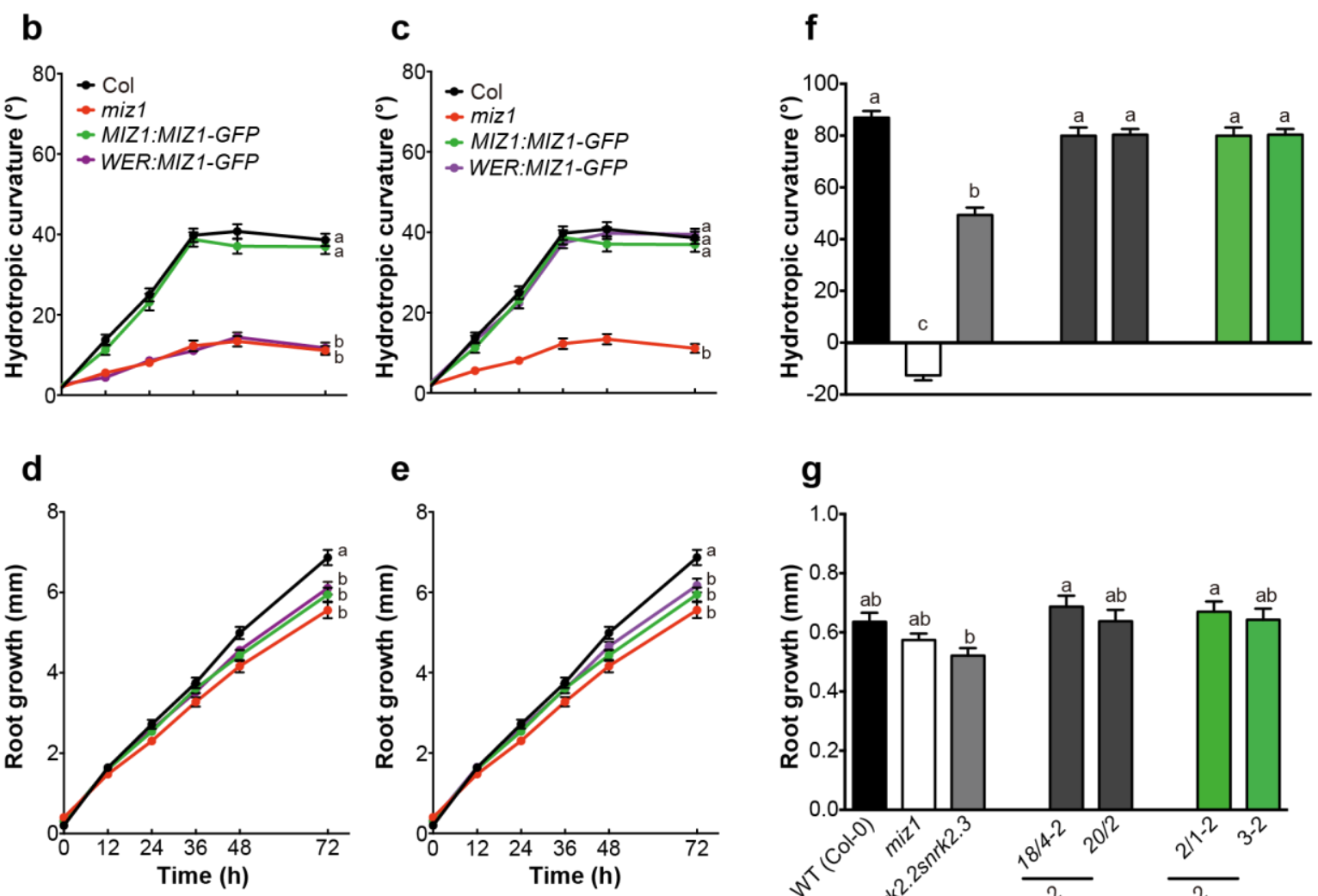

e

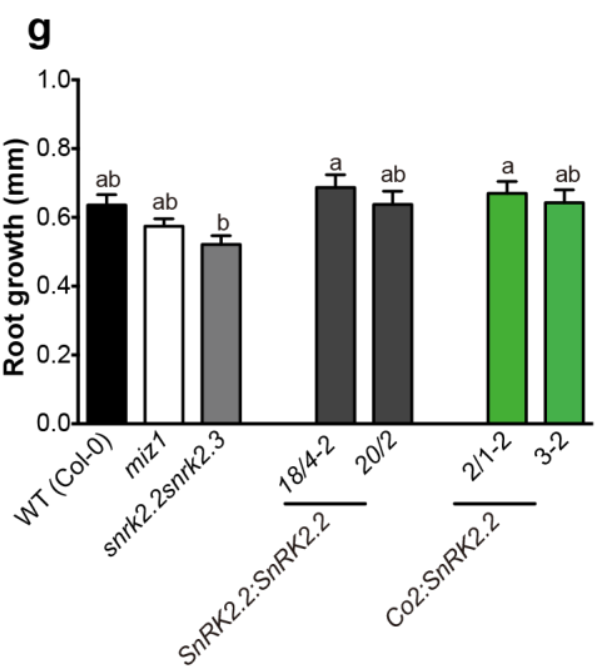

Supplementary Figure 5 SnRK2.2 expression in complementation lines and additional hydrotropism assays for complementing lines

a mRNA levels of SnRK2.2 in Col, snrk2.2 snrk2.3 and complementation lines quantified by RT-qPCR. Values are mean \pm SEM of three biological replicates and have been normalised to expression in Col. b, c Hydrotropic curvature and $\mathbf{d}$, e root growth of WER:MIZ1-GFP and PIN2:MIZ1-GFP complementation lines using the split-agar assay with $812 \mathrm{mM}$ sorbitol. Values are mean \pm SEM, $n=39$-56. Different letters indicate statistically significant differences at $72 \mathrm{~h}$ after hydrostimulation $(p<0.05$, Tukey HSD test). $\mathbf{f}$ Hydrotropic curvature and $\mathbf{g}$ root growth of SnRK2.2 and Co2 complementation lines $12 \mathrm{~h}$ after start of the moisture gradient in air assay. Values are mean $\pm \mathrm{SEM}, n=31$ 49. Different letters indicate statistically significant differences $(p<0.05$, Tukey HSD test). 


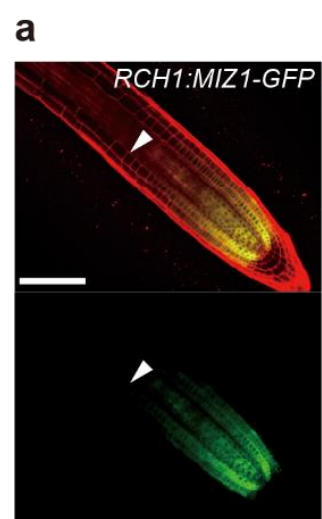

b

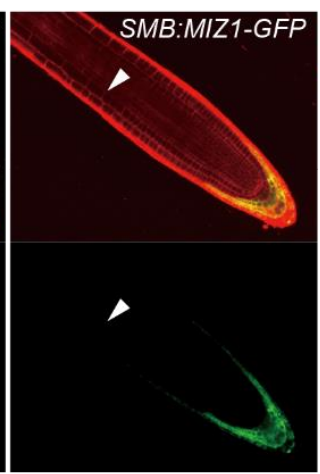

d

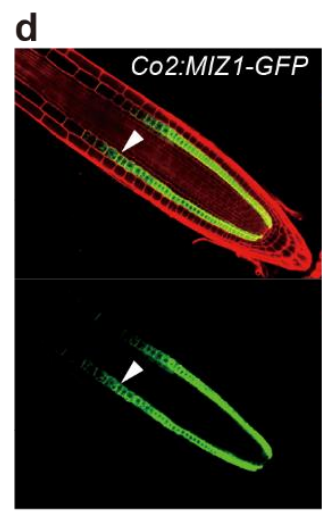

C

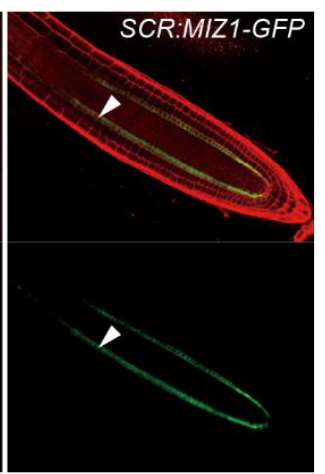

f

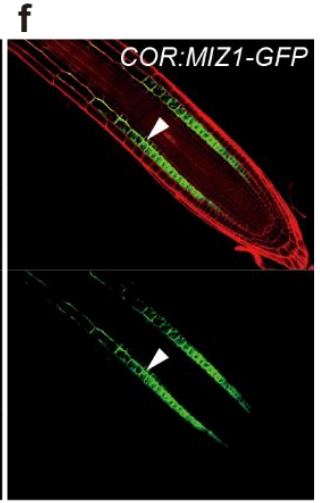

g
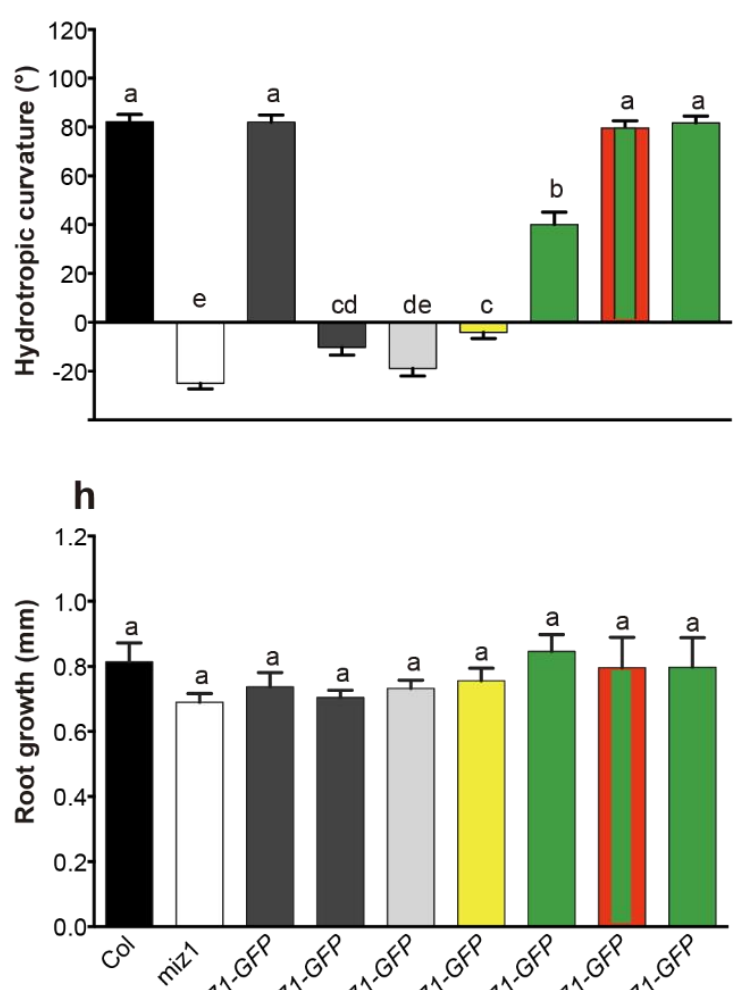

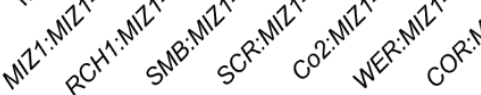

Supplementary Figure 6 MIZ1-GFP expression in the cortex is able to rescue the hydrotropic response of miz1 roots

a-f Confocal laser scanning microscope images of roots expressing MIZ1-GFP fusion protein under control of the (a) RCH1, (b) $S M B$, (c) $S C R$, (d) Co2, (e) WER and (f) COR promoters with MIZ1 terminator. Cell walls were stained with propidium iodide (red). Arrowhead indicates the approximate rootward boundary of the elongation zone, scale bar $=$ $100 \mu \mathrm{m}$. g Hydrotropic curvature and (h) root growth $12 \mathrm{~h}$ after start of the moisture gradients in air assay. Values are mean \pm SEM of three independent experiments, $n=29$ 110. Different letters indicate statistically significant differences $(p<0.05$, Tukey HSD test). 


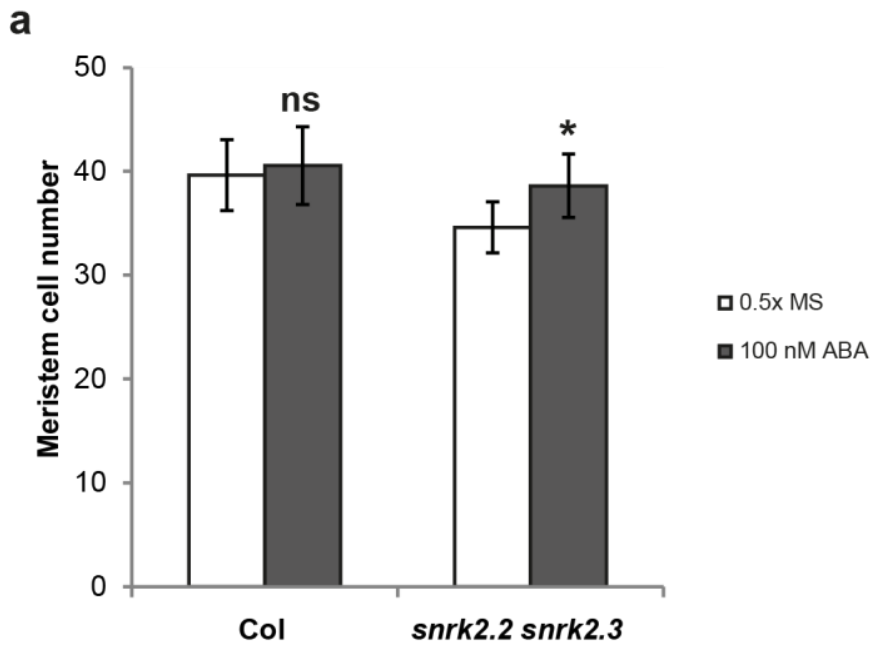

b

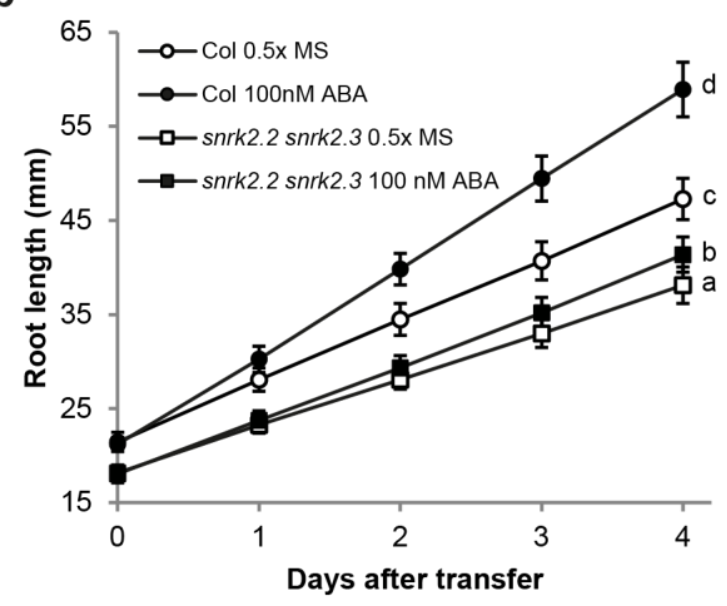

d

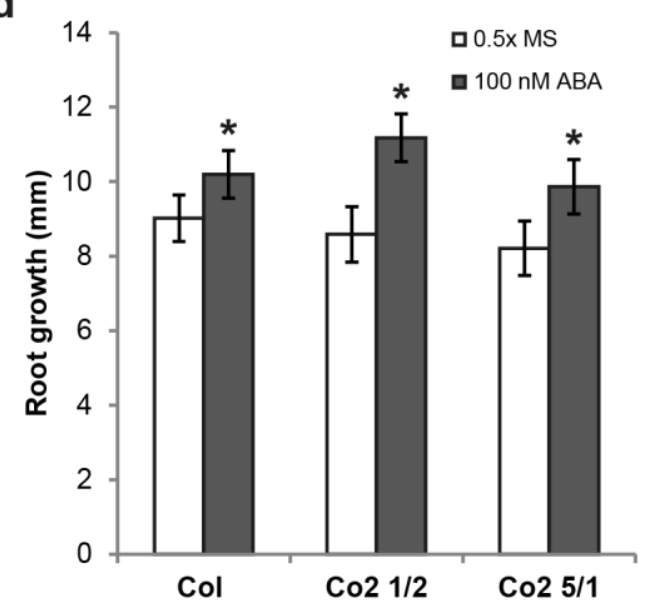

C

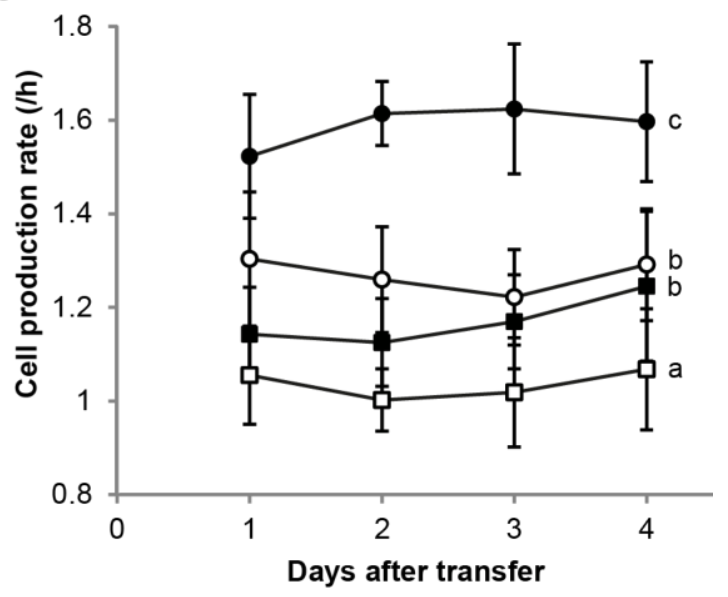

e

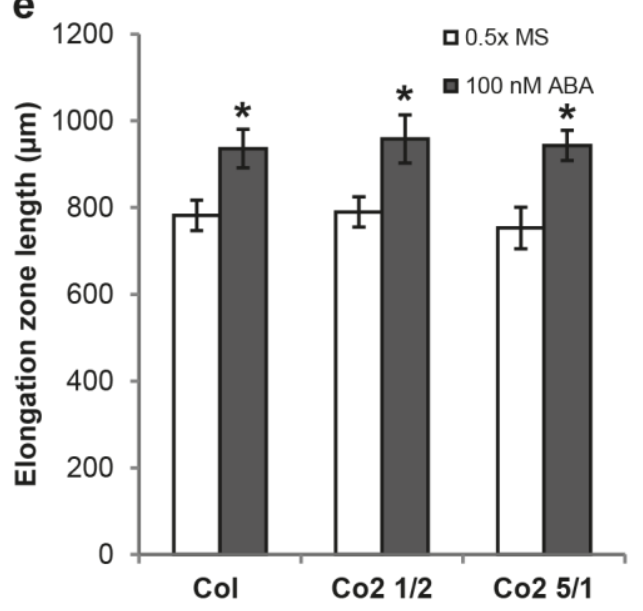

\section{Supplementary Figure 7 Effects of $100 \mathrm{nM}$ ABA on root growth}

a Meristem cell number $24 \mathrm{~h}$ after start of treatment without (light bars) and with $100 \mathrm{nM}$ ABA (grey bars). Values are mean $\pm \mathrm{SD}, n=10-28$. Asterisks indicate statistically significant differences ( ${ }^{*} p<0.01$, Student's $t$-test); ns not statistically significant. $\mathbf{b}$ Root length and (c) cell production rates in response to $100 \mathrm{nM} \mathrm{ABA}$. Values are mean $\pm \mathrm{SD}, n=$ 20. Different letters indicate statistically significant differences at 4 days after transfer $(p<$ 0.05 , Tukey HSD test). $\mathbf{d}$ Root growth and (e) elongation zone length in response to 100 nM ABA of Col and two independent Co2:SnRK2.2 lines. Values are (d) mean \pm SD, $n=15$ and (e) mean $\pm 2 x$ SEM, $n=10$. Asterisks indicate statistically significant differences $\left({ }^{*} p<\right.$ 0.01 , Student's $t$-test). 

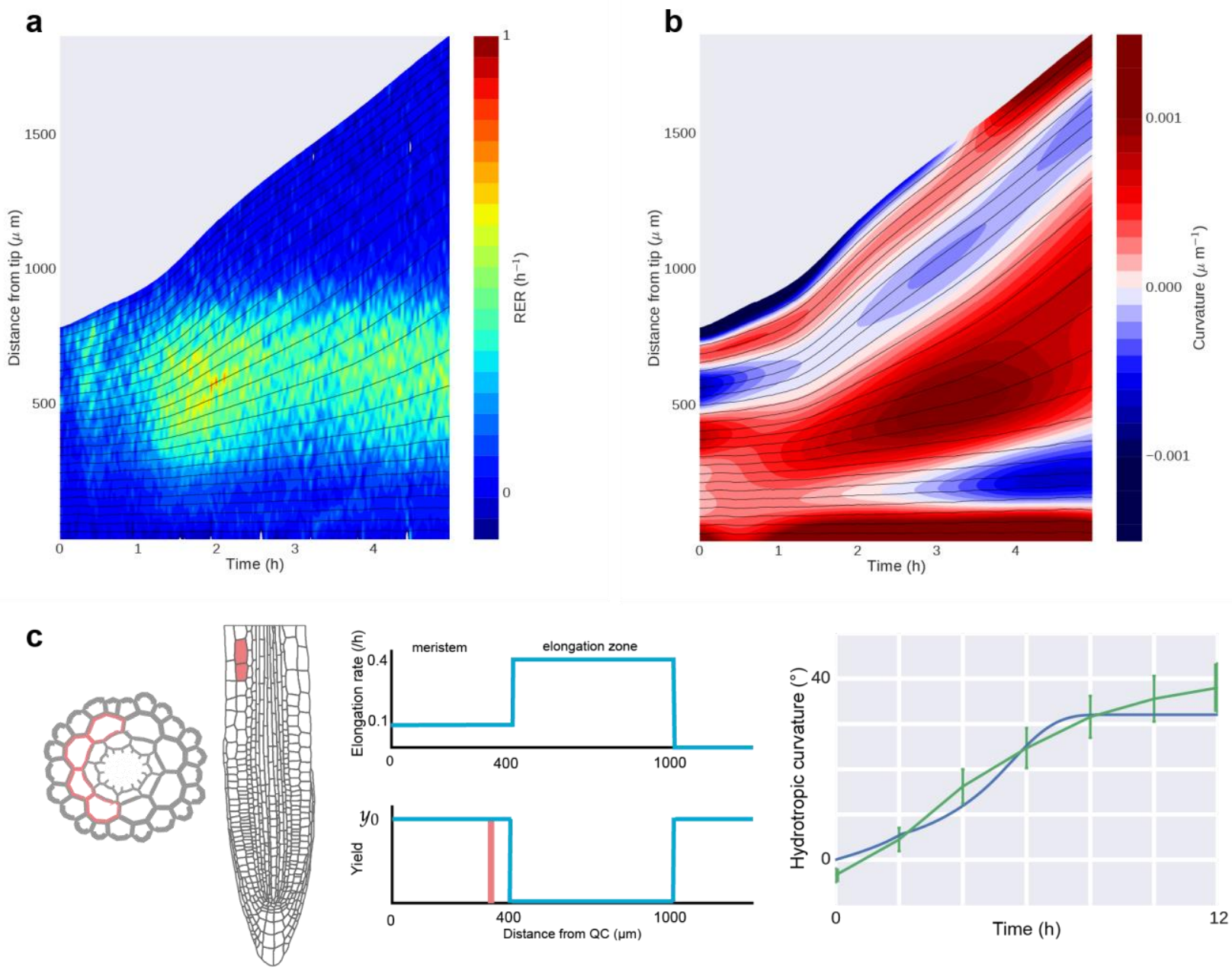

\section{Supplementary Figure 8 Changes in root growth in response to hydrotropism}

a Relative elongation rate and $\mathbf{b}$ curvature of a hydrotropically bending root during the first $5 \mathrm{~h}$ after transfer to a split-agar plate with $400 \mathrm{mM}$ sorbitol. Solid lines show the trajectories of points equally spaced at time zero. Representative data from four independent repeats shown. c Modelling the hydrotropism response: The transition between the meristem and elongation zone is marked by a drop in yield stress leading to a rise in elongation rate (centre); the large yield stress $y_{0}$ in the meristem inhibits cell expansion; cortical cells on the dry side of the root (pink in transverse and axial cross-sections, left) enter elongation early for the first $2 \mathrm{~h}$, with asymmetric softening across the root generating a bend. Simulated (blue) and experimental (green) hydrotropic curvature profiles are compared (right). 


\section{Supplementary Methods}

\section{Plant material and growth conditions}

The accession used for all experiments in this study is Arabidopsis thaliana Columbia-0 (Col0). Plants were grown in a controlled environment room $\left(22^{\circ} \mathrm{C} / 18^{\circ} \mathrm{C}\right)$, with a $16 \mathrm{~h}$ light $8 \mathrm{~h}$ dark photoperiod regime at $150 \mu \mathrm{mol} \mathrm{m} \mathrm{m}^{-2} \mathrm{~s}^{-1}$ in compost (M3, Levington). For detailed root growth assays, seeds were surface sterilized with $0.25 \%-50 \%(\mathrm{v} / \mathrm{v})$ sodium hypochlorite and $0.01 \%(\mathrm{v} / \mathrm{v})$ Triton X-100, washed three times with sterile water and sown on plates with $0.5 x$ Murashige and Skoog (MS) medium (Sigma) with or without $0.4 \%(w / v)$ sucrose and solidified with either $0.3 \%(\mathrm{w} / \mathrm{v})$ gellan gum (Sigma) or $1 \%(\mathrm{w} / \mathrm{v})$ Bactoagar (Difco). After two days at $4^{\circ} \mathrm{C}$ in the dark, plates were placed vertically in a growth room at $22-23^{\circ} \mathrm{C}$ with continuous light at $100-150 \mu \mathrm{mol} \mathrm{m}^{-2} \mathrm{~s}^{-1}$.

\section{Complementation of snrk2.2 snrk2.3}

To construct the SnRK2.2:SnRK2.2-GFP transgene, a fragment comprising $2 \mathrm{~kb}$ upstream of the ATG start codon plus the SnRK2.2 coding sequence was amplified from A. thaliana genomic DNA by PCR, cloned into pCR8/GW/TOPO TA (Life Technologies) and verified by sequencing. Next, it was recombined by Gateway LR reaction into pGWB404 destination vector ${ }^{1}$.

For tissue-specific complementation of the snrk2.2 snrk2.3 mutant the coding sequence (including introns) of SnRK2.2 was amplified from $A$. thaliana genomic DNA by PCR (Phusion, New England Biolabs) with an Xhol site at the 5' and a Pstl site at the 3' end. The promoter sequences of $S n R K 2.2, R C H 1^{2}, S M B^{3}$ and $W E R^{4}$ were amplified with a Kpnl at the 5' and an Xhol site at the 3' end. PCR products were ligated into the pGEM-T Easy vector (Promega) and verified by sequencing. The coding sequence of SnRK2.2 was cut with Xhol and Pstl from pGEM-T Easy and ligated into the same sites of the vector pG0229-T ${ }^{5}$. The resulting vector was then opened with $\mathrm{Kpnl}$ and Xhol and ligated with the SnRK2.2, $R C H 1$, $S M B$ or WER promoter sequences which had been excised from pGEM-T Easy with the same enzymes. The $C o 2^{6}$ and $S C R^{7}$ promoters were excised with $\mathrm{Pstl} / \mathrm{Kpnl}$ and Sall/BamHI from vectors $\mathrm{BJ} 36-\mathrm{pCo} 2^{6}$ and $\mathrm{pE} 11 \mathrm{SCR}: \mathrm{GAI}$ (gai):YFP ${ }^{8}$ respectively, made blunt with $\mathrm{T} 4$ DNA polymerase and then ligated into the Xhol site of pG0229-T containing the SnRK2.2 coding sequence.

Constructs were transferred to $A$. tumefaciens GV3101 pMP90 by electroporation and used to transform snrk2.2 snrk2.3 plants by floral dipping as described previously ${ }^{9}$. At least two independent homozygous $\mathrm{T}_{3}$ lines were used for root growth and hydrotropism assays. 


\section{Complementation of miz1}

The sequences of $R C H 1, S M B, S C R, W E R, C O R$, and Co2 promoters were amplified from the pG0229-T vectors described above with forward and reverse primers containing Acc65I and Eagl restriction sites, respectively (Supplementary Table 1). For constructs with tissuespecific promoters, the MIZ1 promoter region of a MIZ1:MIZ1-GFP construct previously reported $^{10}$ was replaced with those tissue-specific promoters. For constructing WER:MIZ1GFP (-HSPter) and PIN2:MIZ1-GFP (-HSPter), three DNA fragments, namely promoter regions of WER and PIN2 as well as coding region of MIZ1-GFP, were PCR-amplified and then cloned into pRI201 vector using In-Fusion Cloning kit (Clontech). All constructs were introduced into A. tumefaciens (strain GV3101 or LBA4404) by freeze/thaw shock method and transformed into miz1 mutants with the floral dip method. Selection of transformed plants was performed on the half-strength MS medium with $0.4 \%$ sucrose supplemented with $50 \mu \mathrm{g} / \mathrm{mL}$ kanamycin, $100 \mu \mathrm{g} / \mathrm{mL}$ carbenicillin and $10 \mu \mathrm{g} / \mathrm{mL}$ benomyl. PCR amplification method was adopted to confirm the presence of transgenes.

\section{Tissue-specific overexpression of SIAMESE}

A GAL4-VP16 driven transactivation system ${ }^{11}$ was used to co-express SIM and a nuclearlocalised GFP marker specifically in either epidermis, cortex, or endodermis. The pUAS-SIM construct was obtained by multisite Gateway cloning combining pDONR221-SIM (kindly provided by John Larkin ${ }^{12}$ ), pEN-L4-UAS-R1 (gateway.psb.ugent.be) and pH7m24GW, $3^{13}$. For the endodermis, cortex and atrichoblast marker lines the promoters of En7, Co2 and GL2 were cloned ${ }^{6,14}$. A 1220bp En7, 550bp Co2 and 2066bp GL2 promoter fragment was PCR amplified, cloned into the pDONR-P4-P1R vector and verified by sequencing. The latter vectors were recombined with pK9-H2AF-UAS-7m24GW (gateway.psb.ugent.be) and pDONR-L1-GAL4-VP16-R2 (kindly provided by Wim Grunewald) to obtain pEn7-GAL4pUAS-H2AF, pCo2-GAL4-pUAS-H2AF and pGL2-GAL4—pUAS-H2AF respectively. The obtained vectors were transferred to $A$. tumefaciens strain $\mathrm{C} 58 \mathrm{C} 1 \mathrm{pMP} 90$, which was used to transform $A$. thaliana Col-0.

The En7>>SIM, Co2>>SIM and GL2>>SIM lines were generated by pollinating homozygous pUAS-SIM lines with homozygous lines bearing the pEn7-GAL4-pUAS-H2AF, pCo2GAL4-pUAS-H2AF and pGL2-GAL4-pUAS-H2AF constructs. Two independent pUASSIM lines were used for crossing and all experiments were performed with $F_{1}$ seeds from these crosses.

\section{Confocal laser scanning microscopy}

Cell walls of root tips were stained with $5 \mu \mathrm{g} / \mathrm{mL}$ propidium iodide (Molecular Probes, Sigma). Images were taken with a Leica SP5 microscope (Leica Microsystems Ltd) or 
Olympus FV-1000 (Olympus) using the $488 \mathrm{~nm}$ laser for excitation and collecting emitted light at 500-550 nm (GFP) and 610-665 nm (propidium iodide).

\section{Quantitative RT-PCR}

Total RNA was extracted from 5 day-old excised root tips (root tip to first visible root hair bulge, $n=100$ ) using the RNeasy Plus Micro kit with genomic DNA eliminator columns (Qiagen). Poly(dT) cDNA was prepared from total RNA using the RevertAid first strand cDNA synthesis kit (Thermo Scientific). Quantitative PCR was performed using SYBR Green Sensimix (Bioline) on a LightCycler 480 machine (Roche). Quantification of SnRK2.2 expression was performed with primers specific to the 3' region of the gene, with expression levels normalised to the ubiquitin-associated gene UBA (At1g04850). All primer sequences can be found in supplementary table 1 .

\section{Ploidy measurements of root tissues}

Tissue-specific ploidy measurements were obtained via flow cytometric analysis of nucleartagged Arabidopsis thaliana GFP marker lines.

24 hours after transfer of five-day-old plants to $100 \mathrm{nM} \mathrm{ABA}$ and MS standard media, root tips of approximately $0.5 \mathrm{~cm}$ length were excised and used for nuclear extraction. Root tips were chopped with a razor blade in $200 \mu \mathrm{L}$ of nuclei extraction buffer ${ }^{15}$ containing $45 \mathrm{mM}$ $\mathrm{MgCl}_{2}, 30 \mathrm{mM}$ sodium citrate, and $20 \mathrm{mM}$ 3- morpholinopropane-1-sulfonic acid, $\mathrm{pH} 7.0$ for 2 min, then filtered through a $50-\mu \mathrm{m}$ nylon filter. The DNA was stained with $1 \mathrm{mg} / \mathrm{ml}$ DAPI (4',6-diamidino-2-phenylindole $)^{16}$. Nuclei were measured using a CyFlow Flow Cytometer (Partec) excited by illumination at $395 \mathrm{~nm}$, and equipped with an additional $488 \mathrm{~nm}$ laser to excite and detect GFP-specific fluorescence. The DNA content of cells was derived from DAPI fluorescence measurements using FloMax software (Partec).

\section{Microdissection of root tips}

24 hours before hydrotropism assays were performed, four-day-old plants were transferred to sterile $100 \mu \mathrm{m}$ nylon mesh (Clarcor) on $0.5 \mathrm{x}$ MS plates to facilitate root dissection. 1 hour before transfer to hydrotropism assay plates, approximately $250 \mu \mathrm{m}$ of the distal region of the root tip were excised manually. After transfer to mesh and excision, plates were returned to the controlled environment room for recovery.

\section{Statistical analysis}

Statistical analysis of data was performed using Excel (Microsoft), KaleidaGraph (Synergy Software) and GenStat (VSN International). 


\section{Measuring root bending kinetics}

More detailed measurements of root bending kinetics were obtained using a pipeline which extended upon existing methods ${ }^{17-22}$. Roots were imaged on hydrotropism assay plates using an adapted brightfield microscope (Zeiss AxioStar Plus, Carl Zeiss Ltd.) as detailed previously ${ }^{23}$. Time-lapse images were acquired under near-infrared illumination using a machine vision camera connected to the microscope (Stingray F-504B, Allied Vision Technologies $\mathrm{GmbH}$ ). Images were taken at 2 minute intervals for 5 hours using custom software written using LabVIEW System Design Software (National Instruments Corporation). To estimate root motion, the optical flow field between successive image frames was generated by the DeepFlow algorithm ${ }^{24}$. In each frame, roots were separated from the background using a level set method ${ }^{25}$, some parts of which were adapted from other work (https://code.google.com/archive/p/ofeli/) ${ }^{26}$. After eliminating all background noise, root hairs were removed using a "rolling ball" algorithm ${ }^{27}$, and root boundaries were smoothed by the opening operation. Root midlines for each frame were obtained by calculating the longest path between two end nodes through the skeleton obtained from the medial axis transform. Further details of these steps can be found in Supplementary Note 2. To measure distances from a biologically relevant landmark, the approximate position of the root quiescent centre (QC) was manually specified in the first frame of the image sequence, and then tracked through the rest of the image sequence using the optical flow velocity fields. Midlines from skeletonization were adjusted to end at the QC in each frame. Relative motion between camera and plate was determined using the average of the optical flow field over a section of the image away from the root, and used to stabilize the midline profiles. Smoothed midline profiles were obtained using bivariate spline interpolation of the y-pixel positions over all frames ${ }^{28}$. Curvature and distance along the midline were calculated from these smoothed midlines; relative elongation rates at points along the midline were calculated using

$$
R E R=\boldsymbol{t} \cdot \frac{1}{2}\left(\nabla \boldsymbol{u}+\nabla \boldsymbol{u}^{T}\right) \boldsymbol{t}
$$

where $\boldsymbol{t}$ is the local tangent to the midline, and $\nabla \boldsymbol{u}$ the gradient of the vector flow velocity field at that point.

\section{Code availability}

All computer code generated for this study is freely available at github.

https://github.com/tuanthng/RootAnalysis

https://github.com/jfozard/hydrotopism-estimates 


\section{References}

$1 \quad$ Nakagawa, T. et al. Improved Gateway binary vectors: high-performance vectors for creation of fusion constructs in transgenic analysis of plants. Bioscience, Biotechnology, and Biochemistry 71, 2095-2100, doi:10.1271/bbb.70216 (2007). Casamitjana-Martinez, E. et al. Root-specific CLE19 overexpression and the sol1/2 suppressors implicate a CLV-like pathway in the control of Arabidopsis root meristem maintenance. Current Biology : CB 13, 1435-1441 (2003).

3 Willemsen, V. et al. The NAC domain transcription factors FEZ and SOMBRERO control the orientation of cell division plane in Arabidopsis root stem cells. Developmental Cell 15, 913-922, doi:10.1016/j.devcel.2008.09.019 (2008). Lee, M. M. \& Schiefelbein, J. WEREWOLF, a MYB-related protein in Arabidopsis, is a position-dependent regulator of epidermal cell patterning. Cell 99, 473-483 (1999).

Footitt, S. et al. The COMATOSE ATP-binding cassette transporter is required for full fertility in Arabidopsis. Plant Physiol 144, 1467-1480, doi:10.1104/pp.107.099903 (2007).

Heidstra, R., Welch, D. \& Scheres, B. Mosaic analyses using marked activation and deletion clones dissect Arabidopsis SCARECROW action in asymmetric cell division. Genes \& Development 18, 1964-1969, doi:10.1101/gad.305504 (2004). Wysocka-Diller, J. W., Helariutta, Y., Fukaki, H., Malamy, J. E. \& Benfey, P. N. Molecular analysis of SCARECROW function reveals a radial patterning mechanism common to root and shoot. Development 127, 595-603 (2000). Ubeda-Tomas, S. et al. Root growth in Arabidopsis requires gibberellin/DELLA signalling in the endodermis. Nature Cell Biology 10, 625-628, doi:10.1038/ncb1726 (2008).

9 Dietrich, D. et al. Mutations in the Arabidopsis peroxisomal ABC transporter COMATOSE allow differentiation between multiple functions in planta: insights from an allelic series. Molecular Biology of the Cell 20, 530-543, doi:10.1091/mbc.E08-070745 (2009).

10 Moriwaki, T. et al. Hormonal regulation of lateral root development in Arabidopsis modulated by MIZ1 and requirement of GNOM activity for MIZ1 function. Plant Physiol 157, 1209-1220, doi:10.1104/pp.111.186270 (2011).

11 Haseloff, J. in Methods in Cell Biology Vol. 58 (eds K.F. Sullivan \& S.A. Kay) Ch. 9, 139-151 (Academic Press, 1999).

12 Churchman, M. L. et al. SIAMESE, a plant-specific cell cycle regulator, controls endoreplication onset in Arabidopsis thaliana. The Plant Cell 18, 3145-3157, doi:10.1105/tpc.106.044834 (2006).

13 Karimi, M., Bleys, A., Vanderhaeghen, R. \& Hilson, P. Building blocks for plant gene assembly. Plant Physiol 145, 1183-1191, doi:10.1104/pp.107.110411 (2007).

14 Szymanski, D. B., Jilk, R. A., Pollock, S. M. \& Marks, M. D. Control of GL2 expression in Arabidopsis leaves and trichomes. Development 125, 1161-1171 (1998).

15 Galbraith, D. W., Harkins, K. R. \& Knapp, S. Systemic endopolyploidy in Arabidopsis thaliana. Plant Physiol 96, 985-989 (1991).

16 Zhang, C., Gong, F. C., Lambert, G. M. \& Galbraith, D. W. Cell type-specific characterization of nuclear DNA contents within complex tissues and organs. Plant Methods 1, 7, doi:10.1186/1746-4811-1-7 (2005).

17 Walter, A. et al. Spatio-temporal dynamics of expansion growth in roots: automatic quantification of diurnal course and temperature response by digital image sequence processing. Journal of Experimental Botany 53, 689-698 (2002).

18 Chavarria-Krauser, A. et al. Spatio-temporal quantification of differential growth processes in root growth zones based on a novel combination of image sequence 
processing and refined concepts describing curvature production. New Phytologist 177, 811-821, doi:10.1111/j.1469-8137.2007.02299.x (2008).

19 van der Weele, C. M. et al. A new algorithm for computational image analysis of deformable motion at high spatial and temporal resolution applied to root growth. Roughly uniform elongation in the meristem and also, after an abrupt acceleration, in the elongation zone. Plant Physiol 132, 1138-1148 (2003).

20 Shih, H. W., Miller, N. D., Dai, C., Spalding, E. P. \& Monshausen, G. B. The receptorlike kinase FERONIA is required for mechanical signal transduction in Arabidopsis seedlings. Current Biology : CB 24, 1887-1892, doi:10.1016/j.cub.2014.06.064 (2014).

21 Miller, N. D., Parks, B. M. \& Spalding, E. P. Computer-vision analysis of seedling responses to light and gravity. The Plant Journal 52, 374-381, doi:10.1111/j.1365313X.2007.03237.x (2007).

22 Basu, P., Pal, A., Lynch, J. P. \& Brown, K. M. A novel image-analysis technique for kinematic study of growth and curvature. Plant Physiol 145, 305-316, doi:10.1104/pp.107.103226 (2007).

23 Wells, D. M. et al. Recovering the dynamics of root growth and development using novel image acquisition and analysis methods. Philosophical Transactions of the Royal Society of London. Series B, Biological Sciences 367, 1517-1524, doi:10.1098/rstb.2011.0291 (2012).

24 Weinzaepfel, P., Revaud, J., Harchaoui, Z. \& Schmid, C. DeepFlow: Large displacement optical flow with deep matching. leee I Conf Comp Vis, 1385-1392, doi:10.1109/lccv.2013.175 (2013).

25 Shi, Y. G. \& Karl, W. C. Level set methods for dynamic tomography. 2004 2nd leee International Symposium on Biomedical Imaging: Macro to Nano, Vols 1 and 2, 620623 (2004).

26 Şuta, L., Bessy, F., Veja, C. \& Vaida, M.-F. in Intelligent Computer Communication and Processing (ICCP), 2012 IEEE International Conference on. 181-187 (IEEE).

27 Vandenboomgaard, R. \& Vanbalen, R. Methods for Fast Morphological Image Transforms Using Bitmapped Binary Images. Cvgip-Graph Model Im 54, 252-258, doi:Doi 10.1016/1049-9652(92)90055-3 (1992).

28 Dierckx, P. Curve and surface fitting with splines. (Clarendon, 1993). 
Table S1 Oligonucleotides used in this study, sequence in 5' to 3' direction

\begin{tabular}{|c|c|c|}
\hline \multicolumn{3}{|c|}{ Oligonucleotides for snrk2.2 snrk2.3 complementation } \\
\hline & forward oligonucleotide & reverse oligonucleotide \\
\hline \multicolumn{3}{|c|}{ SnRK2.2-GFP fusion } \\
\hline SnRK2.2 & ACATATTTGAGTGCAGTACAACGC & GAGAGCATAAACTATCTCTCCACT \\
\hline \multicolumn{3}{|c|}{ tissue-specific expression } \\
\hline $\begin{array}{l}\mathrm{RCH} 1 \\
\text { promoter }\end{array}$ & GGTACCCAGAACATACATCATCAAAGAATATCAT & CTCGAGAAGAGTTTTTTTCTTTGCATTTGGCTC \\
\hline $\begin{array}{l}\text { SnRK2.2 } \\
\text { promoter }\end{array}$ & GGTACCCAGTACAACGCAATCCACGATTTTG & CTCGAGTTTTTCTGATTCTTCTCTTCCTCTAT \\
\hline $\begin{array}{l}\text { SMB } \\
\text { promoter }\end{array}$ & $\begin{array}{l}\text { GGTACCTCGTTGAAGATGCCTGGATTTAATACT } \\
\text { G }\end{array}$ & CTCGAGTATCCTTACTCTTCTTTAAGCAAACTTT \\
\hline $\begin{array}{l}\text { WER } \\
\text { promoter }\end{array}$ & $\begin{array}{l}\text { GGTACCTTCCGACCTTAAAGCTCCTACAAAAAC } \\
\text { ATGAAGAT }\end{array}$ & $\begin{array}{l}\text { CTCGAGTCTTTTTGTTTCTTTGAATGATAGACGA } \\
\text { GAGAGAT }\end{array}$ \\
\hline SnRK2.2 & CTCGAGATGGATCCGGCGACTAATTCACCGAT & $\begin{array}{l}\text { CTGCAGTCAGAGAGCATAAACTATCTCTTCCAC } \\
\text { TAC }\end{array}$ \\
\hline \multicolumn{3}{|c|}{ Oligonucleotides for SIM overexpression } \\
\hline En7 & GCTCCATTAGTCCATATACACAGTTGAC & TTAAGATTCTGAGATTCACGAAGAAAAGCAGC \\
\hline Co2 & TAACTCCATTATTTACGACTGTGCCACTCT & AAACTCTTGTTGCATTATTGTCAAATCCTT \\
\hline GL2 & GACCTAAGTTTCCTTCACTATACG & ACAAATCCTGTCCCTAGCTAGC \\
\hline \multicolumn{3}{|c|}{ Oligonucleotides for miz1 complementation } \\
\hline \multicolumn{3}{|c|}{ tissue-specific MIZ1-GFP expression with HSP terminator } \\
\hline $\begin{array}{l}\text { WER } \\
\text { promoter }\end{array}$ & $\begin{array}{l}\text { CCAAGCTTGCATGCCTGCAGGGTCTTTCTTTAT } \\
\text { TCTTCTC }\end{array}$ & $\begin{array}{l}\text { TTGGTATGGCACCATTCTTTTTGTTTCTTTGAAT } \\
\text { G }\end{array}$ \\
\hline $\begin{array}{l}\text { MIZ1 for } \\
\text { WER } \\
\text { promoter }\end{array}$ & $\begin{array}{l}\text { AAAGAAACAAAAAGAATGGTGCCATACCAAGAA } \\
\text { CT }\end{array}$ & $\begin{array}{l}\text { CTTCATCTTCATAAGAGCTCTCAAATTCTCTTTA } \\
\text { AAAACC }\end{array}$ \\
\hline $\begin{array}{l}\text { PIN2 } \\
\text { promoter }\end{array}$ & $\begin{array}{l}\text { CCAAGCTTGCATGCCTGCAGCATCCTGTTTTAT } \\
\text { CAGGCTA }\end{array}$ & $\begin{array}{l}\text { TTGGTATGGCACCATTTTGATTTACTTTTTCCGG } \\
\mathrm{C}\end{array}$ \\
\hline $\begin{array}{l}\text { MIZ1 for } \\
\text { PIN2 } \\
\text { promoter }\end{array}$ & $\begin{array}{l}\text { AAAAAGTAAATCAAAATGGTGCCATACCAAGAA } \\
\text { CT }\end{array}$ & $\begin{array}{l}\text { CTTCATCTTCATAAGAGCTCTCAAATTCTCTTTA } \\
\text { AAAACC }\end{array}$ \\
\hline \multicolumn{3}{|c|}{ tissue-specific MIZ1-GFP expression with MIZ1 terminator } \\
\hline $\begin{array}{l}\mathrm{RCH} 1 \\
\text { promoter }\end{array}$ & $\begin{array}{l}\text { CAGTGGTACCCAGAACATACATCATCAAAGAAT } \\
\text { ATC }\end{array}$ & $\begin{array}{l}\text { GCTACGGCCGAAGAGTTTTTTTCTTTGCATTTG } \\
\text { GC }\end{array}$ \\
\hline $\begin{array}{l}\text { SMB } \\
\text { promoter }\end{array}$ & $\begin{array}{l}\text { CAGTGGTACCTCGTTGAAGATGCCTGGATTTAA } \\
\text { TAC }\end{array}$ & $\begin{array}{l}\text { GCTACGGCCGTATCCTTACTCTTCTTTAAGCAA } \\
\text { AC }\end{array}$ \\
\hline $\begin{array}{l}\text { SCR } \\
\text { promoter }\end{array}$ & $\begin{array}{l}\text { CAGTGGTACCAATTTTGAATCCATTCTCAAAGCT } \\
\text { TTG }\end{array}$ & $\begin{array}{l}\text { GCTACGGCCGGGAGATTGAAGGGTTGTTGGTC } \\
\text { GTGAG }\end{array}$ \\
\hline $\begin{array}{l}\text { Co2 } \\
\text { promoter }\end{array}$ & $\begin{array}{l}\text { CAGTGGTACCTAACTCCATTATTTACGACTGTG } \\
\text { CC }\end{array}$ & $\begin{array}{l}\text { GCTACGGCCGAAACTCTTGTTGCATTATTGTCA } \\
\text { AATC }\end{array}$ \\
\hline $\begin{array}{l}\text { WER } \\
\text { promoter }\end{array}$ & $\begin{array}{l}\text { CAGTGGTACCTTCCGACCTTAAAGCTCCTACAA } \\
\text { AAAC }\end{array}$ & $\begin{array}{l}\text { GCTACGGCCGTCTTTTTGTTTCTTTGAATGATA } \\
\text { GAC }\end{array}$ \\
\hline $\begin{array}{l}\text { COR } \\
\text { promoter }\end{array}$ & $\begin{array}{l}\text { CAGTGGTACCCGTGCGGTCAAATAACAGAAGAA } \\
\text { ATG }\end{array}$ & $\begin{array}{l}\text { GCTACGGCCGGGTTTTGGCTAATGTGATTGTGT } \\
\text { AG }\end{array}$ \\
\hline \multicolumn{3}{|c|}{ Oligonucleotides for qRT-PCR } \\
\hline SnRK2.2 & GGAGCTTATCCATTCGAGGA & CCTCTGGGATCGAGTATGTGA \\
\hline UBA & AGTGGAGAGGCTGCAGAAGA & CTCGGGTAGCACGAGCTTTA \\
\hline
\end{tabular}




\title{
Root hydrotropism is controlled via a cortex-specific growth mechanism - Supplementary Note 1
}

\author{
Daniela Dietrich, Lei Pang, Akie Kobayashi, John A. Fozard, \\ Véronique Boudolf, Rahul Bhosale, Regina Antoni, Tuan Nguyen, \\ Sotaro Hiratsuka, Nobuharu Fujii, Yutaka Miyazawa, Tae-Woong Bae, \\ Darren M. Wells, Markus R. Owen, Leah R. Band, Rosemary J. Dyson, \\ Oliver E. Jensen, John R. King, Saoirse R. Tracy, Craig J. Sturrock, \\ Sacha J. Mooney, Jeremy A. Roberts, Rishikesh P. Bhalerao, \\ José R. Dinneny, Pedro L. Rodriguez, Akira Nagatani, \\ Yoichiroh Hosokawa, Tobias I. Baskin, Tony P. Pridmore, \\ Lieven De Veylder, Hideyuki Takahashi and Malcolm J. Bennett
}

\section{Estimating water potential gradients and ABA re- sponses during hydrotropism}

\subsection{Model preliminaries}

We wish to understand how hydrotropic responses are effected. The approach we adopt is a straightforward one, given that the mechanisms by which water potential is sensed, and how differences in growth rate are generated, are not fully understood.

We have observed that treatment of roots growing on plates without sorbitol with $100 \mathrm{nM}$ ABA changed their growth rate from $6.23 \mathrm{~mm} /$ day to 8.28 $\mathrm{mm} /$ day (Figure 3). In the experiments of van der Weele [9], it was found (see Figure 3 of [9]) that plants growing on plates with small amounts of PEG (which is similar in effect to low levels of sorbitol) actually grew faster than on normal culture plates (Hoagland solution); roots in the presence of PEG (corresponding to an external water potential of $-0.23 \mathrm{MPa}$ ) grew at about $6 \mathrm{~mm} /$ day, compared with roots on Hoagland solution (external water potential of $-0.1 \mathrm{MPa}$ ), which grew at about $5 \mathrm{~mm} /$ day.

We are interested in how large an ABA gradient is needed to generate observed levels of root bending. From the measurements of root-tip angle (Figure $2 \mathrm{~b}$ ), we estimate that hydrotropic bending occurs at a rate of about 20 degrees in 6 hours, or slightly faster than 3 degrees per hour. We can make a 
crude estimate of the difference in ABA concentration between cortical cells on the wet and dry sides of the root that is required to generate this observed rate of bending. We also wish to relate this to an estimate of the relative difference in water potential between the wet and dry sides of the root.

\subsection{Estimate of water potential gradient}

In the split agar-based hydrotropism assays, a cut is made in the agar plate and sorbitol-containing agar placed on one side of this cut. Using the diffusion equation, the sorbitol concentration (and consequently the water potential) can be calculated. From the solution of the diffusion equation, the sorbitol concentration $c$ is found to be

$$
c=\operatorname{Cerfc}\left(\frac{x}{2 \sqrt{D t}}\right),
$$

where $x$ is distance from the cut, $C=400 \mathrm{mM}$ is the initial concentration of sorbitol in the sorbitol-containing agar, the diffusion coefficient $D$ is estimated as $D=10^{-5} \mathrm{~cm}^{2} / \mathrm{hr}$ from [5], and erfc denotes the complementary error function. The water potential $\Psi$ is related to the sorbitol concentration by the van ' $t$ Hoff equation $\Psi=-c i R T$, where $i$ is the van 't Hoff factor ( $i \simeq 1$ for sorbitol, as it is not an electrolyte), $R=8.314 \mathrm{kPa} \mathrm{M}^{-1} \mathrm{~K}^{-1}$ is the gas constant and $T=298 \mathrm{~K}$ is room temperature; from this we find that $400 \mathrm{mM}$ sorbitol corresponds to a water potential of about $-1 \mathrm{MPa}$. Thus

$$
\Psi=-A \operatorname{erfc}\left(\frac{x}{2 \sqrt{D t}}\right),
$$

where $A \simeq 1 \mathrm{MPa}$. These estimates give a spatial distribution of water potential that is in good agreement with the experimental measurements of [8]. The root tip is placed approximately at $2.5 \mathrm{~mm}$ from the cut in the plate at the start of the experiment. From evaluation of this water potential distribution (Figure S1) both sides of the root can be seen to be at similar external water potential, with the maximum absolute difference in water potential between the two sides of the root $(<10 \mathrm{kPa}$, Figure S1(a)) being less than 3\% of the maximum (absolute) water potential experienced at the root midline $(\simeq-400 \mathrm{kPa}$, Figure S1(b)).

\subsection{Estimate of ABA gradient}

In this calculation, we assume that the cortical cells are free to grow unimpeded by the neighbouring cells, both for straight growing roots treated with ABA and for roots undergoing hydrotropism (i.e. the other tissues exert no axial stresses, but simply keep the cortical cells separated by a constant distance). The bending rate of 3 degrees per hour is equivalent to 0.05 radians per hour.

From measurements of confocal images of Arabidopsis thaliana roots, the cortical cells on the wet and dry side of the root are separated by approximately $60 \mu \mathrm{m}$. Using this distance measurement, the estimate of the bending rate, and the formula for the circumference of a circular arc (Figure S2), we have that the 
cortical cells on the dry side must be growing faster than the cortical cells on the wet side by $60 * 0.05=3 \mu \mathrm{m} / \mathrm{hr}=72 \mu \mathrm{m} /$ day.

From the ABA application experiments, 100nM ABA application induced an increase in root growth of $2.05 \mathrm{~mm} /$ day. Linearly interpolating, we find that the difference in ABA concentration between the two sides should be of the order of $4 \mathrm{nM}$. This is a relatively small difference in ABA concentration, compatible with the idea that lateral ABA gradients are unlikely to be detectable using currently available experimental techniques. Taking $100 \mathrm{nM}$ as a physiologically relevant $\mathrm{ABA}$ concentration, this $4 \%$ difference between the two sides is in fact of the same order of magnitude as the relative difference in water potential between the two sides of the root.

\section{Midline bending models}

\subsection{Model description}

In the split-agar based hydrotropism assay, roots were grown on the surface of an agar gel and remained in contact with it throughout the experiment, so the geometry of a particular root can be represented by a two dimensional curve through its midline. A complete description of such a curve is supplied by the midline curvature, $\kappa$, and its stretch (logarithmic strain), $\epsilon_{m}$. (We use the subscript $m$ to indicate that this is the stretch of the midline; we will later consider the stretch in walls that are offset from the midline.) The evolution of these quantities (the rate of change of curvature, $\dot{\kappa}$, and the axial elongation rate, $\dot{\epsilon}_{m}$ ) can be related to the material properties of individual cell walls and to the cellular turgor pressures using a mechanical model based (in a sense indicated below) on that described by [3].

A representative transverse cross-section (perpendicular to the root midline) of an Arabidopsis root was used (based on Figure IA of [1]). This was converted into a geometric template (Figure 4c), in which cells occupy polygonal regions, and the edges bounding each polygon represent cell walls. These cell walls can be thought of as one-dimensional lines lying in the two-dimensional plane of the cross-section; they will each have an associated thickness. The cross-section of the root was assumed to be uniform in the region of interest, and thicknesses $h$ were assigned to each cell wall following the measurements of [3]; these are listed in Table S1(a).

Cell walls were treated as viscoplastic along the axis of the root, with kinematic viscosity, $\mu$, and yield stress, $y$. As the diameter of the root is approximately constant shootwards of the lateral root cap, walls are considered to be inextensible in the transverse direction. This results in the geometry of the transverse cross-section remaining constant as the root elongates, and it was further assumed that the thickness of each wall segment, $h$, remains constant through the production of new wall material. The turgor pressure $P$ was taken to be constant and the same within all cells.

Integrating over the wall thickness, an axial extensibility $\phi$ and yield $Y$ was 
associated with each wall section, namely

$$
\phi=\frac{1}{4 \mu h}, \quad Y=y h,
$$

the factor of four in the axial extensibility being a consequence of the flow within an elongating viscous sheet [4]. Once we have integrated over the wallthickness, we treat each wall as a one-dimensional line segment lying in the plane of the cross-section. Changes in the curvature of the midline are associated with walls at different distances from the midline having different axial elongation rates. A line segment parallel to the root axis, at fixed distance $\eta$ from the midline of the root ( $\eta>0$ being the dry side of the root), can be found from geometrical considerations (see Section 2.5) to have elongation rate

$$
\dot{\epsilon}(s, \eta, t)=\dot{\epsilon}_{m}-\dot{\kappa} \eta
$$

where $\dot{\epsilon}_{m}$ is the elongation rate of the root midline (an approximation derived by taking the curvature to be small compared to the reciprocal of the radius of the root cross-section). In growing parts of the root, each wall section then exerts an axial tension force

$$
T=Y+\phi^{-1} \dot{\epsilon}
$$

per unit length in the transverse cross section, cf. [6]. It was assumed that the walls in each transverse cross section are either all in yield $(T>Y)$, where the root is growing $\left(\dot{\epsilon}_{m}>0\right)$, or undergoing no stretching or bending in regions where the root is not growing $\left(\dot{\epsilon}_{m}=\dot{\kappa}=0\right)$.

From (5), each wall exerts an axial tension force

$$
T=Y+\phi^{-1}\left(\dot{\epsilon}_{m}-\dot{\kappa} \eta\right)
$$

per unit length in the cross-section, whilst the turgor pressure exerts an axial force $P$ per unit area over the portion of the cross-section occupied by the cells. Provided there are no external forces acting on the root, the total force (parallel to the root midline) and moment (about the intersection of the midline and the cross section) must be zero over each transverse cross section; these conditions become

$$
\begin{aligned}
\int_{w}\left(\phi^{-1} \dot{\epsilon}_{m}+Y\right) \mathrm{d} x-\dot{\kappa} \int_{w} \phi^{-1} \eta \mathrm{d} x & =P \mathcal{A}, \\
\dot{\kappa} \int_{w} \phi^{-1} \eta^{2} \mathrm{~d} x & =\int_{w}\left(Y+\phi^{-1} \dot{\epsilon}_{m}\right) \eta \mathrm{d} x,
\end{aligned}
$$

where the subscript $w$ denotes a line integral over all walls in the cross section, and $x$ measures distance along each wall. If the stretching from curvature of the midline is small compared to the stretching from elongation of the midline, i.e $\dot{\kappa} H$ is small compared to $\dot{\epsilon}_{m}$, where $H$ is the radius of the root, the second term 
of the left hand side of (7) may be ignored, giving elongation and curvature generation rates

$$
\dot{\epsilon}_{m}=\frac{P \mathcal{A}-\int_{w} Y \mathrm{~d} x}{\int_{w} \phi^{-1} \mathrm{~d} x}, \quad \dot{\kappa}=\frac{\int_{w} Y \eta \mathrm{d} x+\dot{\epsilon}_{m} \int_{w} \phi^{-1} \eta \mathrm{d} x}{\int_{w} \phi^{-1} \eta^{2} \mathrm{~d} x} .
$$

with $\mathcal{A}$ being the area occupied by the cells in the cross section. These equations assume that the cross section is (approximately) symmetric about $\eta=0$, such that

$$
\int \eta \mathrm{d} A \approx 0
$$

where this area integral is over the region in the cross-section occupied by the cells.

\subsection{Mechanical properties of roots growing without hydrotropic gradient}

A simplified, but representative, growth profile in the absence of a hydrotropic stimulus takes the form

$$
\dot{\epsilon}_{m}(s)= \begin{cases}\dot{\epsilon}_{1} & 0 \leq s<s_{1} \\ \dot{\epsilon}_{2} & s_{1} \leq s<s_{2} \\ 0 & s_{2} \leq s\end{cases}
$$

as illustrated in Figure S3. Such a growth profile is based upon those measured by [7] and [2], although the transitions between different growth sections are made abrupt, which simplifies the model. Here $s$ is distance along the root midline, measured from the tip of the root, $0 \leq s<s_{1}$ is the extent of the slowly growing zone in the root, encompassing the meristem and "transition zones", $s_{1} \leq s \leq s_{2}$ corresponds to the "elongation zone" within which cells undergo rapid expansion, and $s_{2} \leq s$ is the "maturation zone" (in which root elongation has stopped).

Little is known about the variation in cell-wall material properties throughout the root: in the model here, changes in growth rate can be caused by changes in yield stress, extensibility, or in both quantities simultaneously. In earlier work on gravitropic bending [3], it was found that bending in regions of slow elongation is more likely to be generated by changes in wall yield stress. This can be seen in (9), where the term depending on wall extensibility has a prefactor of $\dot{\epsilon}_{m}$, unlike the term depending on wall yield. A plausible approach, adopted here, is to take both the wall viscosity and yield stress to be uniform within each cross section, so that $\mu \equiv \mu(s), y \equiv y(s)$. The yield stress was taken constant within the meristem $\left(0 \leq s \leq s_{1}\right)$, zero within the meristem $\left(s_{1}<s \leq s_{2}\right)$ and large elsewhere, whilst the extensibility was taken constant 
within the growing portion of the $\operatorname{root}\left(0 \leq s \leq s_{2}\right)$; explicitly

$$
y(s)=\left\{\begin{array}{ll}
y_{0} & 0 \leq s \leq s_{1} \\
0 & s_{1}<s \leq s_{2}, \\
\infty & s_{2}<s
\end{array} \quad \mu(s)= \begin{cases}\mu_{0} & 0 \leq s \leq s_{2} \\
\infty & s_{2}<s\end{cases}\right.
$$

The parameters $\mu_{0}$ and $y_{0}$ were chosen such that the root adopts the growth profile (11), specifically

$$
\mu_{0}=\frac{P \mathcal{A}}{4 \dot{\epsilon}_{2} A_{w}}, \quad y_{0}=\frac{P \mathcal{A}\left(1-\frac{\dot{\epsilon}_{1}}{\dot{\epsilon}_{2}}\right)}{A_{w}},
$$

where the total area of walls in each transverse cross-section, $A_{w}$, was calculated using

$$
A_{w}=\int_{w} h \mathrm{~d} x .
$$

Although (in the absence of a hydrotropic gradient) the material parameters $\mu$ and $y$ are uniform in each transverse cross-section, the resulting yields and extensibilities $Y$ and $\phi$ are not the same for all wall sections, but depend on the thickness $h$ of each individual wall through (3).

\subsection{Modelling hydrotropic bending}

It was assumed that, following a hydrotropic stimulus, cortical cells on the dry side of the root enter rapid elongation closer to the root apex than during normal growth, at $s=s_{1}-\delta$ rather than at $s=s_{1}$, where $\delta>0$ is chosen to be about three cortical cell-lengths (see Figure 4c, where the cortical cells undergoing early onset of elongation are indicated in pink). This corresponds to a change in the yield stresses of the cortical cell walls on the dry side, in the region $s_{1}-\delta<s<s_{1}$, from $y=y_{0}$ to $y=0$; the yield stress of the walls of the other cells in this region remains unchanged. It was asumed that this early entry into rapid elongation occurs for the period of time $0 \leq t \leq T$, subsequent to the roots being placed in the presence of a hydrotropic gradient. The parameters here are listed in Table S1(b).

By (9), the elongation rate of the root midline was given, for $0 \leq t \leq T$, by

$$
\dot{\epsilon}(s)= \begin{cases}\dot{\epsilon}_{1} & 0 \leq s<s_{1}-\delta \\ \dot{\epsilon}_{1}+\left(\dot{\epsilon}_{2}-\dot{\epsilon}_{1}\right) \frac{1}{A_{w}} \int_{c w} h \mathrm{~d} x & s_{1}-\delta \leq s<s_{1} \\ \dot{\epsilon}_{2} & s_{1} \leq s<s_{2} \\ 0 & s_{2} \leq s\end{cases}
$$

where the subscript $c w$ denotes integrals over the cortical cell walls on the dry side of the $\operatorname{root} \eta>0$. 
In the region $s_{1}-\delta \leq s<s_{1}$, we have that $y=y_{0}$ for all cell walls except those of the cortical cells on the dry side of the root. Using (3), (9) can be written as

$$
\dot{\kappa}=\frac{\int_{w} y h \eta \mathrm{d} x+4 \mu_{0} \dot{\epsilon}_{m} \int_{w} h \eta \mathrm{d} x}{4 \mu_{0} \int_{w} \eta^{2} \mathrm{~d} x},
$$

and the symmetry assumption (20) allows this to be simplified to

$$
\dot{\kappa}=-\frac{y_{0}}{4 \mu_{0}} \frac{\int_{c w} h \eta \mathrm{d} x}{\int_{w} \eta^{2} \mathrm{~d} x} .
$$

From (13),

$$
\frac{y_{0}}{4 \mu_{0}}=\left(\dot{\epsilon}_{2}-\dot{\epsilon}_{1}\right)
$$

from which its found that curvature of the root midline generated at a rate

$$
\dot{\mathcal{K}}(s)= \begin{cases}0 & 0 \leq s<s_{1}-\delta \\ -\left(\dot{\epsilon}_{2}-\dot{\epsilon}_{1}\right) \frac{\int_{c w} h \eta \mathrm{d} x}{\int_{w} h \eta^{2} \mathrm{~d} x} & s_{1}-\delta \leq s<s_{1} \\ 0 & s_{1} \leq s\end{cases}
$$

the curvature in $s_{1}-\delta \leq s<s_{1}$ having negative sign as the cortical cell walls on the dry side lie in $\eta>0$. This calculation further assumed that the root template was approximately symmetrical, such that

$$
\int_{w} h \eta \mathrm{d} x \simeq 0 .
$$

Using the template shown in Figure 4c, and the cell-wall thicknesses listed in Table S1(a), we estimate the integrals appearing in (15) and (19) to be

$$
\begin{aligned}
\frac{1}{A_{w}} \int_{c w} h \mathrm{~d} x & \simeq 0.11, & \frac{1}{A_{w}} \int_{c w} h \eta \mathrm{d} x & \simeq 2.8 \mu \mathrm{m} \\
\frac{1}{A_{w}} \int_{w} h \eta^{2} \mathrm{~d} x & \simeq 1200 \mu \mathrm{m}^{2}, & \frac{1}{A_{w}} \int_{c w} h \eta^{2} \mathrm{~d} x & \simeq 86 \mu \mathrm{m}^{2} .
\end{aligned}
$$

Using the parameter values in Table S1(b), we estimate the elongation and curvature rates within the zone of bending $s_{1}-\delta \leq s<s_{1}$ to be

$$
\dot{\epsilon}_{m} \simeq 0.13 \mathrm{hr}^{-1} \quad \dot{\kappa} \simeq-0.0007 \text { radians } \mu \mathrm{m}^{-1} \mathrm{hr}^{-1} .
$$

\subsection{Numerical calculation of bending profiles}

Whilst in some circumstances it is practical to calculate the bending pattern analytically [3], here the problem was treated numerically. The midline was initially taken to be straight, with length $L_{0} \geq s_{2}$, and was split into $N$ sections, 
with lengths $l_{n}=L_{0} / N$ and curvatures $\kappa_{n}=0$, for $n=1, \ldots, N$. Parameter values are listed in Table S1(c). These discrete variables evolve according to

$$
\frac{d \log \left(l_{n}\right)}{d t}=\frac{1}{l_{n}} \int_{S_{n-1}}^{S_{n}} \dot{\epsilon}(\hat{s}, t) \mathrm{d} \hat{s}, \quad \frac{d \kappa_{n}}{d t}=\frac{1}{l_{n}} \int_{S_{n-1}}^{S_{n}} \dot{\kappa}(\hat{s}, t) \mathrm{d} \hat{s},
$$

where

$$
S_{0}=0, \quad S_{n}=\sum_{m=1}^{n} l_{m}
$$

for $n=1, \ldots, N$. Integration was performed using a simple forward Euler scheme (with variables $\log \left(l_{n}\right)$ and $\kappa_{n}$ ), approximating the integrals using the values of their integrands at the midpoints of each section,

$$
\begin{aligned}
& l_{n}(t+\Delta t)=l_{n}(t) \exp \left(\dot{\epsilon}\left(\frac{S_{n-1}+S_{n}}{2}, t\right) \Delta t\right), \\
& \kappa_{n}(t+\Delta t)=\kappa_{n}(t)+\Delta t \dot{\kappa}\left(\frac{S_{n-1}+S_{n}}{2}, t\right),
\end{aligned}
$$

where $\Delta t$ is the fixed timestep. Sections were split into two, each with half the length of the parent and the same curvature, when they exceeded a threshold length $l_{C}$, in order to maintain the spatial resolution of the simulation. The angle of the root-tip (relative to that at the basal end) was given by $\theta=$ $-\sum_{m=1}^{N} l_{m} \kappa_{m}$, the negative sign being taken as the direction of the root tip is opposite to the direction of increasing $s$. Simulation results of tip angle against time are shown in Figure 2i.

\subsection{Elongation rate of curves parallel to bending root midline}

Here we give details of the derivation of the relationship (4) between the elongation rate $\dot{\epsilon}$ of a wall parallel to the root midline, and the bending and stretching rates $\left(\dot{\kappa}, \dot{\epsilon}_{m}\right)$ of the root midline.

Consider a short curve of length $l$, at constant distance $\eta$ from the midline of the root, measured in the direction of the normal to the midline $n$ (see Figure S4). Also assume for now that $\kappa>0$. Then this curve has radius of curvature $\kappa^{-1}-\eta$, and subtends an angle $\kappa l /(1-\kappa \eta)$ at the centre of curvature. It therefore projects onto a curve of length $l_{0}$ along the root midline, where $l$ and $l_{0}$ are related through

$$
l=(1-\kappa \eta) l_{0},
$$

The same expression can be seen to hold for $\kappa \leq 0$. Differentiating (28) with 
respect to time, the axial elongation rate of this curve is therefore given by

$$
\begin{aligned}
\dot{\epsilon}(s, \eta, t) \equiv \frac{1}{l} \frac{d l}{d t} & =\frac{1}{l_{0}(1-\kappa \eta)}\left((1-\kappa \eta) \frac{\mathrm{d} l_{0}}{\mathrm{~d} t}-\dot{\kappa} l_{0} \eta\right) \\
& =\frac{1}{l_{0}} \frac{\mathrm{d} l_{0}}{\mathrm{~d} t}-\frac{\dot{\kappa} \eta}{1-\kappa \eta} \\
& =\dot{\epsilon}_{m}-\frac{\dot{\kappa} \eta}{1-\kappa \eta} .
\end{aligned}
$$

This can be approximated, again taking the limit $\kappa H \ll 1$, by

$$
\dot{\epsilon}(s, \eta, t)=\dot{\epsilon}_{m}-\dot{\kappa} \eta .
$$

\section{References}

[1] I. Casimiro, A. Marchant, R. P. Bhalerao, T. Beeckman, S. Dhooge, R. Swarup, N. Graham, D. Inze, G. Sandberg, P. J. Casero, and M. Bennett. Auxin transport promotes Arabidopsis lateral root initiation. Plant Cell, 13:843-852, 2001.

[2] A. Chavarria-Krauser, K. A. Nagel, K. Palme, U. Schurr, A. Walter, and H. Scharr. Spatio-temporal quantification of differential growth processes in root growth zones based on a novel combination of image sequence processing and refined concepts describing curvature production. New Phytol., 177:843-852, 2008.

[3] R. J. Dyson, G. Vizcay-Barrena, L. R. Band, A. N. Fernandes, A. P. French, J. A. Fozard, T. C. Hodgman, K. Kenobi, T. P. Pridmore, M. Stout, D. M. Wells, M. H. Wilson, M. J. Bennett, and O. E. Jensen. Mechanical modelling quantifies the functional importance of outer tissue layers during root elongation and bending. New Phytol., 202:1212-1222, 2014.

[4] RJ Dyson and OE Jensen. A fibre-reinforced fluid model of anisotropic plant cell growth. J. Fluid Mech., 655:472-503, 2010.

[5] L. Lebrun and G. A. Junter. Diffusion of sucrose and dextran through agar gel membranes. Enzyme Microb. Technol., 15(12):1057-1062, Dec 1993.

[6] J. A. Lockhart. An analysis of irreversible plant cell elongation. J. Theor. Biol., 8:264-275, 1965.

[7] W. S. Peters and T. I. Baskin. Tailor-made composite functions as tools in model choice: the case of sigmoidal vs bi-linear growth profiles. Plant Methods, 2:11, 2006.

[8] N. Takahashi, N. Goto, K. Okada, and H. Takahashi. Hydrotropism in abscisic acid, wavy, and gravitropic mutants of Arabidopsis thaliana. Planta, 216:203-211, 2002. 
[9] C. M. van der Weele, W. G. Spollen, R. E. Sharp, and T. I. Baskin. Growth of Arabidopsis thaliana seedlings under water deficit studied by control of water potential in nutrient-agar media. J. Exp. Bot., 51:1555-1562, 2000. 
(a)

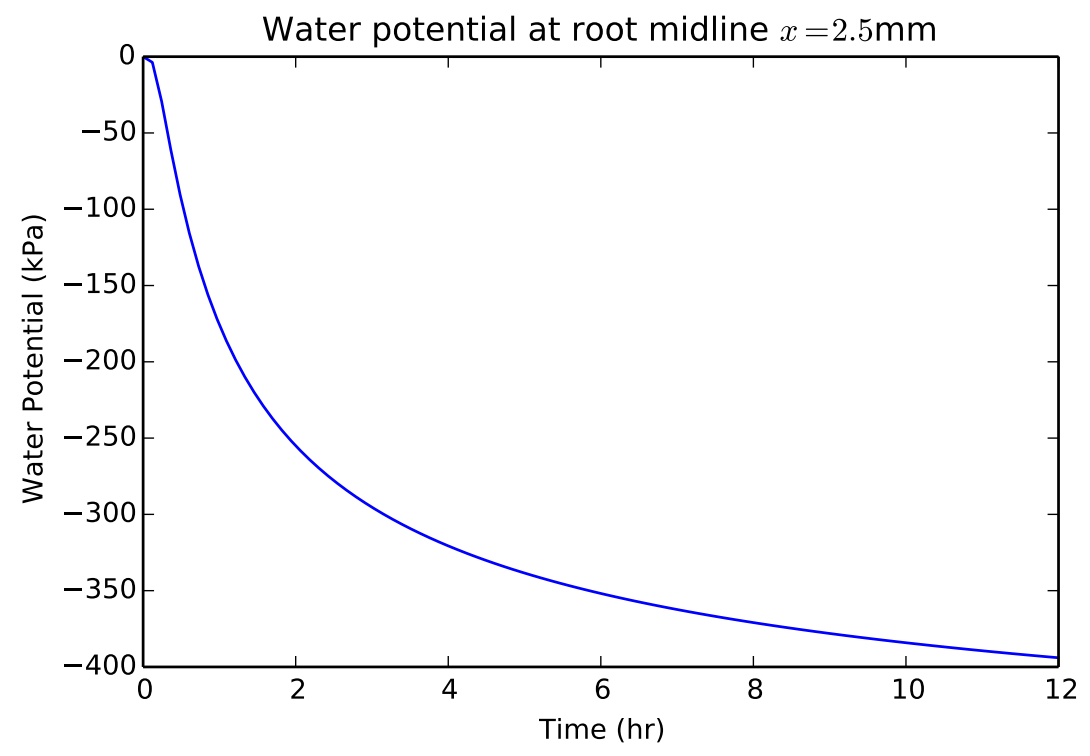

(b)

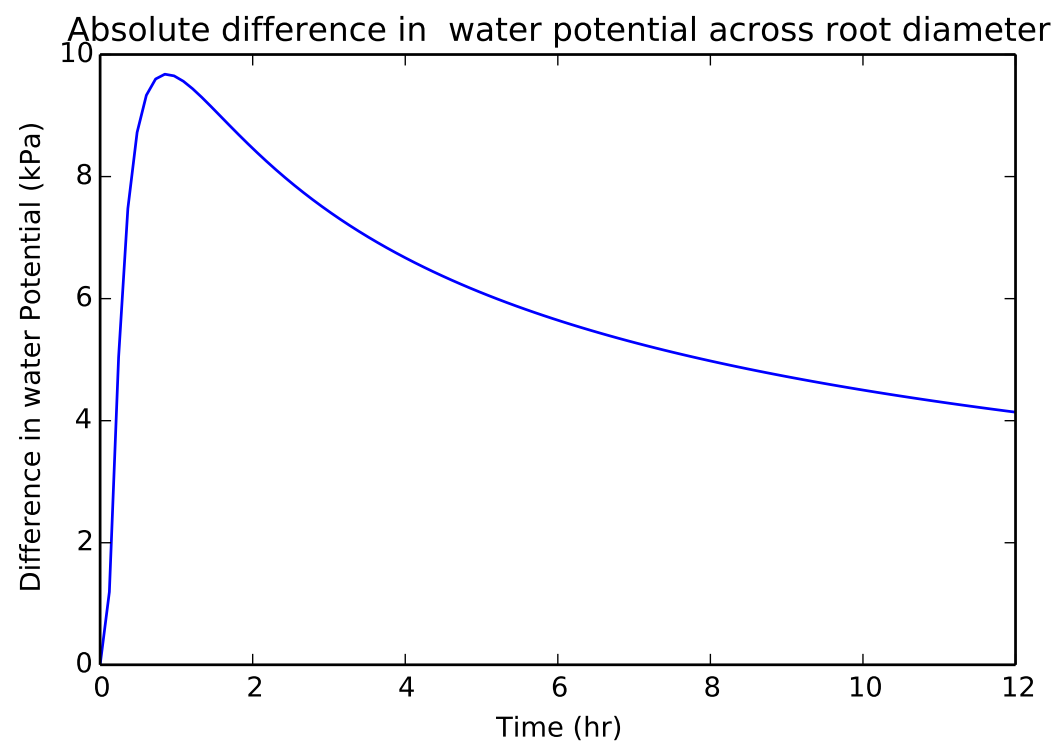

Figure S1: Water potential in the hydrotropism assay, calculated from equation (2). (a) Water potential at $2.5 \mathrm{~mm}$ from the cut in the agar plate. (b) Absolute water potential difference between two points at $2.5 \mathrm{~mm}-R$ and $2.5 \mathrm{~mm}+R$ from the cut, where $R=50 \mu \mathrm{m}$ is an estimate of root radius. 


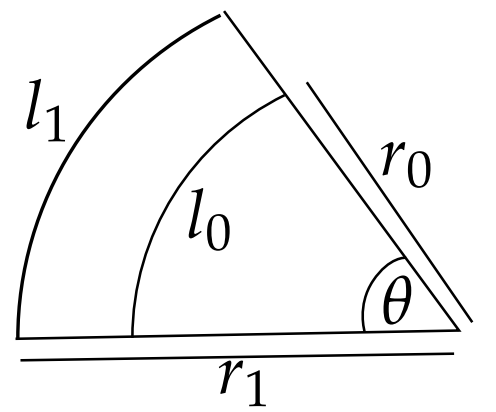

$$
\begin{aligned}
& l_{0}=r_{0} \theta \\
& l_{1}=r_{1} \theta \\
& \theta=\frac{l_{1}-l_{0}}{r_{1}-r_{0}}
\end{aligned}
$$

Figure S2: Relationship between bending angle, $\theta$, and the difference in the lengths of the cortical cells on each side $l_{1}-l_{0}$, where here $r_{1}-r_{0} \approx 60 \mu \mathrm{m}$ is the distance between the centres of the cortical cell files on the wet and dry side of the root. 

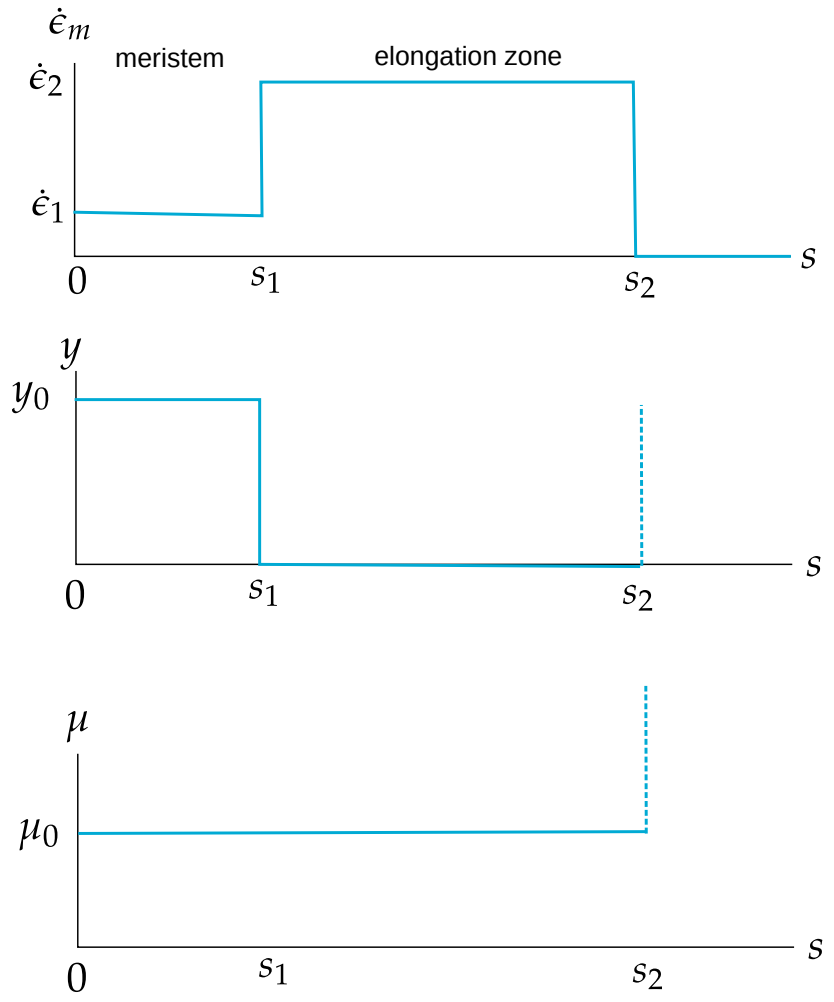

Figure S3: Growth profile $\left(\dot{\epsilon}_{m}\right)$, yield stress $(y)$ and viscosity $(\mu)$ assumed for straight growing roots in the absence of a hydrotropic stimulus 


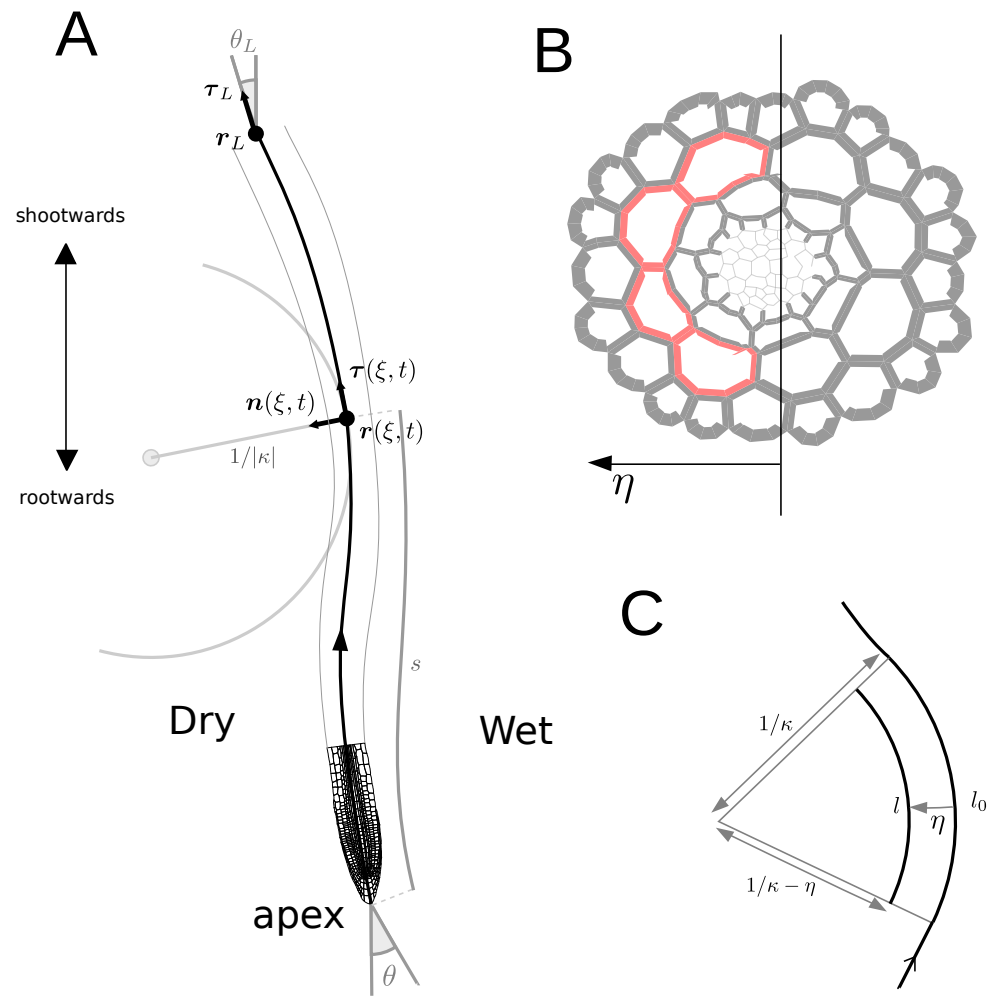

Figure S4: (A) Geometric quantities in midline model. (B) Cross section used for simulation. Line thicknesses correspond to the wall thicknesses used in the bending calculation. Cortical walls on the dry side of the root indicated in pink. (C) Projection of a line of length $l$, at a distance $\eta$ from the root midline, onto a length $l_{0}$ along the root midline (see equation (28)). 


\begin{tabular}{|l|l|l|}
\hline \multicolumn{2}{|c|}{ Cell Wall Type } & Thickness (nm) \\
\hline \multirow{2}{*}{ Pericycle } & $\mathrm{R}$ & 38 \\
& $\mathrm{O}$ & 47 \\
\hline \multirow{3}{*}{ Endodermis } & $\mathrm{R}$ & 45 \\
& $\mathrm{I}$ & 48 \\
& $\mathrm{O}$ & 70 \\
\hline \multirow{3}{*}{ Cortex } & $\mathrm{R}$ & 77 \\
& $\mathrm{I}$ & 80 \\
\hline \multirow{3}{*}{ Epidermis } & $\mathrm{O}$ & 105 \\
\hline & $\mathrm{R}$ & 86 \\
& $\mathrm{I}$ & 93 \\
& $\mathrm{O}$ & 198 \\
\hline
\end{tabular}

(b) \begin{tabular}{cc}
\hline$s_{1}$ & $400 \mu \mathrm{m}$ \\
$s_{2}$ & $1000 \mu \mathrm{m}$ \\
$\delta$ & $50 \mu \mathrm{m}$ \\
\hline$\dot{\epsilon}_{1}$ & $0.1 \mathrm{hr}^{-1}$ \\
$\dot{\epsilon}_{2}$ & $0.4 \mathrm{hr}^{-1}$ \\
\hline$T$ & $2 \mathrm{hr}$ \\
\hline
\end{tabular}

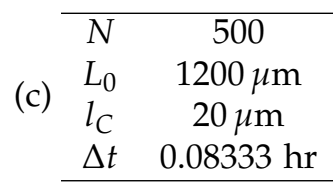

Table S1: (a) Cell wall thicknesses. R, I, O indicates radial, inner and outer cell walls, respectively. Measurements from [3]. (b) Parameter values for growth profile used in model. (c) Parameter values for numerical simulation of bending profile. 


\title{
Root hydrotropism is controlled via a cortex-specific growth mechanism - Supplementary Note 2
}

\author{
Daniela Dietrich, Lei Pang, Akie Kobayashi, \\ John A. Fozard, Véronique Boudolf, Rahul Bhosale, \\ Regina Antoni, Tuan Nguyen, Sotaro Hiratsuka, \\ Nobuharu Fujii, Yutaka Miyazawa, Tae-Woong Bae, \\ Darren M. Wells, Markus R. Owen, Leah R. Band, \\ Rosemary J. Dyson, Oliver E. Jensen, John R. King, \\ Saoirse R. Tracy, Craig J. Sturrock, Sacha J. Mooney, \\ Jeremy A. Roberts, Rishikesh P. Bhalerao, José R. Dinneny, \\ Pedro L. Rodriguez, Akira Nagatani, Yoichiroh Hosokawa, \\ Tobias I. Baskin, Tony P. Pridmore, Lieven De Veylder, \\ Hideyuki Takahashi \& Malcolm J. Bennett
}

20th March 2017

\section{Overview}

The image analysis methods developed to provide the data reported here comprises two processing pipelines:

1. An optic flow method matches local image patches between images to recover apparent motion. This produces a vector field; each pixel location is associated with a vector describing the image motion measured at that point.

2. Segmentation and contour analysis methods are applied independently to each image to identify the root centreline.

Motion vectors lying along the root centreline are then identified and may be analysed to provide growth estimates.

\section{Estimating Optical flow}

Optical flow algorithms estimate the motion of the viewed object(s) between consecutive image frames, often using differential methods. Various state of the art techniques [2, 17, 8, 12, 3] have been developed to improve this estimation since the pioneering work of Horn and Schunck [5]. 
Recently, a local descriptor matching component [4] has been incorporated to increase performance. Local image descriptors are extracted and matched from one image to the next. Local descriptors are sparse, scale invariant and extracted from rigid image regions (those which appear to simply translate, without any deformation, across the image plane). Matching is then generally reduced to a nearest-neighbour problem [13]. When traditional optical flow methods are extended in this way, the accuracy of the motion estimation increases significantly. False and ambiguous matches can, however, be reported when the descriptors are extracted from non-rigid regions. Correct matches can also be missed in weakly to moderately textured regions. The images considered here show root structures which are both non-rigid and textured.

To overcome this problem, the DeepFlow method [16] has been utilised here. In DeepFlow, a local descriptor is divided into 4 quadrants and matching is achieved by combining the result of matching quadrants independently. The approach explicitly assumes that each quadrant can move independently to some extent. When applied recursively, this strategy allows for fine, non-rigid matching with explicit pixel-wise correspondences. The pyramid response maps in the DeepFlow method are calculated via the bottom up process for every patch of the reference image to the target image. Local maxima in the response maps correspond to good matches of corresponding local image patches. Detail of its architecture was described in [16].

Figure 1 shows the optical flow result obtained from Frames 176 and 177 of one video sequence of the growing root using our implementation of DeepFlow, adapted from the initial code of the DeepFlow's authors. Apparent movements outside the root are the result of background noise.

\section{Estimating the Root Midline}

Given an image, a number of steps are required to estimate the midline of the root: separate the root from its background, remove root hairs and noise, calculate the skeleton and find the longest path through the skeleton.

\subsection{Extracting the root}

Level set based curve evolution [6] is a powerful method and is applied in many domains, including the analysis of biomedical images. The basic idea of the level set method is to represent a curve being fitted to some data implicitly, as the zero level set of a function defined over a regular grid. This function is then evolved (deformed) according to a specified speed function, inducing a related deformation in the curve. The speed function therefore controls fitting of the curve to the data. It is common for this function to contain terms controlling the relationship between the curve and the data points, and another constraining the overall shape, or smoothness, of the curve. Y. Shi and W.C. Karl in [10] proposed a fast two cycle algorithm for the approximation of level 
set based curve evolution, one cycle for the data dependent term and the second cycle for the smoothness regularisation.

One of the strengths of the level set method is that it can be generalised to an arbitrary number of dimensions. Here it is used to grow a region - the background component of a root image - rather than a curve. The user initialises the method by marking a rectangular area of the background. A level set method then extends this region, its speed function preventing the evolving level set from crossing the object boundaries - points at which the local image gradient is high. The effect is that the level set expands to cover the background region, but does not cross over into the root.

Figure 2 shows a sample root segmentation. Some parts of our implementation are adapted from the work of [14], particularly from [1].

\subsection{Removing root hairs and noise}

Since the intensity of the edge of the root hairs and the main root body are similar, especially when the root is mature, level set evolution will also stop at the boundaries of root hairs, as shown in Figure 2(b) and Figure 2(c), Water bubbles on the background can also be detected. These must be removed, and root hairs reduced, as far as possible.

First, all non-background objects are detected by creating a binary image in which background pixels are labelled 0 and non-background 1, and then extracting connected components. The largest non-background object is considered to be the root, and all other components are discarded (Figure 3(a)) The "rolling ball" algorithm, using a flat disk-shaped structuring element, then erodes the body of the root, removing, or at least reducing, root hairs. The algorithm is described in [15].

Finally, the new boundary of the root should be smoothed. The opening operation [15] is applied to reduce the noise produced by the trimming process and prepare for the next step, skeletonisation.

\subsection{Skeletonisation}

The skeleton of an object is the set of points that lie within that object at the (locally) maximum distance from the object boundary. The skeleton is useful in general because it provides a simple and compact representation of a shape that preserves many of its topological and size characteristics. It is of particular interest here because the root centreline must coincide with it. Many skeletonisation, or thinning, algorithms exist. The medial axis transformation (MAT) [11] is perhaps the most commonly used.

The approach taken here follows a common, two stage process. First, the MAT is applied to create an initial skeleton. Given a perfectly smooth-boundaried root object, the MAT will produce a single skeleton path that marks the centreline. In practise noise, in the form of small concavities and convexities on the root boundary, will generate spurious skeleton components linking the centreline to the boundary. Removal of these artefacts needs care, as it is easy to 
discard the true centreline. Short spurs (whose length is below a user-defined threshold) are first removed, then the longest path through the skeleton is sought.

\subsection{Finding the longest path}

Given the skeleton in the previous step, the midline of the root is defined as the longest path linking two end nodes. This is identified as follows:

1. Find all the end nodes, these are skeleton pixels which are adjacent to only one other skeleton pixel. Examples of detected end nodes are shown in Figure 5(a)

2. The user provides a starting point near the end of the root. The algorithm automatically finds the nearest end point and set it as the true starting point $S$.

3. Calculate the distance through the skeleton between the starting point $S$ and the remaining end points using quasi-euclidean distance [7] and retain the longest path. Figure 5(b) shows the midline overlaid on the root image.

In most cases, only two end nodes will exist and the longest path can be calculated quickly. However, depending on the structure and maturity of the root, our methods may not remove all root hairs. There may then be more than one branch in the skeleton, as shown in Figure 6(a) It has three end nodes and

one branch node. A branch node is defined as a pixel which has connections with at least three neighbour pixels. As shown in Figure 6(c), the longest path approach finds the correct midline despite this.

Note that, source codes and software for the above methods are available at https://github.com/tuanthng/RootAnalysis (in the Release tag).

\section{References}

[1] Fabien Bessy. Open, fast and efficient level set implementation, 2011.

[2] Michael J. Black and P. Anandan. The robust estimation of multiple motions: Parametric and piecewise-smooth flow fields. Computer Vision and Image Understanding, 63(1):75 - 104, 1996.

[3] Thomas Brox, Andrés Bruhn, Nils Papenberg, and Joachim Weickert. Computer Vision - ECCV 2004: 8th European Conference on Computer Vision, Prague, Czech Republic, May 11-14, 2004. Proceedings, Part IV, chapter High Accuracy Optical Flow Estimation Based on a Theory for Warping, pages 25-36. Springer Berlin Heidelberg, Berlin, Heidelberg, 2004. 
[4] Thomas Brox and Jitendra Malik. Large displacement optical flow: Descriptor matching in variational motion estimation. IEEE Transactions on Pattern Analysis and Machine Intelligence, 33(3):500-513, 2011.

[5] Berthold K. P. Horn and Brian G. Schunck. Determining optical flow. Artificial Intelligence, 17:185-203, 1981.

[6] Stanley Osher and James A. Sethian. Fronts propagating with curvaturedependent speed: Algorithms based on hamilton-jacobi formulations. J. Comput. Phys., 79(1):12-49, November 1988.

[7] David W Paglieroni. Distance transforms: Properties and machine vision applications. CVGIP: Graphical Models and Image Processing, 54(1):56 - 74, 1992.

[8] Nils Papenberg, Andrés Bruhn, Thomas Brox, Stephan Didas, and Joachim Weickert. Highly accurate optic flow computation with theoretically justified warping. International Journal of Computer Vision, 67(2):141158, 2006.

[9] S. Perreault and P. Hebert. Median filtering in constant time. IEEE Transactions on Image Processing, 16(9):2389-2394, Sept 2007.

[10] Y. Shi and W. C. Karl. A real-time algorithm for the approximation of level-set-based curve evolution. IEEE Transactions on Image Processing, 17(5):645-656, May 2008.

[11] Steven S. Skiena. The Algorithm Design Manual. Springer Publishing Company, Incorporated, 2nd edition, 2008.

[12] Deqing Sun, Stefan Roth, and Michael J. Black. Secrets of optical flow estimation and their principles, 2010.

[13] Richard Szeliski. Computer Vision: Algorithms and Applications (Texts in Computer Science). Springer, 2011 edition, October 2010.

[14] L. Şuta, F. Bessy, C. Veja, and M. F. Vaida. Active contours: Application to plant recognition. In Intelligent Computer Communication and Processing (ICCP), 2012 IEEE International Conference on, pages 181-187, Aug 2012.

[15] Rein van den Boomgaard and Richard van Balen. Methods for fast morphological image transforms using bitmapped binary images. CVGIP: Graphical Models and Image Processing, 54(3):252 - 258, 1992.

[16] P. Weinzaepfel, J. Revaud, Z. Harchaoui, and C. Schmid. Deepflow: Large displacement optical flow with deep matching. In 2013 IEEE International Conference on Computer Vision, pages 1385-1392, Dec 2013. 
[17] Manuel Werlberger, Werner Trobin, Thomas Pock, Andreas Wedel, Daniel Cremers, and Horst Bischof. Anisotropic huber-11 optical flow. In Proceedings of the British Machine Vision Conference (BMVC), London, UK, September 2009. to appear. 


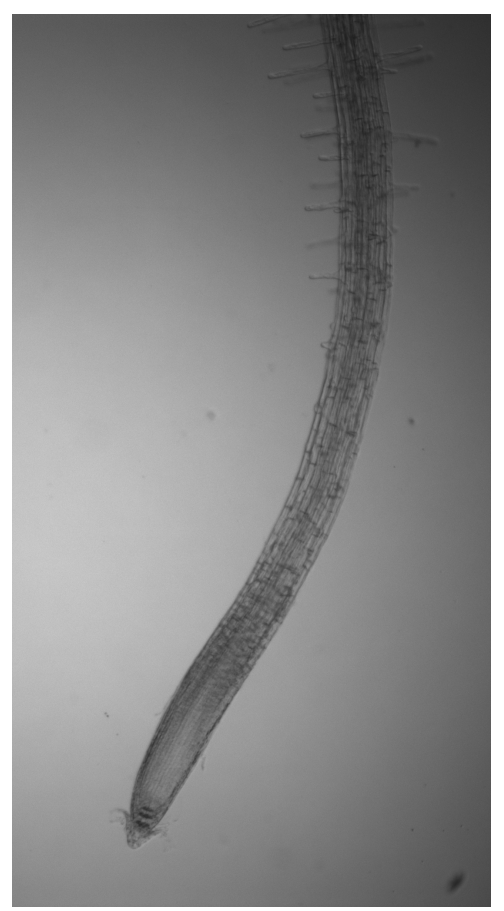

(a) Original Frame 176

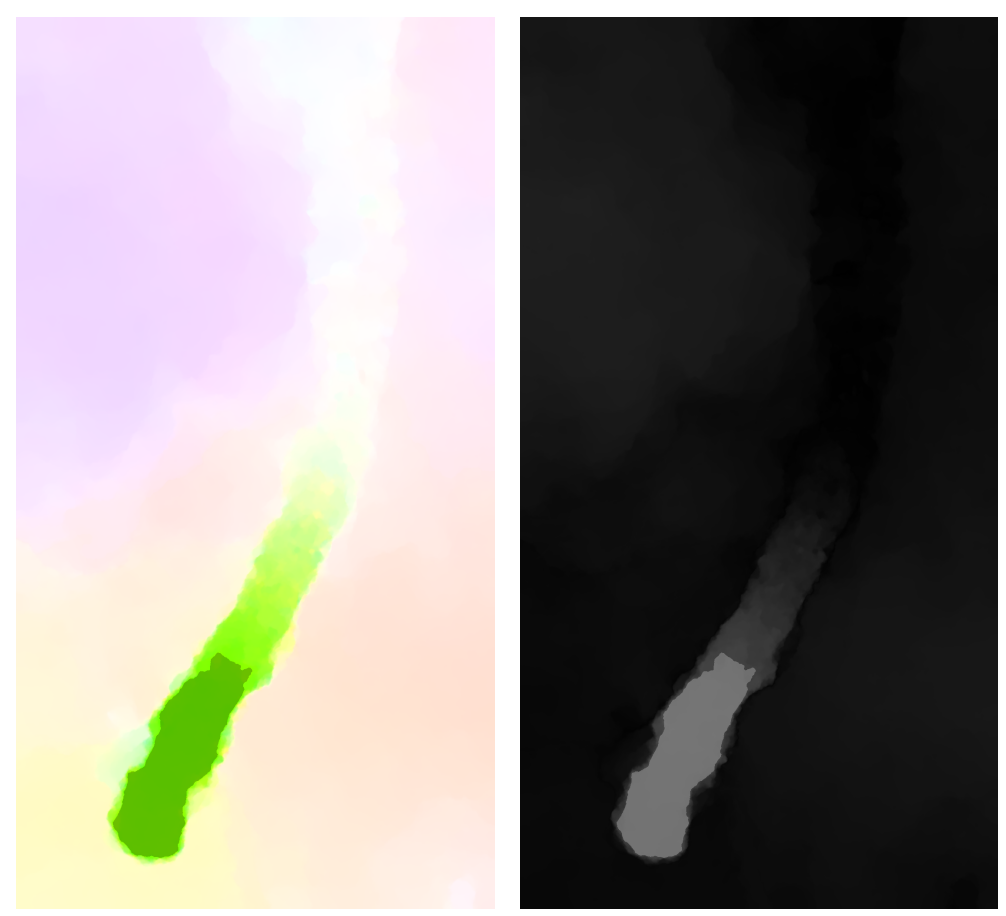

(c) Coloured Optical Flow between (d) Grayed Optical Flow between Frame Frame 176 and 177 Frames. The dark 176 and 177 Frames. The brightest region green region has a maximum velocity. thas a maximum velocity.

Figure 1: Examples of the optical flow result between two consecutive frames 176 and 177 of one growing root dataset. 

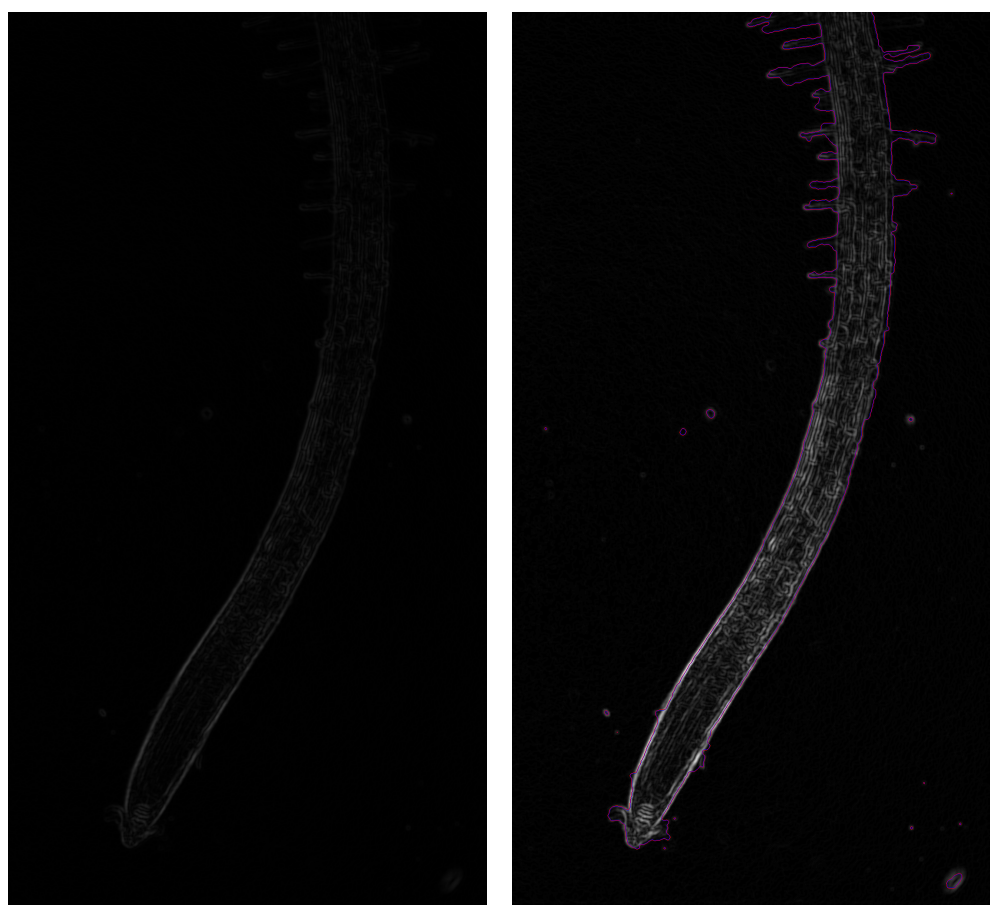

(a) Edge is detected in the Frame 176 and (b) Segmenting the root. The red line is smoothed by the median filter [9] covering the root boundary and noise.

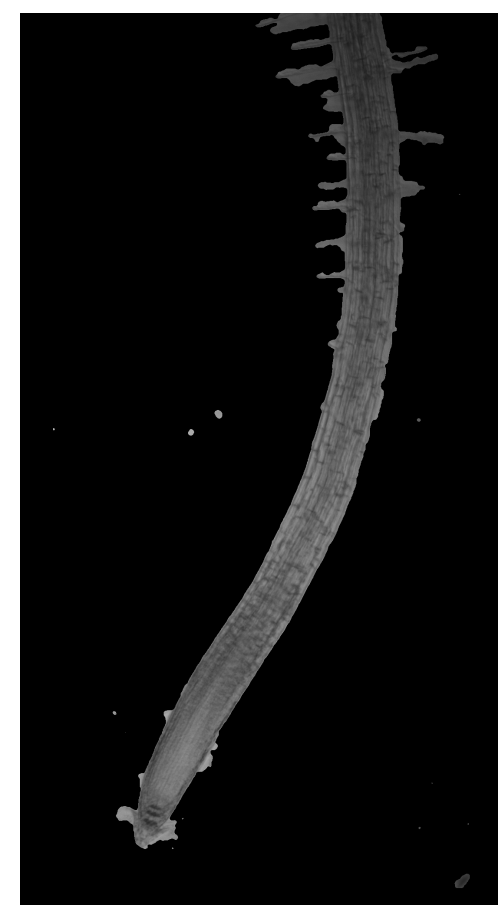

(c) The final root segmented in the Frame 176 after overdaying it on the original frame.

Figure 2: Examples of the root segmented in the Frame 176 of one growing root dataset. 

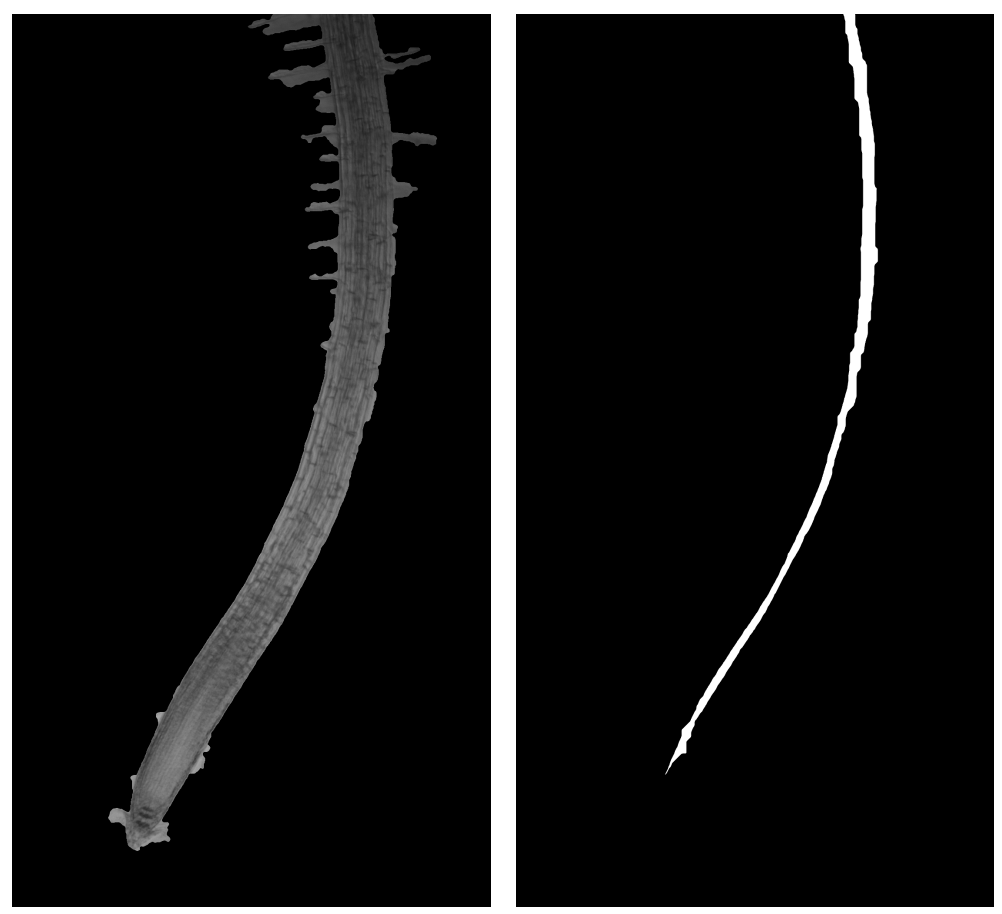

(a) The root in the Frame 176 after re- (b) The remain part of the root after removing all noises from the background. moving root hair.

Figure 3: Examples of the root hairs removed in the Frame 176 of one growing root dataset. 


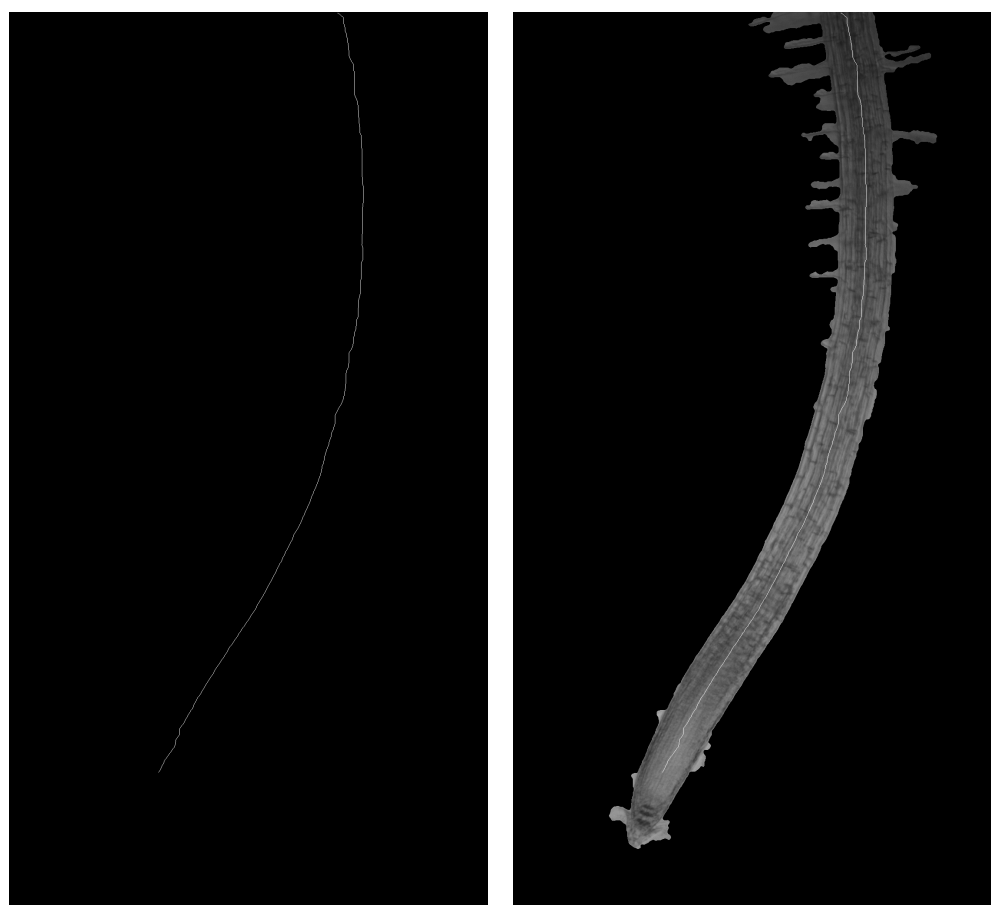

(a) The skeleton of the root of the Frame (b) The skeleton is overlayed on the root 176.

image (Figure $3(\mathrm{a})$.

Figure 4: Examples of the skeleton detected from the Frame 176 of one growing root dataset. 


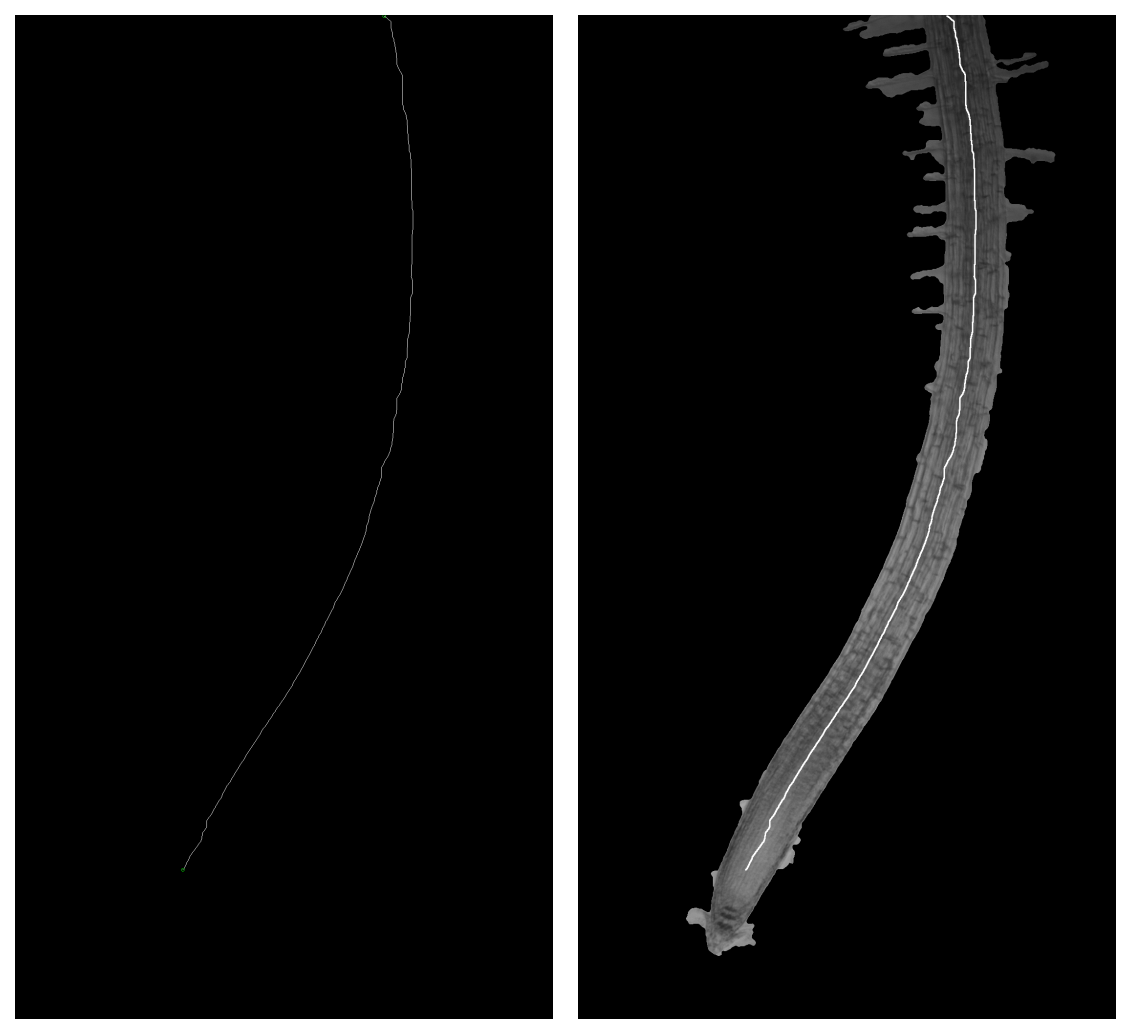

(a) The end nodes hightlighted in green $\operatorname{dot}(\mathrm{b})$ The midline hightlighted is overlayed on of the root of the Frame 176. the root image (Figure $3(\mathrm{a})$.

Figure 5: Examples of the midline detected from the skeleton of the Frame 176 of one growing root dataset. 


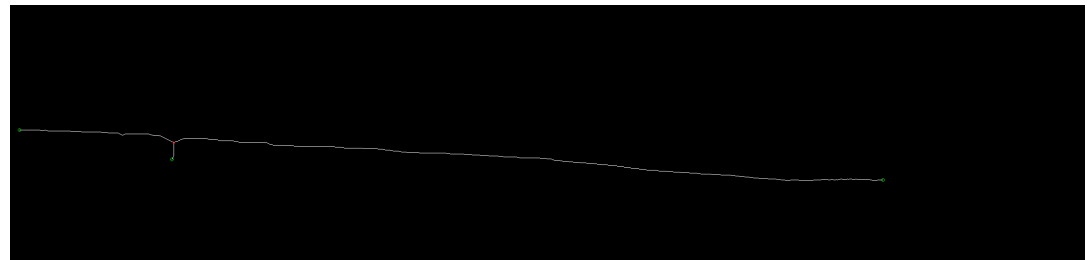

(a) In the Frame 126 of the second dataset, the end nodes are hightlighted in green dots and the branch nodes hightlighted in red dots.

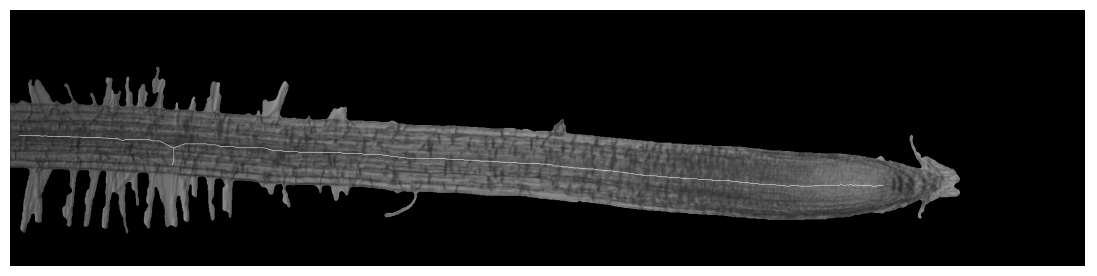

(b) The skeleton is overlayed on the root image.

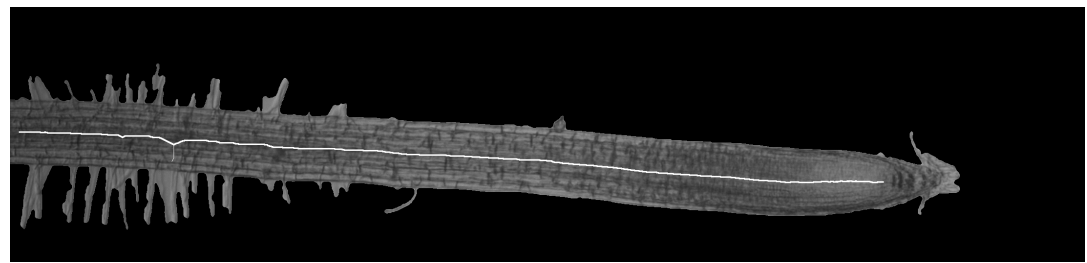

(c) The midline hightlighted is overlayed on the root image.

Figure 6: Examples of the midline detected from the skeleton of the Frame 126 of the second growing root dataset. 